\title{
Final Report on OPTIMASS: Optimizing Mass Storage Organization and Acces for Multi-Dimensional Scientific Data
}

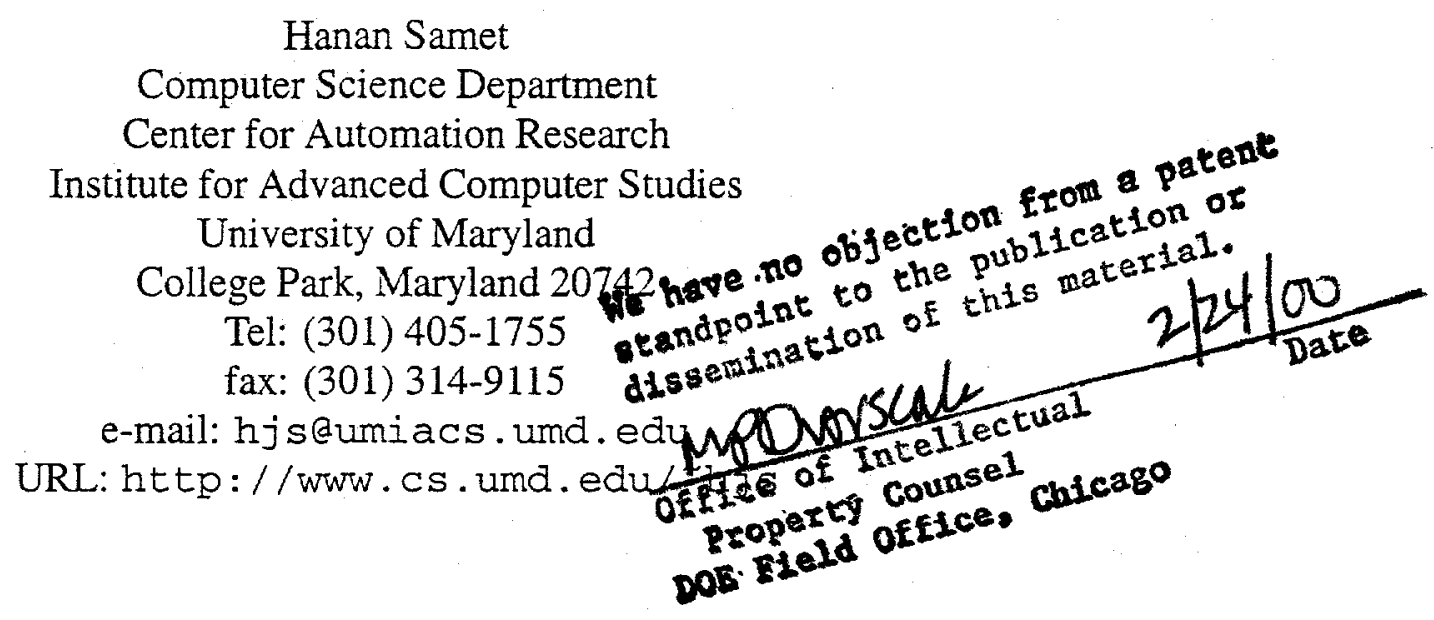

\section{Report Background and Organization}

This is a final report on the OPTIMASS project which was in part a collaborative research effort between researchers at the Lawrence Berkeley Laboratory, the Lawrence Livermore Laboratory, and the University of Maryland. The collaboration existed in the first two years of the project and the results of this work were reported in the appropriate progress reports. The formal collaboration was halted in 1997 as the Lawrene Lab groups were no longer funded to work on the project. This resulted in a refocusing of the effort of the Univeristy of Maryland group. During this time we shifted our attention to develop techniques to incorporate the data furnished to us into an existing spatial database known as Sand. This system was primarily designed for two-dimensional data and our work consisted of extending it to handle three-dimensional data. This activity is described in the resto of this report. Other work included the development of a nubmer of algorithms for dealing with spatial data and copies of some relevant papers and technical reports are included in the Appendix.

\section{Introduction}

This project investigated using the Sand technology to work with three-dimensional meteorological data. The specific data set we had represented the surface of the world as a 320 by 160 grid above which 19 height levels existed. Measurements, such as air temperature, were stored for across many time intervals. This data was stored in DRS (denoting Data Retrieval and Storage System) format (for which there was documentation, libraries of conversion functions, and conversion utilities). Instead of working directly with this fourdimensional array of measurements, Sand was converted to work with files representing an isosurface extracted from a particular time step and a particular measurement type (such as air temperature).

Sand previously was a GIS that allowed queries about country boundaries, river paths, and city locations. Often GIS queries involve objects of different type, such as what is the closest power plant to the border of a particular country. By adding the new object type 'isosurface', the system could handle queries such as what 


\section{DISCLAIMER}

This report was prepared as an account of work sponsored by an agency of the United States Government. Neither the United States Government nor any agency thereof, nor any of their employees, make any warranty, express or implied, or assumes any legal liability or responsibility for the accuracy, completeness, or usefulness of any information, apparatus, product, or process disclosed, or represents that its use would not infringe privately owned rights. Reference herein to any specific commercial product, process, or service by trade name, trademark, manufacturer, or otherwise does not necessarily constitute or imply its endorsement, recommendation, or favoring by the United States Government or any agency thereof. The views and opinions of authors expressed herein do not necessarily state or reflect those of the United States Government or any agency thereof. 


\section{DISCLAIMER}

Portions of this document may be illegible in electronic image products. Images are produced from the best available original document. 
is the closest city to a 270 -degree Kelvin temperature reading at a level greater than 2 (which, in conjunction with other measurements might indicate the potential for snow).

A major aspect of Sand was the notion of not just finding the closest city to a particular location or spatial object, but, instead, incrementally producing a list of all cities ordered by their distance from the particular location or spatial object. Thus, for example, if one was looking for a major manufacturing city near the Canadian border, one could launch a query for cities ordered by distance from the Canadian border and then inspect the list in order until a city occurred that 'seemed like' a major manufacturing center. This allows the user to be involved in the data analysis. An altemative approach would be for the user to have to quantify what is meant by 'major manufacturing center' and spend time 'debugging' the formal definition of such a subjective term, which would probably take longer than just scanning the list and intuitively classifying the cities in terms of their manufacturing capabilities. Another way of thinking about this is that Sand aids in the exploration of the data set. Presumably such capabilities would be useful to scientists exploring meteorological data as well.

In addition to aiding exploration of the data set by queries on spatial attributes, Sand also permits queries regarding non-spatial attributes. Taking our manufacturing center example a bit further, one might have stored with each city the amount of freight shipped from the city in a year. Rather than just asking for cities near the Canadian border, the user could ask for cities near the Canadian border that ship more than a certain amount of freight each year and thus have a smaller and more relevant list of cities to inspect.

In Sand, countries, rivers, and cities are not predefined types. Instead, the predefined types are their corresponding spatial objects such as polygons, sequences of line segments, and points are predefined types. A collection of cities then becomes a collection of points where the user knows that that particular collection of points corresponds to actual cities. Thus, at a more abstract level, one can view the original version of Sand as a database engine for working with two-dimensional geometry. The extensions necessary to handle isosurface data essentially mean extending Sand to handle three-dimensional geometry (i.e., points, line sequences, and triangular networks located in a three-dimensional space).

\section{Overview}

A version of Sand, called Sand3d, was created to investigate extending the Sand interface to handle threedimensional data. This work was based on an effort carried out in the first two years of the contract. It included figuring out how to get itcl (the underlying interpreter for the Sand interface) to talk to OpenGL (a standard graphics library that supports three-dimensional display primitives). It also included figuring out how to convert the DRS data files into isosurfaces represented as polygonal data. This was done using the Visualization Toolkit [3] in combination with the DRS software that came with the initial raw data. A script was written in Nawk that converted the polygonal isosurface data into a triangular strip format that Sand3d work with directly. It is frequently convenient to represent three-dimensional surfaces as networks of triangles. A compact way of storing such networks is 'triangular strip format', which decomposes the network into linear sequences of triangles that share common edges (so that the edge only needs to be stored once rather than having to be stored separately with each of the two triangles that share it).

The overall structure of Sand is a collection of routines written in $\mathrm{C} / \mathrm{C}++$ for manipulating spatial indices connected to an interface written in iTcl (an object-oriented version of $\mathrm{Tcl}$ ). Extensions to iTcl to allow it to interact with a window running OpenGL (initially the public Mesa version of OpenGL, but later also the faster OpenGL version distributed with Sun's SOLARIS operating system) already existed. So the main focus of the work was in creating a collection of functions in the $\mathrm{C} / \mathrm{C}++$ spatial index library of Sand and then making what modifications were necessary in order to the iTcl interface to invoke these $\mathrm{C} / \mathrm{C}++$ functions properly. 


\section{Scroll Bars}

One issue that arose in extending the original Sand interface was determining what the three-dimensional analogs of the scroll bars would be. The basic idea behind scroll-bars is that we are specifying a location within a range - the extremes of the range being represented by the extreme positions of the scrollbar and other positions on the scroll-bar are viewed as linearly interpolated onto the value being controlled (such as the horizontal or vertical location of the center of the screen). In the two-dimensional case, it is rather straightforward to talk about a bounding rectangle around the map data and then scrolling a window over that bounding rectangle. In the case of three-dimensional data, the bounding corresponding bounding rectangle would change with the viewing angle and would be more difficult to calculate. Thus the scrollbars were removed from the interface.

In OpenGL, a general model of a viewpoint and a viewing frustum are implemented. $\mathrm{C} / \mathrm{C}++$ code was written to give iTcl access to this functionality. However, the current iTcl code does not implement arbitrary camera motion, but instead uses a fixed predefined frustum description and handles zooming by moving the viewpoint closer to the object being viewed. Rotating the map along its $\mathrm{x}$-axis (or $\mathrm{y}$-axis or $\mathrm{z}$-axis) is also supported. However, moving the map to the left or right (or up or down) is not currently supported in the user interface.

Some of these features are controlled by global variables in the $\mathrm{C}$ code. Since these globals are normally invisible to the iTcl code, special $\mathrm{C}$ functions for reading and setting these values were implemented to give the iTcl code access to these variables.

A related problem is whether to perform parallel or perspective transformation of the three-dimensional data. The actual transformations are handled by OpenGL and so either implementation is equally easy. However, we found that parallel projections tended to be easier to interpret (when the viewpoint is close to the object being viewed, perspective can be less intuitive).

\section{Highlighting}

Another problem was dealing with highlighting. In the original Sand, a red box was drawn around the object selected. In the three-dimensional case, such a box would again have to vary with the viewing angle. There was also an underlying problem with the way Sand approached drawing in that by drawing one object in twodimensions later than another, the second object would end up obscuring the other (even though it had the same 'height'). When true three-dimensional coordinates were used with a generic three-dimensional rendering package (OpenGL), the order in which objects were 'drawn' no longer determined visibility. To get the same effect, it was necessary to draw the 'later' object slightly closer to the viewer than the original object.

\section{New Classes}

Classes were implemented for the data types: Point3d, Line3d, Box3d, Triangle3d, and Trianglestrip3d. Point $3 d$ and Line3d are three-dimensional extensions of the original two-dimensional Point and Line classes. Box $3 \mathrm{~d}$ is the three-dimensional version of Rectangle. Box $3 d$ is not a drawing primitive, like Point $3 d$ and Line $3 d$, but rather is used to represent the portion of space controlled by a node in the disk-based arbitrary-dimensional PMR quadtree that underlies the Sand spatial index system. 
Triangle $3 \mathrm{~d}$ is the basic building block for three-dimensional surfaces. It can be viewed as an extension of the two-dimensional polygon class. However, triangles are restricted to having only three sides. Converting arbitrary polygons to triangles was not implemented. It would also be useful to allow Triangle3d to specify which edges are to be visible and which invisible (to allow a square to be represented by two triangles without having to draw a diagonal line through the square). Although triangles work well with the volume data, more work is needed to have them interface well with the earlier two-dimensional map data (that relied heavily on concave polygons with many sides to describe country boundaries).

Triangles have many advantages over three-dimensional general polygons. A major problem when working with general polygons in three-dimensional space is in keeping all the vertices of the polygons coplanar. Since triangles have only three vertices, they are automatically coplanar. Another problem with general polygons is that OpenGL doesn't support three-dimensional concave polygons (i.e., it only supports threedimensional convex polygons). The same efficiency concerns that caused the OpenGL designers to not support this feature would also cause difficulties for any application that tried to support general three-dimensional polygons.

Trianglestrip3d is a compact way to manipulate a collection of triangles that correspond to a path across a surface. Programs such as the Visualization Toolkit often produce this sort of data when converting volume representations to isosurface representations. Also display programs, such as OpenGL, have builtin support for displaying this sort of connected sequence of triangles. Although the current version of Sand3d doesn't take advantage of this feature of OpenGL, a later version could easily do so to achieve faster display rates.

These classes were added to the file Geometry.cc . Specifically, the additions included:

1. A new enumerated type Direction $3 d$ defining the 26 possible directions from a node to a neighbor in a three-dimensional grid. The original Direction type defined the 8 possible directions from a node to a neighbor in a two-dimensional grid, i.e., NW (north west), N, NE, W, E, SW, S, and SE. To the North/South and East/West polarities of the two-dimensional grid, the three-dimensional grid adds Backward/Forward. Point3d_type:

(a) Constructors that build a $3 \mathrm{~d}$ point from a $2 \mathrm{~d}$ point, another $3 \mathrm{~d}$ point, or from 3 coordinate values.

(b) == and ! = comparison operators to test if two points are the same (have the same coordinates) or not.

(c) + and - define vector arithmetic on the point type.

(d) IsCompatible checks to see if an object is of Point3d_type.

(e) Assign assigns the coordinate values of one point to another.

(f) ComparePoint 3 ds returns 0 if Float: : CompareFloats on the corresponding coordinates of two points are 0 , otherwise returns the first non-zero value returned by CompareFloats.

(g) Compare casts an object to a Point3d_type and then uses ComparePoint3d to compare it with another Point3d_type. If the object is not of Point3d_type, then it returns -2 .

(h) GetAttr takes a Point $3 \mathrm{~d}$ and an index and returns the corresponding coordinate value in the point.

(i) Scan sets the values of a Point $3 d$ to those stored textually in a AttrData arg.

(j) Format builds the AttrData arg that corresponds to a Point $3 d$ object. Format and Scan together allow class objects to be stored in files and read back later. 
(k) A similar pair of methods, Load and BinFormat are also implemented, allowing an alternative more compact data storage format for the class.

2. Line3d_type

(a) Constructors for a $3 \mathrm{~d}$ line from another $3 \mathrm{~d}$ line, from a $2 \mathrm{~d}$ line, from two $3 \mathrm{~d}$ points, or from six coordinates.

(b) The ==, !=, IsCompatible, Assign, Compare, GetAttr, Scan, Format, Load, and Binformat methods are also defined for this class.

3. Box3d_type

(a) Constructors from another $3 \mathrm{~d}$ box, a $2 \mathrm{~d}$ rectangle, two $3 \mathrm{~d}$ points (lowerlefthand corner and the other corresponding to the dimensions (width, height, and depth) of the box), six coordinate values again corresponding to the lowerlefthand corner and dimensions of the box and from a $3 \mathrm{~d}$ line (resulting in the bounding box for that line segment). All boxes are rectilinear (i.e., aligned with the $\mathrm{x}, \mathrm{y}$, and $z$ axes).

(b) FNWcorner, ENEcorner, FSWcorner, FSEcorner, BNWcorner, BNEcorner, BSWcorner, BSEcorner, Nside, Sside, Wside, and Eside to access various corners and sides of the box.

(c) The $==$, ! =, IsCompatible, Assign, Compare, GetAttr, Scan, Format, Load, and Binformat methods are also defined for this class.

4. Triangle3d_type

(a) Constructors from another $3 \mathrm{~d}$ triangle, three $3 \mathrm{~d}$ points, or nine coordinate values.

(b) first_corner, second_corner, third_corner, first_side, second_side, and third_side methods give access to the points that define the triangle and the sides of the triangle.

(c) The ==, !=, IsCompatible, Assign, Compare, GetAttr, Scan, Format, Load, and Binformat methods are also defined for this class.

5. Trianglestrip3d_type

(a) compute_bounds computes the bounding box for a collection of $3 \mathrm{~d}$ points.

(b) Constructor from a polygon (currently just a stub returning not_implemented), from another $3 \mathrm{~d}$ triangle strip, and from a sequence of $3 \mathrm{~d}$ points (corresponding to the vertices of a $3 \mathrm{~d}$ triangle strip).

(c) FirstCorner, NextCorner, PrevCorner, LastCorner, and ThisCorner for accessing the list of vertices in a $3 \mathrm{~d}$ triangle strip.

(d) NumberofTriangles returns the number of triangles in the triangle strip.

(e) FirstTriangle and NextTriangle give access to the $3 \mathrm{~d}$ triangle strip as if it were a sequence of triangles.

(f) EnclosingBox returns the bounding box for a 3d triangle strip.

(g) [ ] allows one to access the vertices of a triangle strip using an array operator.

(h) = allows one to assign one triangle strip to another.

(i) The ==, !=, IsCompatible, Assign, Compare, GetAttr, Scan, Format, Load, and Binformat methods are also defined for this class. 
(j) Special constructor and destructor had to be written for triangle strips to manage the array of vertices.

(k) non_self_intersecting tests to see if a triangle strip intersects itself.

In addition to these classes, some free-standing polymorphic functions had to be defined. In particular, they are:

1. classify that returns the relative position (as a Direction $3 \mathrm{~d}$ ) of a $3 \mathrm{~d}$ point with respect to a $3 \mathrm{~d}$ box.

2. determinant $3 \mathrm{~d}$ returns the determinant of a $3 \mathrm{~d}$ point following the conventions of A Programmer's Geometry $[1]$ - this reference was frequently useful.

3. length $3 d$ returns the distance of a point from the origin.

4. classify for two $3 \mathrm{~d}$ lines returns whether they are parallel, intersecting, or neither.

5. classify a point with respect to a line segment returns whether, when the point is projected onto the line of the line segment, does it fall within the bounds of the two points of the line segment or, if not, then on which side of the line segment does it fall.

6. DistancePoint3apoint3d returns the distance between two 3d points. DistancePoint3dPoint and DistancePointPoint $3 \mathrm{~d}$ coerces the $2 \mathrm{~d}$ point into a $3 \mathrm{~d}$ point and then performs DistancePoint3dPoint3d.

7. DistancePoint $3 \mathrm{dLine} 3 \mathrm{~d}$ returns the distance between a $3 \mathrm{~d}$ point and a $3 \mathrm{~d}$ line. Again, the corresponding $D i s t a n c e P o i n t L i n e 3 d$ and DistancePoint $3 d L$ ine are defined.

8. DistancePoint $3 \mathrm{~dB} 0 \times 3 \mathrm{~d}$ returns the distance from a $3 \mathrm{~d}$ point to a $3 \mathrm{~d}$ box. Again the corresponding DistancePointBox3d and DistancePoint 3 dRect are defined.

9. DistancePoint3dTriangle3d is defined using the helping functions DistancePoint3d-

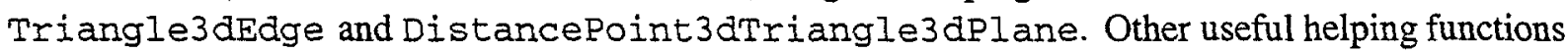
are InsidePoint 3 dTriangle $3 \mathrm{~d}$ and OverPoint 3 dTriangle $3 \mathrm{~d}$.

10. IntersectLine $3 d \mathrm{dTr}^{2}$ angle $3 \mathrm{~d}$ indicates if a $3 \mathrm{~d}$ line intersects a $3 \mathrm{~d}$ triangle.

11. DistancePoint3dTriangleStrip3d (and the corresponding DistancePointTriangleStrip3d) return the distance from a point to a triangle strip.

12. DistancePoint3dPoly is implemented, but makes use of the unimplemented conversion of a polygon into a $3 \mathrm{~d}$ triangle strip.

13. Distanceline3aLine3d and the corresponding Distancelineline3d and DistanceIine 3 di ine return the distance between two line segments.

14. Distanceline3driangle $3 \mathrm{~d}$ returns the distance between a $3 \mathrm{~d}$ line and a triangle.

15. DistanceTriangle3dTriangle3d returns the distance between two triangles.

16. Distanceline3dBox3d and the corresponding functions DistancelineBox3d and Distanceline 3 dRect return the distance between a line and a box. 
17. DistanceTriangle $3 \mathrm{dBO} \times \mathrm{d} \mathrm{d}$ returns the distance between a triangle and a box.

18. DistanceLine3dTrianglestrip3d returns the distance between a line and a triangle strip.

19. DistanceBox3dBox3d and the corresponding DistanceRectBox3d and DistanceBox3dRect) return the distance between two boxes.

20. DistanceBox3dTrianglestrip3d returns the distance between a box and a triangle strip.

21. DistanceTrianglestrip3aTrianglestrip3d (computing the distance between two triangle strips) returns the distance between two triangle strips.

22. Another family of such functions that test for intersection rather than compute distances was also implemented.

23. Another family of such functions that test for containment (or whether one object is inside another) was also implemented. There is also a family of functions for non-containment (or whether one object is outside another) that were implemented.

24. Functions for computing the bounding box of a $3 \mathrm{~d}$ point, line, box, or triangle strip were implemented.

25. Functions for determining if a $3 \mathrm{~d}$ point or $3 \mathrm{~d}$ line are above a polygon were implemented.

While the amount of work one needs to do to add a new type class to Sand initially appears constant, it is actually linear in the number of other types that Sand supports, since in order for queries to be posed on attributes of different types, the pairwise functions Distance, Intersect, Inside, and Outside need to be implemented for every possible pair of types. Furthermore, since Sand works primarily with spatial attributes, these pairwise functions are invoked quite frequently by the system and thus benefit from efficient implementation. They must also be very reliable.

In addition to creating these functions and types, many changes had to be made in SandSpatiallndex.cc, SandSpatialJoin.cc, and SandTable.cc to take into account that the bounding boxes coming back from the arbitrary-dimensional PMR quadtree queries were three dimensional rather than two dimensional.

Generalizing all this work for $\mathrm{k}$-dimensional objects would allow Sand to have the capability to work with isosurfaces across time rather than having to deal with individual time slices as is now the case. Potentially this could also allow some flexibility between when deciding whether an attribute is considered as a 'spatial attribute' and when its is considered as a numeric 'non-spatial attribute'.

\section{Spatial Join}

A key feature of Sand is the focus on the spatial join as a major mode of interacting with the data. The spatial join code was extended to work with the new three-dimensional geometric classes described above.

A spatial join is a join where the join condition involves a spatial attribute. For example, consider a rivers relation (spatial attribute is the line segments) and a roads relation (spatial attribute is the line segments). In this case, a spatial join of rivers and roads where the join condition is "occupancy of the same locations" is the set of points common to both relations. In particular, the result of the join is the set of tuples (Cartesian product of them) where each tuple in the result is formed by a pair of tuples (one from roads and one from rivers) where the spatial attribute values have at least one point in common. Thus, in Sand, any time one wants 
to ask a question about how information in two different data sets relate, it is expressed as a join (or a semijoin, which is discussed in the next section).

Prior to the development of a three-dimensional version of Sand, there was a two-dimensional version. This two-dimensional version was developed using data sets that represented country boundaries as polygons, rivers as sequences of line segments, and cities and nuclear monitoring stations as points. Since two-dimensional line segments can be viewed as a special case of three-dimensional line segments and two-dimensional points can be viewed as a special case of three-dimensional points, joins can be performed between new three-dimensional data sets and previously existing two-dimensional data sets. The one current counter-example to this is the countries boundary data since, as noted in the previous section, methods were not implemented for computing the distance between three-dimensional triangles and arbitrary two-dimensional polygons.

The three-dimensional data set that the three-dimensional version of Sand was developed to explore was a set of isosurfaces extracted from meteorological data, specifically temperature measurements at various altitudes and locations at various times. One data set type of interest would consist of the isosurfaces from a particular time that corresponded to a particular temperature boundary. Note that it is not necessary that all the temperature readings with a particular value form a single connected region. Thus a particular temperature boundary may be the union of many surfaces. Another data set type of interest is one where all the isosurfaces from a particular time are in the same data set and each is labeled by its corresponding temperature value.

An example of a join operation involving three-dimensional data would then be to compute the join of the locations of the cities in the two-dimensional plane of the surface of the Earth with a collection of triangle strips corresponding to the isosurface for a particular temperature. The resulting tuples would be ordered (i.e., ranked by their separation or distance between them). This would allow us to find which cities are closest to a particular temperature isosurface. Notice that neither the isosurface nor the connected components of the isosurface form natural entities in the isosurface data set. Instead, the isosurface data set is the union of a collection of triangle strips. Thus, in the above join, each city would appear in as many tuples of the resulting set as there are triangle strips in the data set. However, since we are viewing the result of the join in the order of distance (i.e., a ranking), most of these pairings would never be seen.

A useful extension of this result would would be to first label all the triangle strips associated with a particular connected component of an isosurface with a particular value and then do the join operation against that label rather than the individual objects. These extensions are currently being investigated via the development of more complex iterating units. In particular, the iterating unit of the ranking would now be the actual isosurface rather than the individual triangles.

\section{Spatial Semijoin}

An important variation on the spatial join is the spatial semi-join. The spatial semijoin code was extended to work with the new three-dimensional geometric classes described above. Briefly, the spatial semijoin is motivated by the regular semijoin operation in relational databases. In essence, the semijoin of relations $A$ and $B$ with join condition $C$ is formed by initially taking the Cartesian product of sets $A$ and $B$ and then just retaining the tuple pairs where condition $C$ is satisfied. At this point, we have the conventional join. For example, the answer might be the set of tuples: $(a 1, b 2)(a 1, b 3)(a 2, b 4)(a 2, b 5)(a 3, b 6)$ where ai corresponds to the attributes from relation $\mathrm{A}$ and bi corresponds to the attributes from relation $\mathrm{B}$.

The difference between the semijoin and the join operation is that in the semijoin we also specify a surviving relation. The semijoin with relation $A$ as the survivor (like a projection on the attributes of relation $A$ ) means that after the join is computed, we only retain the attributes of the Cartesian product that came from relation $A$ 
(this is the projection). This means that in our example, the set of tuples $(a 1, b 2)(a 1, b 3)(a 2, b 4)(a 2, b 5)(a 3, b 6)$ would be replaced by (a1) (a1) (a2) (a2), (a3). Now, notice that there are two instances of a1 and a2 (i.e., the attribute values). So, the question remains which one do we keep? It does not really matter in the conventional definition of the semijoin. The same is true for the conventional definition of the spatial semijoin. The only important item to note is that the join condition in the spatial semijoin involves a spatial attribute.

In the above, we actually defined a "ranking spatial semijoin". In this case, we say that if there are several duplicate instances after taking the projection, then we retain the one with the smallest distance between the spatial attributes of the two joined relations. As an example, of its utility, suppose that we have one relation called stores and another called warehouses. In this case if we compute the ranking spatial semijoin of stores and warehouses with stores as the surviving relation, then we have the result that of the (store,warehouse) tuple pairs, we retain the (store, warehouse) pair with the smallest distance between the two objects in the pair. What we have done is use the ranking (i.e., distance value between the two spatial attribute values of the relation) to decide which of the many tuples with the same store to retain (just one of them).

As an example of the utility of the spatial semijoin (actually the ranking spatial semijoin, suppose that we compute a ranking spatial semijoin between a data set of cities and a dataset of all isosurfaces from a particular time ranked by distance. This would give us each city associated with its nearest isosurface (temperature reading). Issues relating to the efficient computation of the spatial join and ranking spatial semijoin are part of an ongoing research effort (e.g., [2]).

\section{References}

[1] A. Bowyer and J. Woodwark. A Programmer's Geometry. Butterworth's, Guilford, United Kingdom, 1983. (second edition 1988).

[2] G. R. Hjaltason and H. Samet. Incremental distance join algorithms for spatial databases. In Proceedings of the ACM SIGMOD Conference, pages 237-248, Seattle, WA, June 1998.

[3] W. Schroeder, K. Martin, and B. Lorensen. Visualization Toolkit. Prentice-Hall, Englewood Cliffs, NJ, 1996. (available at http://gd. tuwien.ac.at/visual/vtk/vtk-www/). 
Appendix - Relvant Publications Issued Under the Contract 


\title{
Incremental Distance Join Algorithms for Spatial Databases*
}

\author{
Gísli R. Hjaltason and Hanan Samet \\ Computer Science Department and \\ Center for Automation Research and \\ Institute for Advanced Computer Studies \\ University of Maryland \\ College Park, Maryland 20742 \\ grh@cs.umd.edu and hjs@cs.umd.edu
}

\begin{abstract}
Two new spatial join operations, distance join and distance semijoin, are introduced where the join output is ordered by the distance between the spatial attribute values of the joined tuples. Incremental algorithms are presented for computing these operations, which can be used in a pipelined fashion, thereby obviating the need to wait for their completion when only a few tuples are needed. The algorithms can be used with a large class of hierarchical spatial data structures and arbitrary spatial data types in any dimensions. In addition, any distance metric may be employed. A performance study using Rtrees shows that the incremental algorithms outperform non-incremental approaches by an order of magnitude if only a small part of the result is needed, while the penalty, if any, for the incremental processing is modest if the entire join result is required.
\end{abstract}

\section{Introduction}

The spatial join operation is similar to the join operation in relational databases. It is defined on two sets of objects, and computes a subset of the Cartesian product of the two sets, determined by a spatial predicate, which prescribes a certain spatial relationship between the objects in the result. The most common spatial predicate is intersect, i.e., the geometry of the objects are required to intersect $[1,7,8,19,21,22]$. A generalization of this is within, where the objects are required to lie within some distance of each other $[24,29]$. Other spatial predicates have been considered as well, and general methods to compute a spatial join proposed $[4,14]$. Some of these methods involve special join indexes $[14,24]$.

In this paper, we define a "distance join" operation, which computes a subset of the Cartesian product of sets $A$ and $B$, and speci-

"This work was supported in part by the National Science Foundation under Grant IRI-9712715 and the Department of Energy under Contract DEFG0295ER25237. fies an order on the result, based on distance. The distance is usually defined in terms of spatial attributes, but this need not be the case. When the distance of the resulting pairs is limited to a range, we have a generalization of a spatial join based on a within predicate. The "distance semi-join" is a useful special case of the distance join which for each object in $A$ finds the nearest object in $B$. Figure 1 defines the distance join and distance semi-join operations using a syntax loosely adapted from SQL-92, including the STOP AFTER clause extension proposed in [10]. The WHERE and STOP AFTER clauses, specifying limits on the distance and/or the number of result tuples, are optional. These basic queries could be made more complicated by adding further selection conditions in the WHERE clause.

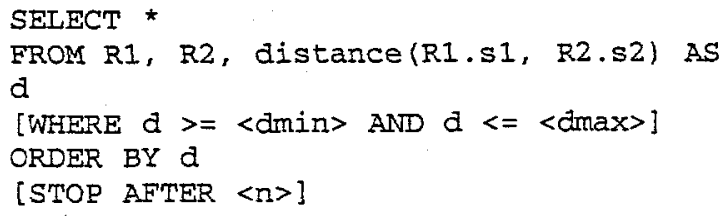

(b)

Figure 1: Definition of (a) distance join and (b) distance semijoin using SQL.

The distance join and distance semi-join have numerous useful applications in spatial databases. For example, given a spatial database of rivers and cities, we can use partial computation of them to "find the city nearest to any river", "find the city nearest to any river, such that the city has a population of more than 5 million", and "find cities within 5 miles of any river". The distance semi-join is useful as a clustering operation. For example, suppose we are given two relations consisting of the locations of stores and of warehouses, respectively, and for each store we wish to determine the closest warehouse. This is achievedby taking the distance semi-join of the stores relation with the warehouse relation. The distance semi-join works 
by reporting the (store,warehouse) pairs in order of distance. Note that once we have determined the closest warehouse to a particular store, that store does not participate in other tuples with the remaining warehouses.

Computing the complete distance semi-join yields a clustering of the stores. In fact, for point data, the result partitions the space in a manner analogous to a discrete Voronoi diagram, i.e., each point in the stores relation is associated with the closest point in the warehouse relation (thus, in the terminology of Voronoi diagrams, the locations of the warehouses are the sites). The attractiveness of this analogy lies in providing users a mechanism to perform a geometric operation such as the Voronoi diagram using a data base primitive without having to invoke a special purpose algorithm from a geometric library to perform the operation. Note that this operation is not symmetric. In particular, the result of computing the distance semi-join of the warehouse relation and the stores relation is that for each warehouse, we get the closest store.

The clustering join [32] is similar to the distance semi-join with the difference being that the clustering join is symmetric. An algorithm for computing the clustering join is also given in [32]. However, that algorithm is not well suited for spatial data that resides in $d$-dimensional Euclidean space. The reason is that [32] deals with more general objects-such as patterns, strings, trees, graphs, etc.-whose internal structure is unknown as far as the algorithm is concerned. The only knowledge about the objects comes from a distance measure that returns the distance between two objects. Furthermore, the distance measures are assumed to be expensive to compute, so that the overall goal is to compute as few distances as possible. In contrast, spatial data allows the use of spatial indexes which in effect summarize the data and enable avoiding many distance calculations (which, however, are not necessarily the most expensive component of query algorithms involving distances).

In this paper we present incremental algorithms for computing the distance join and distance semi-join in the sense that the pairs resulting from the corresponding operation are reported one-by-one. This enables a query engine to use the algorithms in a pipelined fashion. Furthermore, the algorithms aim to deliver results as soon as possible. Such "fast first" pipelined join methods have recently become a focus of attention $[3,33]$. They have become important in enabling the development of more user friendly and interactive interfaces to database systems [16]. Recent proposals for extending $S Q L[10]$ also benefit greatly from the presence of such algorithms.

A variation of our incremental distance join algorithm can be used to compute intersecting pairs [30], closest pair [6], and all nearest neighbors $[2,11,31]$ in a set of objects. While our incremental distance join algorithm may not always be competitive with some of the above algorithms in terms of computational complexity, it may nevertheless be a reasonable alternative given that a spatial data structure has already been built. In addition, unlike most of these methods, it is not limited to point or rectangle objects.

The rest of this paper is organized as follows. Section 2 describes the incremental algorithms for computing the distance join and distance semi-join. Section 3 describes the environment in which we perform our experiments, and Section 4 presents the results. Section 5 concludes with a number of future tasks.

\section{Incremental Distance Join Algorithms}

In this section we describe our incremental distance join algorithm. Although our algorithm is general in the sense that it can be used with most spatial data structures, for concreteness we present it in the context of the R-tree. Also, performance tests were conducted with R-trees (see Section 4). The rest of this Section is organized as follows. Section 2.1 reviews the R-tree. Section 2.2 describes the basic incremental algorithm for the distance join, followed by an outline of a number of methods for extending its functionality as well as improving its performance. Section 2.3 presents modifications to the basic algorithm to enable it to compute the distance semi-join operation.

\subsection{R-trees}

The R-tree [15] (see Figure 2) is one of many proposed spatial data structures. It is an object hierarchy in the form of a balanced structure inspired by the $\mathrm{B}^{+}$-tree [12]. Each $\mathrm{R}$-tree node contains an array of (key, pointer) entries where key is a hyper-rectangle that minimally bounds the data objects in the subtree pointed at by pointer. In an R-tree leaf node, the pointer is an object identifier (e.g., a tuple ID in a relational system), while in a non-leaf node it is a pointer to a child node on the next lower level. The maximum number of entries in each node is termed its node capacity or fan-out and may be different for leaf and non-leaf nodes. The node capacity is usually. chosen so that a node fills up one (or a small number of) disk pages. $\mathrm{R}$-rees can be used to index a space of arbitrary dimension and arbitrary spatial objects rather than just points.

As described above, $R$-tree leaf nodes contain a minimal bounding rectangle and an object identifier for each object in the node, i.e., the geometric description of the objects is stored external to the Rtree itself. Another possibility is to store the actual object, or only its geometric description, in the leaf instead of the bounding rectangle. This is usually only useful if the object representation is relatively small (e.g., similar in size to a bounding rectangle) and is fixed in length. If the entire object data (i.e., all relevant attributes) are stored in the leaf nodes, then the object identifiers need not be stored. The disadvantage of this approach is that objects will not have a fixed address, as some objects must be moved upon each R-tree node split.

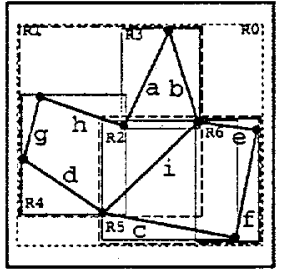

(a)

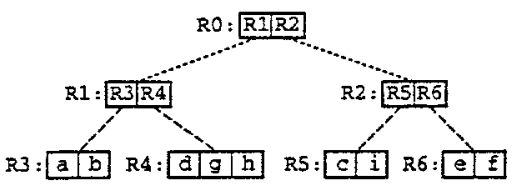

(b)
Figure 2: An R-tree for a set of 9 line segments. (a) Spatial rendering of the line segments and bounding rectangles, and (b) a tree access structure for (a). Bounding rectangles for individual line segments are omitted from (a) in the interest of clarity.

We make use of an $\mathrm{R}$-tree variant called the $\mathrm{R}^{*}$-tree [5]. It differs from the conventional $R$-tree in employing a more sophisticated insertion and node-splitting algorithms that attempt to minimize a combination of overlap and area increase between minimum bounding rectangles.

\subsection{Computing Distance Join}

Our incremental distance join algorithm may be viewed as simultaneously applying an incremental nearest neighbor algorithm [18] (see [17] for the application of a similar approach to the LSD tree) to the two spatial data structures corresponding to the spatial attributes of the joined relations. The algorithm works for any spatial data structure based on a hierarchical decomposition. In our description, we assume a spatial data structure that forms a tree structure, where each tree node represents some region of space and where objects (or pointers to them in external storage) are stored in 
the leaf nodes whose region intersects the objects. Further, we assume that each object is stored in only one leaf. We handle both the case that the objects are stored directly in the leaf as well as the case that the leaf nodes contain the minimum bounding rectangles of objects along with a pointer to the actual object representation. This set of assumptions was chosen as it holds for the R-tree. However, the algorithm can be easily adapted to handle most spatial data structures that do not satisfy these assumptions, such as the hB-tree [23] (which forms a directed acyclic graph), and quadtrees $[26,27]$ (where non-point objects may be stored in more than one leaf node). In the remainder of this section, we do not make a distinction between a node and the region that it represents; the meaning should be clear from the context.

The input to the incremental distance join algorithm is two spatial indexes, $R_{1}$ and $R_{2}$. The algorithm maintains a set of pairs $P$, with one item from each of $R_{1}$ and $R_{2}$, each item being either a node or an object. Initially, $P$ contains just one pair corresponding to the root nodes of $R_{1}$ and $R_{2}$. We obtain the set of all pairs, i.e., the Cartesian product of the sets of objects in $R_{1}$ and $R_{2}$, as follows. As long as $P$ contains a pair $p$ with at least one item being a node, replace $p$ in $P$ by all the pairs resulting from replacing the node by its entries (child nodes for non-leaf nodes, objects for leaf nodes). It should be intuitively obvious that this process will result in $P$ containing the set of all pairs. The algorithm essentially computes $P$ in this way, but processes the pairs in $P$ in order of their distance, thereby attempting to report object pairs as soon as possible.

The algorithm works for data objects of arbitrary type and dimension (although our experiments use two-dimensional points), provided that consistent distance functions are used. Four distance functions are needed: one between objects of each collection, two between objects of one collection and nodes of the spatial index of the other collection, and one between nodes of each spatial index. More accurately, the functions we need are $d_{o o}\left(o_{1}, o_{2}\right)$, $d_{o n}\left(o_{1}, n_{2}\right), d_{n o}\left(n_{1}, o_{2}\right)$, and $d_{n n}\left(n_{1}, n_{2}\right)$, where $o_{1}$ and $n_{1}$ are an object and a node from $R_{1}$, respectively, and $o_{2}$ and $n_{2}$ are an object and a node from $R_{2}$, respectively. If the leaf nodes store minimum bounding rectangle for objects, then the functions $d_{o n}$ and $d_{n o}$ are not required. Instead, we need the functions $d_{b n}\left(b_{1}, n_{2}\right)$, $d_{n b}\left(n_{1}, b_{2}\right)$, in addition to $d_{b b}\left(b_{1}, b_{2}\right)$, where $b_{1}$ and $b_{2}$ denote a minimum bounding rectangle for objects in $R_{1}$ and $R_{2}$, respectively. If node regions are rectangles, then $d_{n n}$ can serve the purpose of all three functions.

Usually, the distance functions are all based on a distance metric for points, $d\left(p_{1}, p_{2}\right)$, such as the Chessboard, Manhattan or Euclidean metrics. However, this need not be the case. As long as the distance functions are "consistent", the algorithm will function correctly. Informally, by consistent, we mean that no pair can have a smaller distance than a pair that gives rise to it during the processing of the algorithm. For example, if $o_{1}$ and $o_{2}$ are objects in $R_{1}$ and $R_{2}$, respectively, and $n_{1}$ is a leaf node that contains $o_{1}$, then we must have $d_{\circ o}\left(o_{1}, o_{2}\right) \geq d_{n o}\left(n_{1}, o_{2}\right)$. If the distance functions are all based on the same metric, this condition will hold due to the triangle inequality property. In what follows, we usually refer to the distance functions collectively with the symbol $d$, as the particular distance function to be used can be inferred from the context.

\subsubsection{Basic Algorithm}

We first describe the basic version of the algorithm, and then introduce extensions to it as well as ways to improve its performance. The heart of the algorithm is a priority queue, where each element contains a pair of items, one from each of the input spatial indexes $R_{1}$ and $R_{2}$. An item can be either a data object or a node, so there are four kinds of possible pairs, node/node, node/object, object/node, and object/object. If object bounding rectangles (abbreviated by $o b r)$ are stored in leaves, then these will become the third type of pair items, resulting in nine possible kinds of pairs, of which we use five: node/node, node/obr, obr/node, obr/obr, and object/object ${ }^{1}$. The key used to order the queue elements is the distance between each pair. We later discuss how to handle ties, i.e., how to order pairs with equal distance.

At each step in the algorithm, the element at the head of the priority queue is retrieved, i.e., the element with the smallest distance key. If the element stores a pair of data objects, then the pair is reported as the next closest pair. No pair that is subsequently reported will have a smaller distance due to this pair having the smallest key in the queue. Furthermore, the consistency constraints on the distance functions guarantee that no pair on the queue will result in generating a pair of data objects with a smaller distance ${ }^{2}$. If one of the items in the dequeued element is a node, then the algorithm pairs up the entries of the node (objects for leaf nodes, child nodes for nonleaf nodes) with the other item.

The basic algorithm is presented in Figure 3 for the case that the leaf nodes of the spatial indexes contain object bounding rectangles. In the figure, item 1 in a queue element is from $R_{1}$, while item 2 is from $R_{2}$. The INCDISTJOIN procedure contains the high level control structure for the algorithm, while procedures PROCESSNODE 1 and PROCESSNODE2 enqueue new pairs for each entry in a node from $R_{1}$ and $R_{2}$, respectively. In lines 6 and 11 of INCDISTJOIN, the next closest pair of objects is reported. The entire state of the algorithm is represented by the priority queue. Thus, at this point, control can be passed to the process that invoked the incremental distance join algorithm, which may or may not decide to retrieve more pairs. If one of the items in the dequeued element is a node, then one of the procedures PROCESSNODE 1 and PROCESSNODE2 is called. This version of the algorithm arbitrarily chooses to call PROCESSNODE 1 if both items are nodes.

In line 4 of PROCESSNODE1, [O] denotes the bounding rectangle of $O$ (note that in practice the object reference must be enqueued along with the bounding rectangle). If the object geometry is represented directly in the leaf nodes, then the actual objects would be used here instead of the bounding rectangles. Also, in this case, the if statement in line 7 of INCDISTJOIN would not be needed.

The connection of the incremental distance join to our incremental nearest neighbor algorithm [18] is easy to see from Figure 3, as PROCESSNODE 1 and PROCESSNODE2 are essentially the same as the basic loop of the nearest neighbor algorithm. In particular, in PROCESSNODE1, item 2 serves the role of the query object.

\subsubsection{Priority Queue Ordering and Tree Traversal}

The key for ordering the priority queue of pairs is the distance between the items. An important question is how to break ties for pairs with the same distance. Different choices will lead to vastly different traversal patterns. Since our goal is to produce result pairs as soon as possible, it is obvious that we want to order pairs containing objects or object bounding rectangles ahead of (i.e., with greater priority than) pairs of nodes. Furthermore, given two pairs with nodes, the pair containing nodes at a deeper level is given a higher priority. This leads to a depth-first-like traversal pattern of the tree hierarchy of the spatial indexes for pairs having the same distance (a version

\footnotetext{
${ }^{1}$ Note that objects only appear in one of the combinations that we allow in order to reduce the number of accesses to the object storage. With our scheme, each object must be accessedat most once for each object/object pair.

${ }^{2} \mathrm{~A}$ pair $\left\langle i_{1}, i_{2}\right\rangle$ is said to be generated from a pair $\left\langle i_{1}^{\prime}, i_{2}^{\prime}\right\rangle$ if the pair $\left\langle i_{1}, i_{2}\right\rangle$ results from a sequence of algorithm operations starting with $\left\langle i_{1}^{\prime}, i_{2}^{\prime}\right\rangle$. As an example, all object/object pairs are ultimately generated from the initial pair of root nodes.
} 


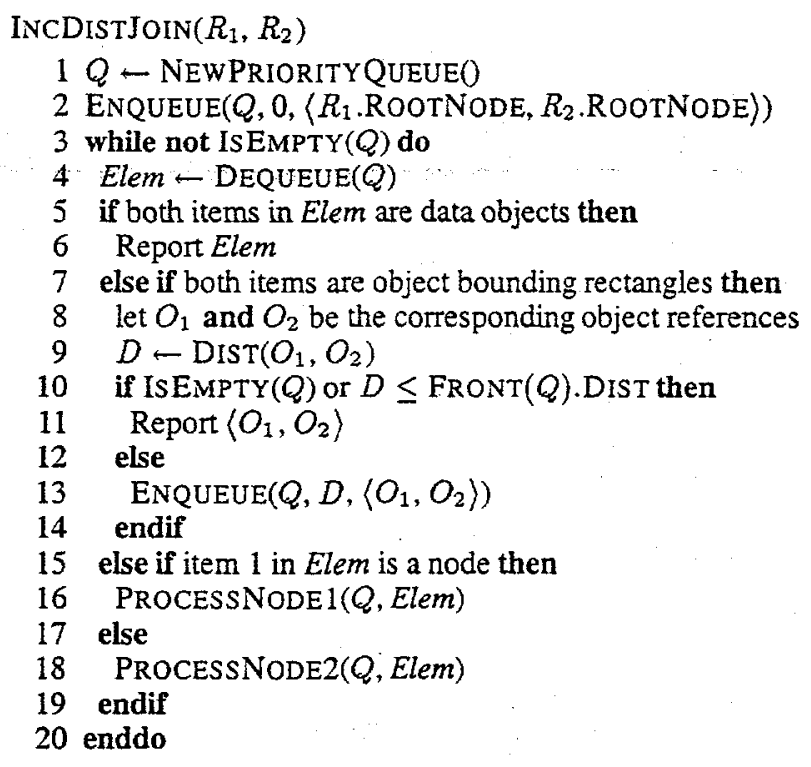

PROCESSNODE $1(Q$, Elem $)$

1 Node $\leftarrow$ item 1 of Elem

2 Item $2 \leftarrow$ item 2 of Elem

3 if Node is a leaf node then

4 for each entry [O] in Node do

5 EnQueue $\left(Q, \operatorname{Dist}\left([\mathrm{O}]\right.\right.$, Item $\left._{2}\right),\left\langle[\mathrm{O}]\right.$, Item $\left.\left._{2}\right\rangle\right)$

6 enddo

7 else

8 for each Child node of Node do

9 ENQUEUE( $Q$, Dist(Child, Item $),($ Child, Item 2$))$

10 enddo

11 endif

PROCESSNODE2(Q, Elem)

1 Same as PROCESSNODE 1 , with items 1 and 2 exchanged

Figure 3: Basic version of incremental distance join algorithm where leaf nodes contain bounding rectangles.

using this approach is termed "DepthFirst" in Section 4.1.1). Alternatively, if nodes at a higher level are given priority, a breadth-firstlike traversal would result (termed "BreadthFirst" in Section 4.1.1). This could be of advantage if we wanted to compute a large portion of the distance join operation (i.e., generate a very large number of pairs), as it would in certain cases enable the algorithm to better schedule node and object accesses [21]. However, given our usage assumptions, much of the work may be wasted, as a breadth-first traversal would require processing all pairs at one level before any pairs with the same distance at the next level are considered.

In the version of the INCDISTJOIN procedure that we presented in Figure 3, when the dequeued pair contains two nodes, $\left\langle n_{1}, n_{2}\right\rangle$, node $n_{1}$ is arbitrarily chosen to be processed (i.e., its entries examined) rather than $n_{2}$. This is not a good strategy, as it will cause $R_{1}$ to be traversed down to the leaf level before the root of $R_{2}$ is processed. A better strategy would attempt to traverse the two indexes more evenly so that the level of the nodes in node/node pairs does not differ by much. This is done by choosing to process the node that is at a shallower depth. If both nodes are at the same level in their respective trees, then the algorithm chooses to process the node whose region has a larger area. Although the strategy that we have outlined is not always the best one, our experiments have shown it to perform well overall.

An alternative to processing only one of the nodes for node/node pairs is to process both simultaneously (termed "Simultaneous" in Section 4.1.1). This is more in line with traditional spatial join algorithms $[8,21]$. In fact, if this is done, then many of the optimization techniques developed for spatial join can be applied [8], such as the usage of plane sweep and the restriction of the search space. The idea is that when processing pair $\left\langle n_{1}, n_{2}\right\rangle$, we first mark the entries in $n_{1}$ that are within the specified distance range (see Section 2.2.3) from the space spanned by $n_{2}$, and similarly for the entries in $n_{2}$ we mark the ones that are within the specified distance range from the space spanned by $n_{1}$. This serves to eliminate entries that cannot possibly become members of any of the new pairs. Next, a plane sweep along one of the axis is used to pair up the entries in the two nodes (which have previously been sorted along that axis). Figure 4 illustrates the plane-sweep process, where $r 1$ and $r 2$ are entries in $n_{1}$, and $s 1, s 2, s 3$ and $s 4$ are entries in $n_{2}$. Without plane sweep, $r 1$ would have to be checked for intersection with all the entries in $n_{2}$, but with plane sweep we only have to check intersection of $r 1$ with $s 1$ and $s 2$. The plane-sweep algorithm given in [8] has to be modified to work for a non-zero maximum distance (recall that [8] focuses on spatial join with the intersection predicate). For example, if the rectangle currently being used has the coordinate range $\left(x_{1}, x_{2}\right)$ along the sweep axis, then the algorithm must sweep along the entries in the other node up to the coordinate value $x_{2}+D_{\max }$, where $D_{\max }$ is the maximum distance. As an example, in Figure 4, we would have to check whether $s 3$ is within the proper distance of $r 1$, in addition to $s 1$ and $s 2$.

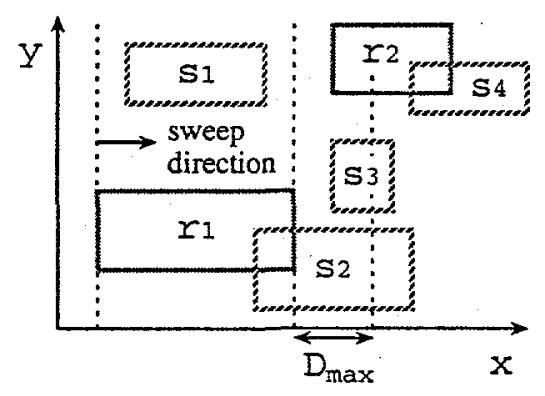

Figure 4: Plane sweep along $x$-axis over the entries in two nodes.

Processing both nodes simultaneously for node/node pairs is not always better than processing only one node as in our original formulation. Intuitively, it seems likely that the optimizations that it affords will only yield significant benefits if the distance range is rather narrow. As an extreme case, if the minimum is 0 and the maximum is unbounded, then all possible pairs of entries from the two nodes will have to be generated, a total of $\left|n_{1}\right| \cdot\left|n_{2}\right|(|n|$ denotes the number of entries in node $n$ ). In contrast, if only one of the nodes is processed, say $n_{1}$, then only $\left|n_{1}\right|$ pairs will result. All of these pairs may have a greater distance than the next closest pair. Thus, in best case, only $\left|n_{1}\right|$ pairs are generated from $\left\langle n_{1}, n_{2}\right\rangle$ with our original formulation of the incremental distance join algorithm before the next object pair is reported. The downside, of course, is that processing only one node at a time may lead to each node being accessed more times from disk when the algorithm has to produce many result pairs.

If both nodes in node/node pairs are processed simultaneously, then the incremental distance join algorithm resembles somewhat the spatial join algorithm introduced in [21]. The difference is that [21] is breadth-first and is limited to finding intersecting object pairs, although it would be straightforward to generalize it to com- 
pute a spatial join with a within predicate (however, unlike with our algorithm, if the pairs are desired in order of distance, then the entire result would have to be computed and sorted before the first pair can be reported).

Some widely used spatial data structures form unbalanced tree hierarchies (e.g., quadtrees [27] and the buddy-tree [28]). Bounding rectangles are not always present in the leaf nodes of these structures, even when objects are not represented directly in the leaves (i.e., the leaves only contain pointers to the objects). If this is the case, then it is better to defer processing leaf nodes until both items in node/node pairs are leaf nodes, at which time both leaf nodes are processed simultaneously. This strategy will tend to reduce the number of times each object needs to be accessed from disk.

\subsubsection{Distance Range}

A shortcoming of the algorithm as stated in Section 2.2.1 is that a very large number of pairs will be inserted into the priority queue, even when computing a modest number of object pairs for relatively small object relations. Most of the pairs inserted in the priority queue will have a large distance, and will most likely never be retrieved from the queue unless a very large number of object pairs is requested. However, for object relations of non-trivial size, the number of pairs in the Cartesian product of the two relations (recall that a full distance join operation computes the Cartesian product) is immense. For example, for two relations with 50,000 objects each, the Cartesian product contains 2.5 billion pairs. Typical queries will only require computing a very small fraction of this high number. Thus, it is unlikely that pairs with a large distance are ever retrieved from the queue. The large number of pairs put on the queue and never requested occupies a great deal of memory space and slows down queue operations ${ }^{3}$. Thus, we need a way of limiting the number of pairs inserted into the queue. One way of doing so is to impose a maximum distance on object pairs. Any pair that has a distance larger than the maximum can be rejected, as no object pair with less distance can be derived from it (this is guaranteed by the consistency of the distance functions).

Above, we have established the need to be able to impose a maximum on the distance of object pairs. In addition, it may be useful for some queries to impose a minimum on the distance of object pairs. The incremental distance join algorithm is easily modified so that it limits the distance of the pairs that are returned to a range of values. In order to effectively prune pairs based on a minimum distance, we need functions that compute an upper bound on the distance of any object pair that can be generated from a pair $\left\langle i_{1}, i_{2}\right\rangle$ (such a function is clearly not needed for object/object pairs). In other words, for any object pair $\left\langle o_{1}, o_{2}\right\rangle$ generated from $\left\langle i_{1}, i_{2}\right\rangle$, we have $d\left(o_{1}, o_{2}\right) \leq d_{\max }\left(i_{1}, i_{2}\right)$, where $d_{\max }$ is the upper bound function appropriate for $\left\langle i_{1}, i_{2}\right\rangle$. This means that if $d_{\max }\left(i_{1}, i_{2}\right)$ is smaller than the minimum distance bound, then we can discard the pair $\left\langle i_{1}, i_{2}\right\rangle$, as no object pair with a distance larger than the minimum will be generated from $\left\langle i_{1}, i_{2}\right\rangle$.

Now, the question is how to compute $d_{\max }$ for the various types of pairs. For pairs of nodes, $\left\langle n_{1}, n_{2}\right\rangle$, we have $d_{\max }\left(n_{1}, n_{2}\right)=$ $\max _{p_{1} \in n_{1}, p_{2} \in n_{2}} d\left(p_{1}, p_{2}\right)$. For a node/object pair $\left\langle n_{1}, o_{2}\right\rangle$, we have $d_{\max }\left(n_{1}, o_{2}\right)=\max _{p_{1} \in n_{1}} d\left(p_{1}, o_{2}\right)$, and similar for object/node pairs. The functions for node/obr, obr/node, and obr/obr pairs can be defined in a similar manner as for node/node pairs. However, a closer approximation to the upper bound is possible for these types of pairs through the use of a distance metric

\footnotetext{
${ }^{3}$ But see Section 3.2 for a description of a priority queue implementation that puts part of the queue on disk if its size is too large to fit in memory.
}

that has been termed MINMAXDIST [25]. The object bounding rectangles are required to minimally bound the objects. The key idea behind the MINMAXDIST metric is that if $b$ is the $d$ dimensional minimum bounding rectangle of object 0 ; then each of the $d-1$ dimensional faces ${ }^{4}$ of $b$ must touch $o$ at some point. Thus, given a point $p$, we have $d(p, o) \leq \max _{p_{f} \in f} d\left(p, p_{f}\right)$, for each $f \in F(b)$, where $F(b)$ denotes the set of faces of $b$. The face $f$ causing the right hand side of the inequality to reach its minimum is the best approximation of $d(p, 0)$ given the bounding rectangle $b$, so the function computing the MINMAXDIST for a point and a bounding rectangle is $d_{\mathrm{mm}}(p, b)=$ $\min _{f \in F(b)}\left(\max _{p_{f} \in f} d\left(p, p_{f}\right)\right)$. A practical way of computing the value of $d_{\operatorname{mm}}(p, b)$ is to first compute the maximum distance from $p$ to a vertex of $b$, say $v_{\max }$, and then to determine the vertex adjacent to $v_{\max }$ (i.e., along an edge) that is closest to $p$ [25]. Now, we can define $d_{\max }\left(n_{1}, b_{2}\right)=\max _{p \in n_{1}} d_{\operatorname{mm}}\left(p, b_{2}\right)$, and similarly for obr/node pairs. The MINMAXDIST definition of $d_{\max }$ for two object bounding rectangles is more complicated: $d_{\max }\left(b_{1}, b_{2}\right)=$ $\min _{f_{1} \in F\left(b_{1}\right), f_{2} \in F\left(b_{2}\right)}\left(\max _{p_{1} \in f_{1}, p_{2} \in f_{2}} d\left(p_{1}, p_{2}\right)\right)$. The price of basing the $d_{\max }$ functions for pairs with at least one bounding rectangle on the MINMAXDIST metric is that they are more expensive to compute than the simpler $d_{\max }$ function for node/node pairs.

Figure 5 presents a version of PROCESSNODE 1 that restricts distances to a range of values. We must also modify the if statement in line 7 of the INCDISTJOIN procedure in Figure 3 to check that the distance $D$ falls in the desired range. The arguments $M i n$ and Max in Figure 5 specify the minimum and maximum desired distance. MINDIST denotes the regular distance functions (i.e., DIST in Figure 3) while MAXDIST denotes the $d_{\max }$ functions. Again, this version of PROCESSNODE 1 assumes that the leaf nodes of the spatial indexes store bounding rectangles. If the object geometry is represented directly in the leaf nodes, then the actual objects would be used in line 4 of Figure 5. Also, in that case, if item 2 is an object, then MAXDIST is equivalent to MiNDistin line 5.

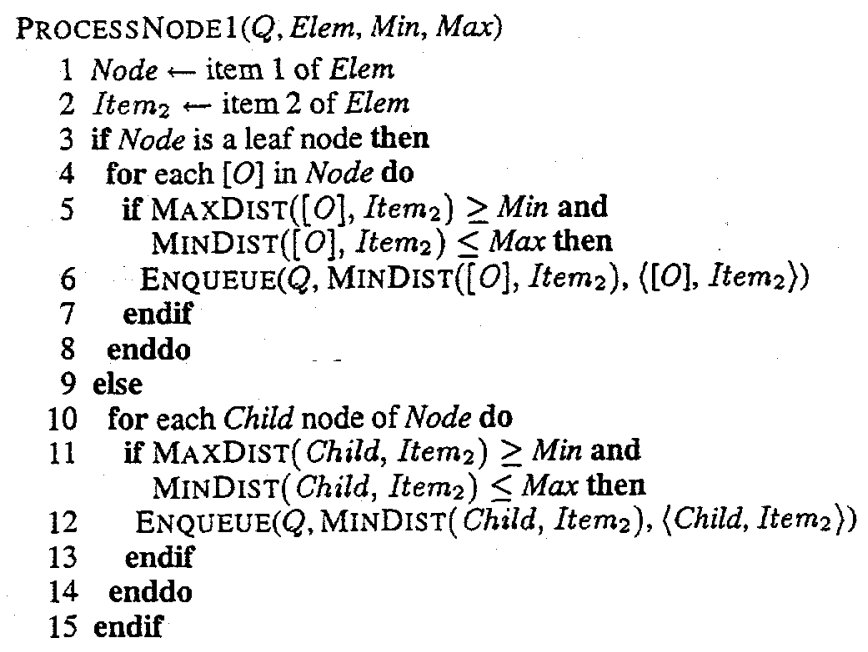

Figure 5: Portion of incremental distance join algorithm with distance range restriction.

\footnotetext{
${ }^{4}$ In two dimensions, the faces are line segments.
} 


\subsubsection{Estimating Maximum Distance}

As we pointed out in Section 2.2.3, a reasonably narrow distance range (i.e., small interval between minimum and maximum distance) is crucial for the incremental distance algorithm to perform well. However, it is often not practical to require the user to set a maximum distance. Furthermore, the maximum distance is likely to be greatly overestimated. It is therefore important to have another way of estimating the maximum distance, given some other information. One way of doing so is to set an upper bound on the number of pairs that the algorithm must compute. In many applications, especially involving interactive queries, a fairly low number of pairs are known to be needed. This is aided by query language extensions that enable limiting the number of tuples in the result of queries (e.g., the "STOP AFTER" clause proposed for the "SELECT" statement of SQL [10]).

Given that the algorithm must compute a maximum of $K$ pairs, the algorithm can estimate the maximum distance based on the pairs that have been seen so far. Obviously, if $K$ object/object pairs have been seen, then the pair with the largest distance among those $K$ pairs will provide a lower bound on the maximum distance necessary to compute the $K$ closest pairs. However, we can do better than this by also making use of other types of pairs (e.g., node/node pairs). In general, more than one object/object pair may be generated from a pair $\left\langle i_{1}, i_{2}\right\rangle$. This means that much fewer than $K$ pairs are sufficient for estimating the maximum distance of $K$ object/object pairs.

In the following, $D_{\min }$ and $D_{\max }$ denote the minimum and maximum distance imposed on the pairs to be computed by the algorithm, $d$ denotes a regular distance function (i.e., computing minimum distance between two items) and $d_{\max }$ denotes the functions computing the upper bound on the distance of any object pairs generated from a pair. If the query specifies no maximum on the distance, then $D_{\max }$ is initially $\infty$. Our goal is to reduce $D_{\max }$ as much as possible, given $K$, the maximum number of pairs requested. Whenever a pair $\left\langle i_{1}, i_{2}\right\rangle$ is inserted into the priority queue, we show below how to use the pair for the purpose of estimating a lower value for $D_{\max }$. Doing this adds overhead to the algorithm, but unless $K$ is very large, it reduces considerably the number of pairs inserted into the priority queue, and thereby improves the overall running time of the algorithm.

A pair $\left\langle i_{1}, i_{2}\right\rangle$ is eligible to be used for estimating $D_{\max }$ if $d\left(i_{1}, i_{2}\right) \geq D_{\min }$ and $d_{\max }\left(i_{1}, i_{2}\right) \leq D_{\max }$. This guarantees that all object/object pairs generated from $\left\langle i_{1}, i_{2}\right\rangle$ will have a distance in the range $\left[D_{\min }, D_{\max }\right]$. Since we cannot know in advance how many object pairs are generated from a pair $\left\langle i_{1}, i_{2}\right\rangle$, we must instead determine a lower bound on this number. This can be derived from the minimum number of objects in the subtree of $i_{1}$ and $i_{2}$, assuming they are nodes (if they are objects or object rectangles, this number is one). The minimum number of objects in the subtree of a node can, in turn, be derived from the minimum fan-out and the height of the corresponding tree. For the R-tree, for example, the minimum fan-out of nodes is typically $40 \%$ of the maximum fan-out (except for the root node). A more aggressive strategy would result from using the expected number of pairs generated from $\left\langle i_{1}, i_{2}\right\rangle$ based on the average node occupancy. However, if the number of pairs generated from $\left\langle i_{1}, i_{2}\right\rangle$ is over-estimated, then this may lead to a value of $D_{\max }$ that is too small (i.e., smaller than the $K^{\text {th }}$ object/object pair), thereby causing us to find less than $K$ pairs which will force us to restart the query. The reason we need to restart is that the priority queue does not provide us any useful information as we will have pruned too many entries by our maximum distance heuristic.

The process for estimating $K$ maintains a set of pairs $M$, each of which has been inserted in the priority queue but not retrieved from it. When an eligible pair (i.e., with the distance function values as specified above) is inserted into the priority queue, it is also inserted into $M$. If this causes the sum of the number of object/object pairs (actually, the lower bound of object/object pairs as described above) that can be generated for the pairs in $M$ to be larger than $K$, then we remove pairs from $M$ until this is not the case, setting $D_{\max }$ to the $d_{\max }$ value of the pair removed last. The pair to remove next is chosen based on the largest $d_{\max }$ value. When a pair is retrieved from the priority queue, we must also remove the pair from $M$ if it is present. However, when reporting the next object/object pair, we can reduce the value of $K$ by one.

The question is how to organize the set $M$. The operations that are performed on $M$, in addition to insertion, are to remove the pair with largest $d_{\max }$ as well as to remove a pair given the particular items in the pair. There is no single data structure that supports efficient execution of both of these operations. In our implementation, we chose to use a priority queue $Q_{M}$ organized on the $d_{\max }$ values to support finding the largest value, and a hash table to support locating a particular pair. The hash table entries contain a pointer to the corresponding priority queue entry, thereby enabling deleting the entry from $Q_{M}$ for a pair that must be removed. It is important not to confuse $Q_{M}$ with the main priority queue of the algorithm (i.e., $Q$ in Figure 3). $Q_{M}$ will not be discussed further in the remainder of this paper.

\subsubsection{Other Extensions}

A number of other extensions of the incremental distance join are possible. The first is to add some spatial criterion to one or both of the relations involved in the join. As an example, the objects may be required to fall inside a given rectangle, or they may be required to have some minimum area. Such an extension can actually be applied equally to other spatial join algorithms, and does not necessarily involve modifying the algorithm. Instead, the distance functions (which may be parametrized) can check the additional spatial criteria, and return some special value if the pair should be discarded. Of course, if the spatial criterion has a high selectivity (i.e., such that few objects in each relation participating in the join satisfy the criterion), then it may be better to first restrict the number of objects by using the spatial criterion before computing the join. However, the cost of that alternative will include building a spatial index on the resulting restricted relations, or it will require using some algorithm other than the incremental distance join. In either case, it may take longer to produce the first few pairs with the alternative than with the incremental distance join, since it it highly geared towards producing pairs early.

The second extension is to impose a secondary ordering on pairs produced by the algorithm, besides the distance between the objects. This is probably most useful if the resulting pairs are required to intersect, i.e., the maximum distance is 0 . For example, we may wish to find the intersections of roads and rivers in order of distance from a given house. In the general case, this extension requires modifying the algorithm. However, for the special case of finding intersections, the distance functions could return $\infty$ for nonintersecting pairs, but for intersecting pairs, the functions would return some ordering value (such as the distance from the house in our example).

Another possible extension is to find the pairs in reverse order of distance, i.e., the farthest pair first, etc. This is relatively simple to achieve. Instead of ordering the elements on the priority queue in ascending order of distance, we would order them in descending order of distance (for example, this can be done by simply using the negative of the distance as a key). In addition, instead of using the regular distance functions as a key to order the pairs on the priority queue, the $d_{\max }$ functions must be used for all types of pairs except object/object pairs (recall that the $d_{\max }$ functions compute an upper bound on the distance of object/object pairs generated from pairs). 
As before, the algorithm will perform better if the distance range is rather narrow. However, in this case, we can estimate the minimum distance in the presence of an upper bound $K$ on the number of object pairs that will be requested. This is instead of estimating the maximum distance as was described in Section 2.2.4.

\subsection{Computing Distance Semi-Join}

Recall that distance semi-join is a subset of a distance join, where an object pair $\left\langle o_{1}, o_{2}\right\rangle$ appears in the result only if none of the prior pairs contain $o_{1}$ as the first item. Thus, we must keep track of the set $S_{o}$ of objects $o_{1}$ whose pairs $\left\{o_{1}, o_{2}\right\rangle$ have been reported. The easiest way to extend the incremental distance join algorithm to compute a distance semi-join is to use the algorithm unchanged and check outside of the algorithm if object $o_{1}$ in output pairs $\left\langle o_{1}, o_{2}\right\rangle$ has been seen before (i.e., if it is present in the set $S_{\circ}$ ). However, that approach (termed "Outside" in Section 4.2.1) does not take advantage of the special structure of the distance semi-join to reduce the amount of work expended by the algorithm. In this section we identify several possible ways to modify the incremental distance join algorithm such that it computes the distance semi-join operation more efficiently. Also, we discuss how the extensions and optimizations described in section 2.2 for the incremental distance join algorithm apply for computing the distance semi-join operation.

First, we must bring into the algorithm the knowledge of the set $S_{o}$, the set of objects from the first collection that has already been seen. This is straightforward to do, and requires minor modifications to the INCDISTJOIN procedure of Figure 3 (termed "Inside1" in Section 4.2.1) as well as to procedure PROCESSNODE1 (termed "Inside2" in Section 4.2.1). Specifically, in line 4 of INCDISTJoIN, if in the dequeued element $\left\langle i_{1}, i_{2}\right\rangle, i_{1}$ is an object or an object bounding rectangle, then we check if $i_{1}$ is present in $S_{o}$. If so, then we discard the pair. In PROCESSNODE 1, if the node is a leaf node, then in line 5 we must ignore entries that correspond to objects that are present in $S_{\circ}$.

Bringing the knowledge of the set $S_{\circ}$ into the algorithm is a definite improvement, but we can do better still. The next improvement is based on the fact that for each pair $\left\langle O_{1}, O_{2}\right\rangle$ in the output of the distance semi-join of $A$ and $B, o_{2}$ is the object in $B$ nearest to $o_{1}$. This can be exploited locally in the PROCESSNODE2 procedure (termed "Local" in Section 4.2.1). To see how, note that for a pair $\left\langle o_{1}, n_{2}\right\rangle$, the object in the subtree of $n_{2}$ closest to $o_{1}$ is most likely in some of the entries of $n_{2}$ whose region is near $o_{1}$. Specifically, we compute $d_{\max }\left(o_{1}, e_{2}\right)$ for each entry $e_{2}$ in $n_{2}$, and determine the minimum value, $D_{\mathrm{mm}}$. Any entry in $n_{2}$ that is farther away from $o_{1}$ than $D_{\mathrm{mm}}$ can be discarded as it is guaranteed not to contain the object in the subtree of $n_{2}$ nearest to $0_{1}$. This principle can be applied in PROCESSNODE2 even for pairs $\left\langle i_{1}, n_{2}\right\rangle$ where $i_{1}$ is an object bounding rectangle or a node, since $d_{\max }\left(i_{1}, n_{2}\right)$ is an upper bound on the distance of any object pair derived from $\left\langle i_{1}, n_{2}\right\rangle$. Observe that this approach is analogous to the downward pruning strategy of the nearest neighbor algorithm of [25].

A more aggressive strategy can be obtained by using the same insight in a global fashion. In other words, for each object and node in $R_{1}$ (the spatial data structure representing the objects in $A$ ), maintain the smallest $d_{\max }$ distance that has been seen so far (termed "GlobalAll" in Section 4.2.1). Any time we consider enqueuing a pair $\left\langle i_{1}, i_{2}\right\rangle$, we would first make sure that the distance of the pair is smaller than the smallest $d_{\max }$ distance for $i_{1}$. Employing this strategy requires a considerable amount of memory space if $R_{1}$ contains many objects. Nevertheless, it is useful as a comparison with the other strategies. Moreover, we can compromise by only maintaining the globally smallest $d_{\max }$ distance for the nodes of $R_{1}$, which requires an order of magnitude less space than doing so also for the objects in $R_{1}$ (termed "GlobalNode" in Section 4.2.1).
As in the case of computing the distance join incrementally, we can estimate the maximum distance needed to produce a maximum of $K$ pairs for the distance semi-join. This is done in much the same way as described in Section 2.2.4. The difference, here, is that in the set $M$ of the pairs being used in the estimation process, the first item in each pair is unique. In other words, if $\left\langle i_{1}, i_{2}\right\rangle$ is a pair in $M$, then no other pair in $M$ has $i_{1}$ as the first item ${ }^{5}$. Also, the number of pairs generated from a pair $\left\langle i_{1}, i_{2}\right\rangle$ is bounded by the number of objects in the subtree of $i_{1}$, assuming $i_{1}$ is a node. When an item $\left\langle i_{1}, i_{2}\right\rangle$ is about to be inserted into $M$, we must first check if another item $\left\langle\dot{i}_{1}, i_{2}^{\prime}\right\rangle$ exists in $M$. If so, then we replace $\left\langle i_{1}, i_{2}^{\prime}\right\rangle$ by $\left\langle i_{1}, i_{2}\right\rangle$ if the latter has a smaller $d_{\max }$ value and ignore $\left\langle i_{1}, i_{2}\right\rangle$, otherwise. There are two additional subtle differences. When $\left\langle o_{1}, o_{2}\right\rangle$ is reported, any pair $\left\langle o_{1}, i_{2}\right\rangle$ in $M$ must be removed. Also, a pair $\left\langle n_{1}, i_{2}\right\rangle$ may only be inserted into $M$ if $n_{1}$ has never been processed for any pair $\left\langle n_{1}, i_{2}^{\prime}\right\rangle$. Otherwise, some of the objects in the subtree of $n_{1}$ would be counted more than once (since processing $n_{1}$ in the pair $\left\langle n_{1}, i_{2}^{\prime}\right\rangle$ may lead to some pairs $\left\langle e_{1}, i_{2}^{\prime}\right\rangle$ to be inserted into $M$ where $e_{1}$ is an entry in $\left.n_{1}\right)$. This may lead to an estimate of $D_{\max }$ that is too low thereby causing us to find less than $K$ pairs which forces us to restart the query. The reason we need to restart is that the priority queue does not provide us any useful information as we will have pruned too many entries by our maximum distance heuristic.

The extensions discussed in Section 2.2 .5 also apply for the distance semi-join version of the our incremental algorithm. However, modifying the algorithm to find pairs in reverse order of distance leads to what may seem an unintuitive, and perhaps not very useful, result. There are two possible ways of defining a reverse distance semi-join operation on relations $A$ and $B$. The first is to report in reverse order of distance the object in $B$ closest to each object in $A$. The second is to report in reverse order of distance the object in $B$ farthest from each object in $A$. The straightforward way of applying the incremental distance join to the reverse distance semi-join will be in accordance to the second definition since it corresponds to reporting for each object $o_{1}$ the first pair $\left\langle o_{1}, o_{2}\right\rangle$ that occurs in a reverse distance join. The first definition would mean reporting for each object $o_{1}$ the last pair $\left\{o_{1}, o_{2}\right\}$ that occurs in a reverse distance join, which would be extremely inefficient.

\section{Experimental Environment}

\subsection{System and Data}

All of our experiments were run on a Sun Ultra 1 Model 170E machine, rated at 6.17 SPECint95 and 11.80 SPECfp95, with 64MB in main memory and a $2.1 \mathrm{~GB}$ internal disk drive. The spatial data structure that we used is an $R^{*}$-tree [5]. The size of the nodes was $1 \mathrm{~K}$, for a maximum fan-out of 50 , with $256 \mathrm{~K}$ of memory used for buffers. The spatial objects were represented directly in the leaves of the $\mathrm{R}^{*}$-trees. We chose that approach in order to simplify the analysis of the execution time results. Also, the organization of the external object storage has a large effect on the performance, and thus introduces an additional variable. The software was compiled with a GNU $\mathrm{C}++$ compiler set for maximum optimization $(-03)$. The distance functions were based on the Euclidean metric.

As in other evaluations of spatial algorithms (e.g., [8, 21]), we derived our test data from the TIGER/Line File [9]. We used two sets of points from the coverage of the Washington, DC area: Water contains the centroids of water features $(37,495$ points), and Roads contains the centroids of road features ( 200,482 points). It should be

\footnotetext{
${ }^{5}$ As far as $M$ is concerned, an object bounding rectangle is treated in the same way as the corresponding object; both are represented by the object identifier.
} 
clear that dealing with line data is much more complex than points. Making experiments with line data and more complex spatial features is a subject for future study.

\subsection{Implementation Details}

An important issue is the implementation of the priority queue. It should be clear that the number of object pairs in the result of a full distance join operation is extremely large, or the same as in the Cartesian product of the relations (in the absence of distance range restrictions). Even when computing the entire distance join (this is not likely to be very useful in practice, however), the size of the priority queue in the incremental distance join algorithm remains much smaller than the size of the result. Nevertheless, a small fraction of a very large number is still a large number. Thus, the size of the priority queue may be too large to fit in memory. However, an exclusively disk-based scheme for representing the priority queue is not desirable, due to poor performance.

In our experiments, we use a simple hybrid memory/disk scheme that stores parts of the priority queue in a memory-based heap structure (we chose the pairing heap structure [13]), while the rest is offloaded to disk. If a relatively small number of object pairs is requested, then the vast majority of pairs put on the priority queue will never be needed. Thus, our goal in developing the scheme was that the contents of the priority queue that were put on disk would only be needed when a large number of object pairs were requested. Another reason for limiting the contents of the memory-based heap to pairs that are likely to be needed is that the algorithmic complexity of heap operations is directly related to the size of the heap. We chose to use a three-tiered scheme for representing the priority queue, based on the distance of the pairs. Pairs with a distance less than $D 1$ are stored in the memory-based heap, pairs with a distance less than D2 are stored in an unorganized list in memory, while pairs with a distance of $D 2$ or greater are stored on disk. If the heap becomes empty, then the contents of the unorganized list is put into the heap, the value of $D 1$ is changed to $D 2$, a new value is chosen for D2, and pairs on disk with distance between the new values of $D 1$ and $D 2$ are put into the unorganized list (actually, we avoid accessing the pairs on disk unless they need to be inserted into the priority queue). In our implementation, a fixed distance increment $D_{T}$ is used to update D1 and D2, with their initial values being $D_{T}$ and $2 D_{T}$, respectively. The part of the queue stored on disk is organized in linked lists of pages with the pairs in each list having distances in the range $\left[k D_{T},(k+1) D_{T}\right)$.

The drawback of our priority queue scheme is that it depends on a fixed constant $D_{T}$ rather than responding dynamically to the distribution of the queue contents. In the experiments, we chose a value for $D_{T}$ that worked well for the input relations. Developing a way of choosing $D_{T}$ based on the input relations, or finding some other dynamic method of deciding what part of the priority queue is stored on disk, are subjects for further investigation.

In Section 2.3, a set $S_{o}$ is maintained of objects from $A$ for whom a pair has been reported by the incremental algorithm for the distance semi-join. In our experiments, we use a bit string representation for $S_{o}$. The reason is that a bit string representation is extremely efficient, both for membership tests and insertions. There is certainly a space/time tradeoff involved, since a bit string representation of a set occupies a fixed amount of space, regardless of the size of the set. For sets of only a few elements, it would be much more space efficient to use some other approach. Nevertheless, given the memory capacity of modern computers, the size of the bit strings is modest even for large data sets. For example, a bit string representation of a subset of 1 million elements would occupy 122K.

\section{Performance Results}

In this section, we evaluate the effectiveness of the strategies presented for enhancing the efficiency of the incremental distance join algorithm, as well as compare its performance to competing approaches for computing the distance join and distance semi-join. In the experiments, we joined Water with Roads, except where noted ${ }^{6}$.

\subsection{Distance Join}

\subsubsection{Priority Queue Ordering and Tree Traversal}

Section 2.2.2 discussed the effect of choosing a different priority queue ordering (i.e., how ties are resolved for pairs with the same distance) as well as how to process pairs of two nodes. Table 1 lists the values of some performance measures (the number of object distance calculations, the maximum queue size, and the number of node $\mathrm{V} / \mathrm{O}$ operations) for producing up to 100,000 result pairs of the distance join. The algorithm version used in these experiments was such that pairs with the same distance are ordered so that the algorithm performs a depth-first traversal (i.e., nodes at a deeper level are given priority); only one node is processed at a time in node/node pairs; and the two spatial indexes are traversed evenly ${ }^{7}$. In all experiments below, except where otherwise noted, this type of queue order and traversal is used. In Figure 6, we plot the execution times of this version (labeled "Even/DepthFirst") against three other versions: (1) "Even/BreadthFirst" orders pairs with the same distance such that it leads to breadth-first traversal; (2) "Basic/DepthFirst" is the basic algorithm of Figure 3, where we always process the first node in node/node pairs; and (3) "Simultaneous/DepthFirst" where both nodes of node/node pairs are processed simultaneously.

Overall, the shape of the graphs is similar. For the versions using the priority queue order leading to depth-first traversal ("DepthFirst", "Basic" and "Simultaneous"), obtaining the first pair is relatively inexpensive, while the cost does not rise much for between 10 and 10,000 pairs. However, for computing a larger number of pairs, the cost rises dramatically.

The difference in execution times for the four versions is due to differences in the values of all performance measures in Table 1. However, the dominant factor, although not shown here, is the number of distance calculations and the size of the priority queue, which are much larger for "Basic" and "Simultaneous". Since a maximum distance is not specified for these experiments, the "Simultaneous" version is not able to benefit from its filtering and plane-sweep techniques. The reason for "DepthFirst" being somewhat faster than "BreadthFirst" for retrieving one pair is that there is one object pair with a distance of 0 . This pair is reported as soon as it is found by "DepthFirst", but in "BreadthFirst" it is only reported after all intersecting nodes have been processed. After the first pair, the difference between these methods is negligible.

An interesting question is what the reason is for the sharply higher cost for computing 100,000 pairs compared to computing 10,000 pairs. Table 1 reveals that there is a relatively larger increase in node $1 / O$ between computing 10,000 and 100,000 pairs

\footnotetext{
${ }^{6}$ Since the distance join is symmetric, the result of joining Roads with Water is the same. However, the incremental distance join algorithm is not necessarily symmetric in its execution pattern, so that the execution time may be different based on the order of the joined relations. The distance semi-join operation is not symmetric, so that result of a distance semi-join of Roads with Water is different from a distance semi-join of Water with Roads.

${ }^{7}$ Recall that by traversing evenly we mean that if the nodes in a node/node pair are at a different level in their respective trees, then we choose to process the node at a shallower level.
} 


\begin{tabular}{|r|r|c|c|c|}
\hline \multicolumn{1}{|c|}{ Pairs } & $\begin{array}{c}\text { Time } \\
(\text { sec.) }\end{array}$ & $\begin{array}{c}\text { Dist. } \\
\text { Calc. }\end{array}$ & $\begin{array}{c}\text { Queue } \\
\text { Size }\end{array}$ & $\begin{array}{c}\text { Node } \\
\text { yO }\end{array}$ \\
\hline 1 & 6.9 & 307994 & 1002536 & 3019 \\
10 & 9.0 & 393758 & 1333856 & 4087 \\
100 & 9.4 & 395780 & 1356985 & 4652 \\
1,000 & 9.8 & 403281 & 1434160 & 6487 \\
10,000 & 12.6 & 422392 & 1632895 & 11502 \\
100,000 & 23.8 & 479262 & 2229874 & 28356 \\
\hline
\end{tabular}

Table 1: Values of performance measures for incremental distance join algorithm using depth-first traversal, processing one node at a time, and using even traversal.

(node $/ / O$ counts the number of times a requested node is not in the node buffer). The number of node accesses (not shown in the table) increases by about $43 \%$, and almost all of the additional accesses are for nodes that are not present in the node buffer. A larger node buffer, or a better buffer strategy, will most likely improve the performance for computing 100,000 pairs. Another factor in the higher cost of computing 100,000 pairs is that for that many pairs, parts of the priority queue contents that were written to disk must be read back into memory.

The values of the performance measures when joining Roads with Water, instead of Water with Roads, is virtually the same for these versions of the algorithm, except for "Basic". Since Roads is larger, many more pairs are generated (in this case, Roads is traversed first). In fact, for producing result 100,000 pairs, too many pairs were generated for the priority queue to fit on disk. Thus, the treatment of node/node pairs in "Basic" is clearly too simplistic.

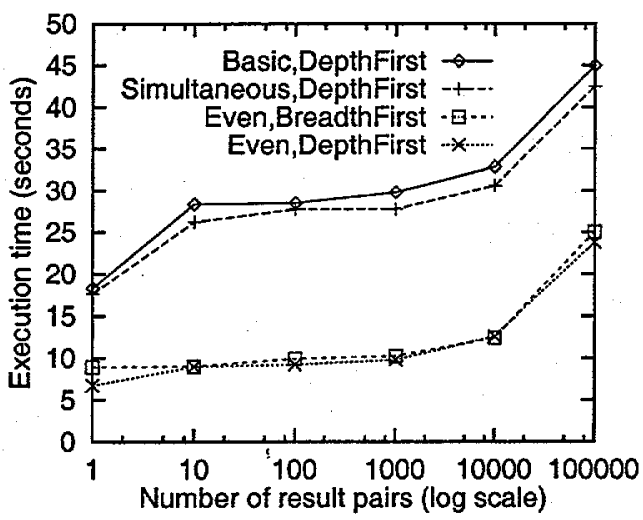

Figure 6: Execution time for different queue order and node processing.

\subsubsection{Maximum Distance and Maximum Pairs}

In Section 2.2.3 we discussed the importance of imposing a maximum distance, and in Section 2.2.4 we described how the maximum distance can be estimated based on an upper bound on the number of object pairs that will be requested. Figure 7 compares the execution time of the regular algorithm (i.e., "DepthFirst" from the preceding section) to two versions of the algorithm applied to distance join: (1) "MaxDist" is the regular algorithm with maximum distance set to the distance of pair number 1000,10,000, and 100,000 (for "MaxDist 1000", we only compute up to 1000 pairs, etc.); (2) "MaxPair" uses the maximum distance estimation for an upper bound of
1000 and 10,000 pairs (setting the maximum to 100,000 was slower than the "Regular" version). The purpose of showing the "MaxDist" plots is to demonstrate the effect of setting the maximum distance, and it also provides a useful benchmark of the effectiveness of the maximum distance estimation of "MaxPair". Of course, in practice we will not know in advance the distance of pair number 1000 , etc.

Figure 7 confirms the benefit of setting the maximum distance. The performance was very similar for the three values for the maximum distance. Setting the maximum number of pairs is seen to be only beneficial for a relatively small number of pairs. For a maximum of 1000 pairs, we get a similar performance as for setting the maximum distance. When the maximum is set to 10,000 pairs, there is less benefit, as the maximum distance estimate is not as tight and the overhead of the estimation process is greater.

In Section 4.1.1 we confirmed that processing both nodes simultaneously for node/node pairs is worse than processing only one at a time if no maximum distance is specified. We performed the same experiments as shown in Figure 7 using the "Simultaneous" version of the incremental distance join algorithm. Although we do not explicitly present these results here, as expected, the performance of "Simultaneous" was better than that of "DepthFirst" when a relatively small maximum distance was specified, or up to $20 \%$ for "MaxDist 1000". However, the improvement was most pronounced for retrieving only a few pairs, and was much smaller for retrieving 10 or more pairs, or usually about $3-5 \%$. Specifying a maximum on the number of pairs was also a little faster using the "Simultaneous" version for a very small number of pairs. For 10 pairs or more, however, it proved better to process only one node at a time in node/node pairs, although the improvement was not great (typically $\approx 2-4 \%$ ).

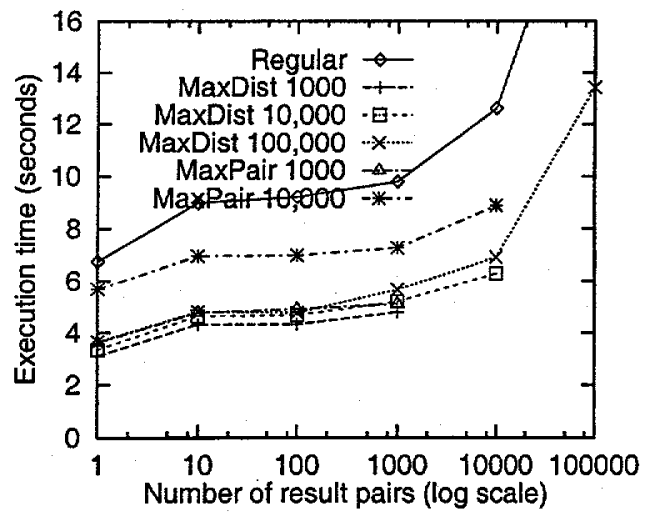

Figure 7: Execution time for different maximum distance and maximum pairs for distance join.

\subsubsection{Priority Queue Implementation}

In Section 3.2 we discussed a hybrid implementation of the priority queue that offloads parts of the queue to disk. Figure 8 gives the execution time for a purely memory-based queue implementation as well as the hybrid one, where two different values of $D_{T}$ are used for the hybrid approach ${ }^{8}$. The memory-based queue is only a little slower for up to 10,000 pairs. However, for 100,000 pairs, it is almost an order of magnitude slower, due to excessive virtual memory thrashing, taking over 180 seconds to compute. The hybrid approach performed almost equally well for the different values of $D_{T}$, except when retrieving 100,000 pairs. In that case, the

\footnotetext{
${ }^{8}$ The values of $D_{T}$, chosen somewhat arbitrarily, correspond to the distances of pairs number 7,663 and 34,906. The latter value was used for all the other experiments.
} 
higher $D_{T}$ value (i.e., "Hybrid2") was better, most likely because it required fewer reads from the disk portion of the priority queue. For fewer than 100,000 pairs, the lower $D_{T}$ value (i.e., "Hybrid1") gave slightly better performance, as a higher number of unnecessary priority queue elements were kept out of the memory based part of the queue. The best value for $D_{T}$ depends both on the nature of the data sets and the amount of available memory.

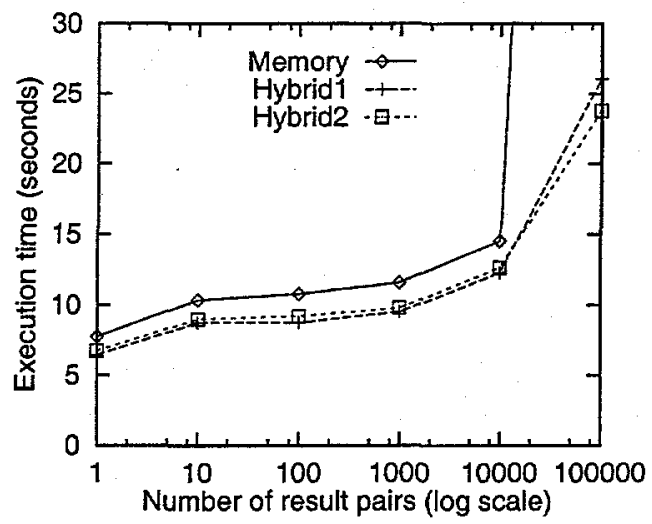

Figure 8: Execution time for storing the priority queue entirely in memory vs. offloading parts on disk.

\subsubsection{Alternative Implementations}

The distance join operation can be computed in other ways than with the incremental distance join algorithm. If a maximum distance is imposed, then a spatial join with a within predicate can be executed, with the output being sorted once it is done. If no maximum distance is imposed, then some distance must be guessed at if an algorithm for the spatial join with within predicate is to be used. If the distance is too small and not enough pairs result, then the spatial join must be executed again with a larger distance. Due to this problem, we do not use a spatial join algorithm for comparison.

Another way of computing a distance join is to use a nested loop approach and compute the distance between all possible pairs of objects. However, this will not compare favorably with using the incremental distance join algorithm unless a very large number of pairs is needed, which is unlikely to arise in practice (for example, the full join for our data sets contains about 7.5 billion pairs). Nevertheless, we did an experiment:with this approach using the Water and Roads data sets. For simplicity sake, we only computed the distance values but didn't store them nor did we sort at the end, which would be necessary for a real implementation. The data set of the inner loop was read completely into memory in order to avoid rereading it. The time to execute the experiment was over $31 / 2$ hours. In that amount of time, the incremental distance join is able to compute at least 100 million pairs. Unfortunately, for that many pairs, the priority queue becomes so large that the incremental distance join is not practical unless a very large disk space is available. However, a large disk space would also be required to generate and sort 100 million pairs using the nested loop approach.

\subsection{Distance Semi-Join}

In this section we discuss some results of our experiments for computing the distance semi-join with variants of the incremental distance join algorithm. Since we are joining Water with Roads, this results in finding the nearest neighbors of points in Water.

\subsubsection{Pair Filtering and Smallest $d_{\max }$ Distance}

In Section 2.3 we enumerated several ways of filtering out pairs $\left\langle i_{1}, i_{2}\right\rangle$ where $i_{1}$ is an object or an object bounding rectangle and $i_{1}$ has already been reported. Also, we presented ways of limiting the number of pairs generated based on the $d_{\max }$ distance of pairs. Figure 9 gives the execution time for these various filtering methods: (1) "Outside" executes the regular incremental distance join algorithm and filters out resulting pairs that contain objects that have already been reported; (2) "Insidel" filters only in the INCDISTJOIN procedure of Figure 3; and (3) "Inside2" filters also in the PROCESSNODE 1 procedure. There are three schemes that exploit the $d_{\max }$ distance, all of which use the filtering of "Inside2": (1) "Local" only works locally in the PROCESSNODE 1 procedure; (2) "GlobalNodes" uses the local strategy, as well as globally maintaining the smallest $d_{\max }$ distance of nodes; and (3) "GlobalAll" globally maintains the smallest $d_{\max }$ distance of both nodes and objects.

Filtering pairs outside the INCDISTJOIN procedure appears to be slightly better for up to 1000 pairs. However, the priority queue became too large to find the neighbors of all points in Water and thus is not shown beyond 10,000 pairs. Filtering inside INCDISTJoIN and/or PROCESSNODE 1 saves some distance calculations and node accesses for retrieving 1000 or more pairs, but this was outweighed by more member checks against the $S_{o}$ set, at least for up to 1000 pairs. For more pairs the benefit of more filtering becomes greater, and for finding the neighbors of all points in Water "Inside1" is about $47 \%$ slower than "Inside2" (530 vs. 362 seconds; this is not shown in Figure 9 in order not to obscure the time difference for smaller numbers of pairs).

The three schemes for exploiting $d_{\max }$ distances also are very similar for up to 10,000 pairs. However, for much larger number of pairs, the benefit of maintaining the $d_{\max }$ distance of all objects and nodes ("GlobalAll") becomes more pronounced. Doing it only for nodes ("GlobalNode") did not seem to result in appreciable improvement compared to "Local".

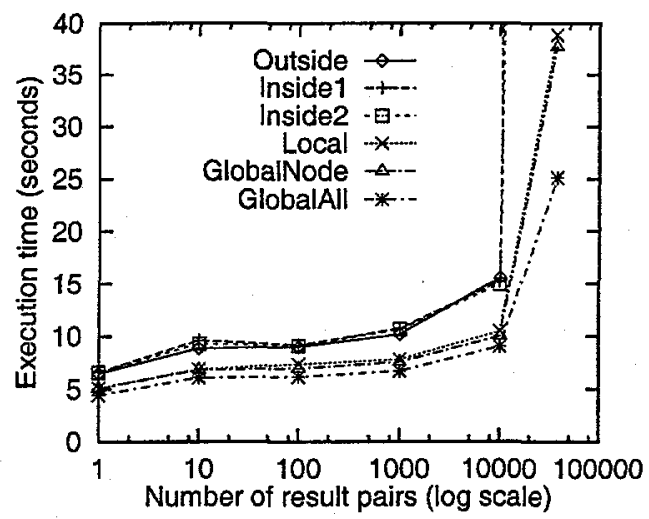

Figure 9: Execution time for storing priority queue entirely in memory vs. offloading parts on disk.

\subsubsection{Maximum Distance and Maximum Pairs}

As in Section 4.1.2, we now report on experiments testing the effect of setting a maximum distance or an upper bound on the numbers of pairs for computing the distance semi-join operation with the incremental distance join algorithm. Figure 10 shows the result of doing this using the "Local" version of Section 4.2.1. In the figure, "MaxDist All" is the result of setting the maximum distance to be the largest possible distance between two objects in the result of the dis- 
tance semi-join and for "MaxPair All", the upper bound on the number of pairs is set to the number of points in Water. The figure confirms the benefit of restricting the maximum distance. Notice that setting the maximum number of pairs to 1000 does indeed improve the execution time, making it virtually identical to setting the maximum distance to the distance of the $1000^{t h}$ pair. However, choosing 10,000 or more as the maximum number of pairs makes the algorithm slower, as such a large limit does not give a tight estimate for the maximum distance, and the overhead cost incurred in estimating the maximum distance exceeds its benefit. The cost of computing the neighbor of all points in River (not shown in the figure) is about 35 seconds for "MaxDist All" and 44 seconds for "MaxPair All". These numbers are about $14 \%$ lower and $13 \%$ higher, respectively, than when maximum distance is not set. Thus, we can see that imposing a maximum distance or setting an upper bound on the number of pairs to be generated only yields significant savings in execution time when the maximum is low (up to $50 \%$ savings or more), while high values on the maximum yields little if any savings.

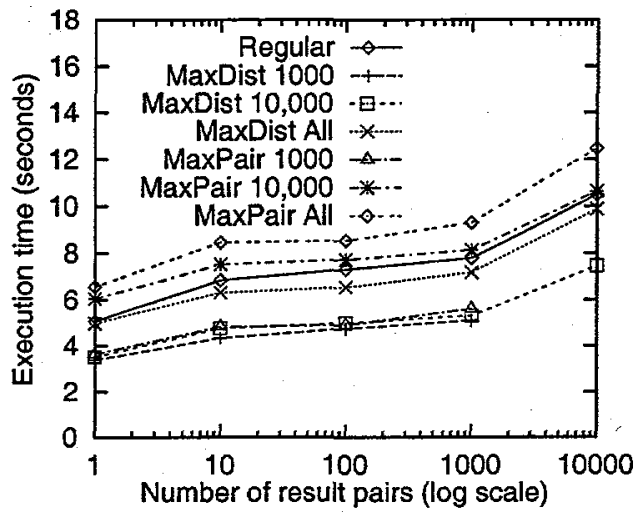

Figure 10: Execution time for different maximum distance and maximum pairs for distance semi-join.

\subsubsection{Alternative Implementations}

The distance semi-join can also be implemented using a nearest neighbor algorithm. For each object in relation $A$, we perform a nearest neighbor computation in relation $B$, and sort the resulting array of distances once all neighbors have been computed. For the data sets in question, the execution time for doing this is about 27 seconds. The incremental distance join methods reported in Figure 9 compare favorably with this method for computing the entire distance semi-join, especially "GlobalAll" (which took around 25 seconds). An even better result is obtained if we switch the order of the relations (i.e., compute the distance semi-join of Roads and Water), in which case "GlobalAll" takes about 102 seconds while the nearest neighbor implementation takes 141 seconds.

Observe that the "GlobalAll" strategy must keep track of the $d_{\max }$ distance for all objects and nodes in the R-tree for relation $A$, which can occupy considerable storage. However, an implementation that uses a nearest neighbor algorithm must also store distance values for all objects.

\section{Concluding Remarks and Directions for Fu- ture Research}

Two new spatial join operations have been defined where the join output is ordered by the distance between the spatial attribute values of the joined tuples, and a number of different incremental strategies for computing them have been examined. The rationale behind our solutions is that frequently only a small part of the join result will actually be needed. Our experiments revealed that for distance join, the variant of the incremental distance join algorithms that performed best overall was the one that processed only one node in node/node pairs at a time, attempted to traverse the two trees evenly (i.e., so as not to descend much farther into one than the other), and ordered pairs with the same distance to result in a depth-first traversal. Setting a limit on the distance of pairs was shown to improve performance considerably, even if the maximum distance limit is relatively large. However, imposing an upper bound on the number of pairs is only worthwhile if the upper bound is not very large (e.g., in our experiments, an upper bound of 100,000 pairs did not improve performance). Nevertheless, in many of the applications that we envision for our algorithm-most notably for interactive query interfaces, which quickly present the user with the most relevant part of the query result-a small upper bound can be established.

For the distance semi-join, the strategies for improving the performance of the incremental distance join were shown to yield significant improvements, especially for computing a large part of the result. The strategies use different means for eliminating from consideration pairs that are sure not to be needed to compute the output of the algorithm. The best overall strategy used every possible opportunity for eliminating pairs containing object $o_{1}$ if a pair $\left\langle o_{1}, o_{2}\right\rangle$ has been reported earlier, and uses global knowledge of distance bounds to further eliminate pairs when processing nodes ("GlobalAll" in Figure 9). This version was found to be better than a non-incremental approach that computes the distance semi-join using a nearest neighbor algorithm. However, maintaining the global knowledge of distance bounds requires a somewhat large amount of storage. A reasonable compromise is to exploit the distance bounds only locally within a node as it is being processed ("Local"). The effect of restricting the maximum distance or the maximum number of pairs was found to yield similar benefits as when computing the distance join.

Our algorithm finds use for processing queries such as "find the city nearest to any river, such that the city has a population of more than 5 million". There are at least two options for a query engine to use the incremental distance join algorithm to answer this query:

1. Execute the algorithm on the city and river relations and filter out the result pairs where the city has too small a population, and

2. First find the cities with a population greater than 5 million and use that in the incremental distance join algorithm.

For the second option, a spatial index must be built on the result of finding cities with a population of more than 5 million for the algorithm to be applicable. Hence, this option is most appropriate if the population criteria has a high selectivity. However, if the population criteria has a low selectivity, then the first option would be superior. More query plans may even exist, employing some other algorithm. To enable a query optimizer to choose between these options requires a cost model for the relevant algorithms (e.g., as developed in [20] for the traditional R-tree spatial join). Developing such cost models for the incremental distance join algorithms presented in this paper is a subject for further study.

Other issues for further investigation include developing techniques to dynamically partition the priority queue between a memory-based structure and a disk-based one. Our experiments were limited to using two-dimensional points. Further work is needed to determine how appropriate our approach is for more complex spatial objects (i.e., with extent, such as lines and polygons), as well as for higher dimensions. 


\section{Acknowledgements}

We wish to thank Björn P. Jónsson and Dr. Robert E. Webber for their critical comments.

\section{References}

[1] W. G. Aref and H. Samet. The spatial filter revisited. Proc. of 6th International Symposium on Spatial Data Handling, pp. 190-208, Edinburgh, Scotland, September 1994.

[2] F. Bartling and K. Hinrichs. Probabilistic analysis of an algorithm for solving the $k$-dimensional all-nearest-neighbors problem by projection. BIT, 31(4):558-565, 1991.

[3] R. J. Bayardo and D. P. Miranker. Processing queries for first few answers. In Proc. of 5 th CIKM, pp. 45-52, Rockville, MD, November 1996.

[4] L. Becker, K. Hinrichs, and U. Finke. A new algorithm for computing joins with grid files. Proc. of 9 th IEEE Int. Conf. on Data Engineering, pp. 190-197, Vienna, Austria, April 1993.

[5] N. Beckmann, H. P. Kriegel, R. Schneider, and B. Seeger. The $R^{*}$-tree: an efficient and robust access method for points and rectangles. Proc. of ACM SIGMOD, pp. 322-331, Atlantic City, NJ, June 1990.

[6] S. N. Bespamyatnikh. An optimal algorithm for closest pair maintenance. Proc. of 11th Symp. on Computational Geometry, pp. 152-161, Vancouver, British Columbia, June 1995.

[7] T. Brinkhoff, H. P. Kriegel, R. Schneider, and B. Seeger. Multistep processing of spatial joins. Proc. of ACM SIGMOD, pp. 197-208, Minneapolis, MN, June 1994.

[8] T. Brinkhoff, H. P. Kriegel, and B. Seeger. Efficient processing of spatial joins using R-trees. Proc. of ACM SIGMOD, pp. 237-246, Washington, DC, May 1993.

[9] Bureau of the Census. Tiger/Line precensusfiles. Washington, DC, 1989.

[10] M. J. Carey and D. Kossmann. On saying "enough already!" in SQL. Proc. of ACM SIGMOD, pp. 219-230, Tucson, AZ, May 1997.

[11] K. L. Clarkson. Fast algorithm for the all nearest neighbors problem. Proc. of 24th IEEE Symp. on the Foundations of Computer Science, pp. 226-232, Tucson, November 1983.

[12] D. Comer. The ubiquitous B-tree. ACM Computing Surveys, 11(2):121-137, June 1979.

[13] M. L. Fredman, R. Sedgewick, D. D. Sleator, and R. E. Tarjan. The pairing heap: A new form of self-adjusting heap. Algorithmica, 1(1):111-129, 1986.

[14] O. Günther. Efficient computation of spatial joins. Proc. of 9th IEEE Int. Conf. on Data Engineering, pp. 50-59, Vienna, Austria, April 1993.

[15] A. Guttman. R-trees: a dynamic index structure for spatial searching. Proc. of ACM SIGMOD, pp. 47-57, Boston, MA, June 1984.

[16] J. M. Hellerstein, P. J. Haas, and H. Wang. Online aggregation. Proc. of ACM SIGMOD, pp. 171-182, Tucson, AZ, May 1997.
[17] A. Henrich. A distance-scan algorithm for spatial access structures. Proc. of 2nd ACM Workshop on GIS, pp. 136-143, Gaithersburg, MD, December 1994.

[18] G. R. Hjaltason and H. Samet. Ranking in spatial databases. Advances in Spatial Databases - 4th Int. Symp., SSD'95, pp. 83-95, Portland, ME, August 1995. (Also Springer-Verlag Lecture Notes in Computer Science 951).

[19] E. Hoel and H. Samet. Data-parallel spatial join algorithms. Proc. of 23rd Int. Conf. on Parallel Processing, pp. 227-234, St. Charles, IL, August 1994.

[20] Y.-W. Huang, N. Jing, and E. A. Rundensteiner. A cost model for estimating the performance of spatial joins using r-trees. Proc. of 9th Int. Conf. on Scientific and Statistical Database Management, pp. 30-38, Olympia, WA, August 1997.

[21] Y.-W. Huang, N. Jing, and E. A. Rundensteiner. Spatial joins using r-trees: breadth-first traversal with global optimizations. Proc. of 23rd VLDB Conf., pp. 396-405, Athens, Greece, August 1997.

[22] M. Kitsuregawa, L. Harada, and M. Takagi. Join strategies on $k$ - $d$-tree indexed relations. Proc. of 5 th IEEE Int. Conf. on Data Engineering, pp. 85-93, Los Angeles, February 1989.

[23] D. Lomet and B. Salzberg. A robust multi-attribute search structure. Proc. of the 5th IEEE Int. Conf. on Data Engineering, pp. 296-304, Los Angeles, February 1989.

[24] D. Rotem. Spatial join indices. Proc. of 7th Int. Conf. on Data Engineering, pp. 500-509, Kobe, Japan, April 1991.

[25] N. Roussopoulos, S. Kelley, and F. Vincent. Nearest neighbor queries. Proc. of ACM SIGMOD, pp. 71-79, San Jose, CA, May 1995.

[26] H. Samet. Applications of spatial data structures: Computer graphics, image processing, and GIS. Addison-Wesley, Reading, MA, 1990.

[27] H. Samet. The design and analysis of spatial data structures. Addison-Wesley, Reading, MA, 1990.

[28] B. Seeger and H. P. Kriegel. The buddy-tree: an efficient and robust access method for spatial data base systems. Proc. of 16th VLDB Conf., pp. 590-601, Brisbane, Australia, August 1990.

[29] J. C. Shafer and R. Agrawal. Parallel algorithms for highdimensional proximity joins. Proc. of $23 r d$ VLDB Conf., pp. 176-185, Athens, Greece, August 1997.

[30] H. W. Six and D. Wood. Counting and reporting intersections of $d$-ranges. IEEE Transactions on Computers, 31(3):181187, March 1982.

[31] P. M. Vaidya. An $O(n \log n)$ algorithm for the all-nearestneighbor problem. Discrete \& Computational Geometry, 4(2):101-115, 1989.

[32] T. L. Wang and D. Shasha. Query processing for distance metrics. Proc. of I6th VLDB Conf., pp. 602-613, Brisbane, Australia, August 1990.

[33] A. N. Wilschut and P. M. G. Apers. Dataflow query execution in a parallel main-memory environment. Proc. of Ist Int. Conf. on Parallel and Distributed Information Systems, pp. 68-77, Miami, FL, December 1991. 


\title{
Speeding up Bulk-Loading of Quadtrees
}

\author{
Gísli R. Hjaltason* $\quad$ Hanan Samet* Yoram J. Sussmann ${ }^{\dagger}$ \\ Computer Science Department, Center for Automation Research, and Institute for Advanced Computer Studies, University of Maryland, \\ College Park, Maryland 20742. $\quad$ \{grh, hjs, yoram\}@es . umd. edu
}

\begin{abstract}
Spatial indexes, such as the PMR quadtree, are important in spatial databases for efficient execution of queries involving spatial constraints, especially when the queries involve spatial joins. We investigate the issue of speeding up building PMR quadtrees for a set of objects and develop two approaches to achieve this goal. In an empirical study, we find that the better method of the two offers significant improvements in execution time, and present evidence of the usefulness of spatial indexing for executing spatial join queries.
\end{abstract}

\section{Introduction}

Traditional database systems employ indexes on alphanumeric data, usually based on the B-tree, to facilitate efficient query handling. Typically, the database system allows the users to designate which attributes (data fields) need to be indexed. However, advanced query optimizers also have the ability to create indexes on unindexed data or temporary results (i.e., results from a part of the query) as needed. In order for this to be worthwhile, the index creation process must not be too time-consuming, as otherwise the operation could be executed more efficiently without an index. In other words, the index may not be particularly useful if the execution time of the operation without an index is several times faster than the total time to execute it when the time to build the index is included.

Of course, indexes are often used even though the speed of constructing them is slow when the resulting indexed data is queried many times. In this case, the time to build the index is amortized over the number of queries made on the indexed data before a new index needs to be constructed (e.g., on account of updates). This is the case when the database is static.

In the research reported here, we focus on the situation where the database is dynamic. This is an often-neglected issue in the design of spatial databases. The problem is that most often the index is chosen on the basis of the speed with which queries can be performed and on the amount of storage that is required. The queries usually involve retrieval rather than the creation of new data. This emphasis on retrieval efficiency may lead to a wrong choice of an index when the operations are not limited to retrieval. This is especially evident for a query such as the spatial join. As an example of this query, suppose that given a road relation and a river relation, we want to find all locations where a road and river meet (i.e., locations of bridges and tunnels). This can be achieved by computing a spatial join of the two relations which is realized by joining the two relations. The join condition is one that results in extracting all tuples whose spatial attribute values have at least one point in common.

The spatial join is an interesting operation because its output has both a relational and a spatial component. In practical terms, for ex-

\footnotetext{
*The support of the National Science Foundation under Grant IRI-92-16970 and the Department of Energy under Contract DEFG0295ER25237 is gratefully acknowledged.

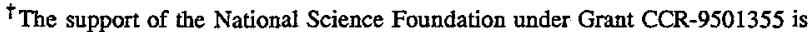
gratefully acknowledged.
}

ample in the case of line segments, we don't always want to just report the object pairs (i.e., lines or the names of the rivers and roads in our example) that intersect. In particular, we want to report their locations as well so that they can serve as input to subsequent spatial operations (i.e., a cascaded spatial join as would be common in a spatial spreadsheet). Therefore, we also need to construct a map for the output, which means that we need to construct a spatial index. In other words, the time to build the spatial index plays an important role in the overall performance of the index in addition to the time required to perform the spatial join itself whose output is not always required to be spatial. Interestingly, most traditional studies of the effect of spatial indexing on the efficiency of the spatial join (e.g., $[1,5]$ ) only focused on the relational component of the output, while very few (e.g., [6]) included a spatial component in the output.

In this paper we examine the efficiency of building the spatial index. In particular, we focus on the PMR quadtree spatial index [9]. The PMR quadtree is of particular interest as we showed in an earlier study [6] that the PMR quadtree performs quite well for a spatial join in contrast to other spatial data structures such as the R-tree (including variants such as the $\mathrm{R}^{*}$-tree) and the $\mathrm{R}^{+}$-tree.

Improving the performance of building a quadtree spatial index is of interest to us for a number of additional reasons. First of all, the PMR quadtree is used as the spatial index for the spatial attributes in a spatial database system built by us called SAND (Spatial andNonSpatial Data) [3]. SAND employs a data model inspired by the relational algebra. The basic storage unit is an attribute, which may be non-spatial (e.g., integer or character string) or spatial (e.g., points, lines, polygons, etc.). Attributes are collected into relations, and relation data is stored as tuples in tables, each of which is identified by a tuple ID. SAND uses indexing to facilitate speedy access to tuples based on both spatial and non-spatial attribute values. Second, quadtree indexes have started to appear in commercial database systems such as the Spatial Data Option (SDO) from the Oracle Corporation [10]. Thus speeding their construction has an appeal beyond our SAND prototype.

One problem with using the PMR quadtree as an index is that despite the results of our previous comparative study [6], we still find that building a PMR quadtree is a time-consuming process. Our goal is to speed up the process of building a PMR quadtree from a set of objects in order to make the PMR quadtree more useful for spatial indexing in SAND. In particular, this would make it possible for the query optimizer to build indexes on the fly as the need arises. This is especially important for queries that involve spatial joins as we saw in the example above.

We use the term bulk-loading to characterize the process of building a disk-based spatial index for an entire set of objects without any intervening queries. The approach taken in this paper is based on the idea of trying to fill up memory with as much of the quadtree as possible before writing some of its nodes on disk. Although our presentation and experiments are in terms of the PMR quadtree, our results hold for any variant of the quadtree. The rest of this paper is organized as follows. Section 2 describes the PMR quadtree and its implementation in SAND which serves as the prototype whose construction time is being speededup. Section 3 presents our approach. 
Section 4 discusses the results of our experiments, while concluding remarks are drawn in Section 5.

\section{PMR Quadtrees and their Implementation}

By the term quadtree [11] we mean a spatial data structure based on a disjoint regular decomposition of space. Each quadtree block (sometimes referred to as a cell) covers a portion of space that forms a hypercube in $d$-dimensions, usually with a side length that is a power of 2. Quadtree blocks may be further divided into $2^{d}$ subblocks of equal size. One way of conceptualizing a quadtree is to think of it as an extended $2^{d}$-ary tree ${ }^{1}$. Another way is to focus on the space decomposition, in which case it can be thought of as being an adaptive grid. Usually, there is a prescribed maximum height of the tree, or equivalently, a minimum size for each quadtree block.

The PMR quadtree is a dynamic spatial data structure based on the idea of a quadtree, where objects are stored only in leaf blocks that intersect them. If, upon inserting an object o into a quadtree block $b$, the number of objects in $b$ exceeds a splitting threshold $T$ and $b$ is not at the maximum level, then $b$ is split into $2^{d}$ sub-blocks, and the objects in $b$ (including $o$ ) are reinserted into the newly created blocks that they intersect. Note that the sub-blocks are not split further during the insertion of $o$, even if they contain more than $T$ objects. This aspect of the PMR quadtree gives rise to probabilistic behavior in the sense that the order in which the objects are inserted affects the shape of the resulting tree.

Since the PMR quadtree gives rise to a disjoint decomposition of space, and objects are stored only in leaf blocks, this implies that non-point objects may be stored in more than one leaf block. The part of an object that intersects a leaf block that contains it is often referred to as a q-object.

Quadtrees can be implemented in many different ways. One method, inspired by viewing them as trees, is to implement each block as a record, where non-leaf blocks store $2^{d}$ pointers to block records, and leaf blocks store a list of objects. However, this pointerbased approach is ill-suited for implementing disk-based structures. A general methodology for doing this is to represent only the leaf blocks in the quadtree. The location and size of each leaf block is encoded in some manner, and the result is used as a key into an auxiliary disk-based data structure. This approach is termed a linear quadtree [4].

The implementation of the PMR quadtree used in the SAND spatial database is based on a general linear quadtree implementation called the Morton Block Index (abbreviated as $M B I$ ). The size of the space covered by an MBI has side length of $2^{w}$ with 0 as the origin for each dimension, and the minimum side length of a quadtree block that can be represented is 1 . The MBI encodes quadtree blocks using a pair of numbers (termed a Morton block value): the Morton code of the quadtree block along with the side length of the block (stored in $\log _{2}$ form). The Morton code of a quadtree block is constructed by bit-interleaving the coordinate values of the lower-left corner of the block. Not all possible Morton block values correspond to legal quadtree blocks. For example, for a 2-dimensional quadtree, the only quadtree block that can have a lower-left corner of $(1,1)$ has a side length of 1 . However, each Morton code can correspond to many quadtree blocks, e.g., the point with coordinate values $(0,0)$ can be the lower-left comer of blocks of any size from 1 through $2^{w}$. Notice that the fact that the range of the Morton codes is from 0 to $2^{w}$ for each dimension is not really a limitation, as it is a simple matter to transform coordinates in any other range into the range of a Morton code, and vice versa.

Morton codes provide a mapping from a $d$-dimensional point to a one-dimensional scalar, the result of which is known as a spacefilling curve. When the $d$-dimensional points are ordered on the ba-

\footnotetext{
${ }^{2}$ An extended $k$-ary tree is a tree where each node is either a leaf node or contains $k$ children.
}

sis of their corresponding Morton codes, the order is called a Morton order. It is also known as a Z-order since it traces a ' $\mathrm{Z}$ ' pattern in two dimensions. Many other space-ordering methods exist, such as the Peano-Hilbert, Cantor-diagonal, and spiral orders. However, of those, only the Morton and Peano-Hilbert orders are useful for ordering quadtree blocks. The advantage of the Morton order is that it is much simpler, thereby making it computationally much less expensive, to convert between a Morton code and its corresponding coordinate values (and vice versa) than between a code based on Peano-Hilbert order and its corresponding coordinate values. In addition, various operations on Morton block values can be implemented through simple bit-manipulation operations on Morton codes. Examples include computing the Morton block values for sub-blocks, for a containing block as well as for the neighboring blocks of a quadtree block.

The MBI uses a B-tree to organize the Morton block values, employing a lexicographic sorting order on the Morton code and side length. Note that this corresponds to a Z-order on the quadtree blocks. For a quadtree leaf node with $k$ objects, the corresponding Morton block value is represented $k$ times in the $\mathrm{B}$-tree, once for each object. The B-tree uses a small amount of buffering of B-tree nodes, storing only the B-tree nodes from the root to the current node being searched, as well as possibly the sibling of the current node (e.g., when splitting and merging).

\section{Our Approach}

Our implementation of the PMR quadtree as described in Section 2 is very flexible in several respects. The MBI supports any number of dimensions, an underlying space with a side width of up to $2^{32}$, and the size of the object (in terms of the number of bytes) stored in the MBI is unlimited. The splitting threshold of the PMR quadtree is also unlimited. Nevertheless, we found its performance to be respectable for dynamic insertions and a wide range of queries. However, for loading a large number of objects simultaneously (i.e., bulk-loading), this flexibility proved to degrade performance. One reason for this inefficiency is that, in addition to the cost of Btree operations when traversing the tree structure implied by the quadtree, node splits are very costly. This is due to the fact that when a quadtree node is split, references to objects must be deleted from the B-tree, and then reinserted with Morton block value identifiers of the newly created quadtree nodes. The deletions from the B-tree may cause merging of B-tree nodes, and the subsequent reinsertions of the objects with their new Morton block values will then cause splitting of these same nodes. This causes a lot of disk activity.

We developed two approaches in an attempt to speed up bulkloading. One attacks the problem on the B-tree level, while the other attacks it on the PMR quadtree level. The first approach is to dramatically increase the amount of buffering done by the B-tree (the $B$-tree buffering approach). The second approach is to reduce the number of accesses to the B-tree as much as possible by storing parts of the PMR quadtree in main memory (the quadtree buffering approach).

\subsection{B-tree Buffering}

In the B-tree buffering approach, we use a buffer to store recently used B-tree nodes, and employ an LRU (least recently used) replacement policy to make space for a new node. A node locking mechanism ensures that the nodes on the path from the root to the current node are not replaced. Such extensive buffering was not included in our original implementation because it was found to offer little performance improvement for dynamic insertion as well as many query types. This is due to the fact that for such use, B-tree nodes tend to have been replaced by the time they are needed again, as quadtree blocks are requested in a largely random manner. Also, it is mostly useless for processes that access the whole quadtree in Z-order (such 
as done by some spatial join algorithm), as they give rise to a sequential scan of the MBI B-tree.

In order to make the most of the B-tree buffering approach, it is best that quadtree nodes be visited in Z-order, since that order corresponds to how they are sorted in the B-tree. By sorting the set of objects to insert in Z-order on their centroid, we will approach that goal (as they will tend to localize insertions within the top-most Btree nodes, i.e., the ones storing the largest Morton block values). Sorting a set of objects prior to insertion is a small price to pay, as it is usually a much less expensive process than the cost of building the spatial index. It is a common approach for statically built spatial data structures (e.g., Hilbert-packed R-trees [7]).

\subsection{Quadtree Buffering}

In the quadtree buffering approach, we build a pointer-based quadtree in main memory, thereby bypassing the MBI B-tree. Of course, this can only be done as long as the entire quadtree fits in main memory. Once available memory is used up, parts of the pointer-based quadtree are flushed (i.e., written) onto disk (i.e., into the MBI). When all the objects have been inserted into the pointerbased quadtree, the entire tree is inserted into the MBI. In order to maintain compatibility with the MBI-based PMR structure, we use Morton block values to determine the space coverage of quadtree blocks. Note that it is not necessary to store the Morton block values in the nodes of the pointer-based structure (each node corresponds to a quadtree block), as these can be computed during traversals of the tree. However, a careful analysis of execution profiles revealed that a large percentage of the execution time was spent on bitmanipulation operations on Morton block values. Thus, we chose to store the Morton block values in the nodes, even though this increased their storage requirements.

We use a set of heuristics to choose which quadtree blocks (also referred to as nodes) to flush. The goal is to flush quadtree blocks that will not be needed later on, i.e., no subsequently inserted object intersects the block. In general, it is impossible to attain this goal for arbitrary insertion patterns. However, sorting the set of objects prior to building the spatial index makes it possible to get close to the goal. As in the case of the B-tree buffer approach, sorting the objects in Z-order does the trick. Using such an ordering would mean that the insertion activity would be rather localized in the tree, so once nothing has been inserted into a node $a$ for a long time, $a$ will most probably not be inserted into again.

The process of choosing quadtree blocks to flush makes use of a set of statistics that is maintained for each node $a$ :

1. The time at which the last insertion was made into the quadtree block corresponding to $a$. ,

$\checkmark$ 2. The number of q-objects in the subtree rooted at $a$ (recall that a q-object is the part of an object that intersects a containing leaf block).

3. The number of nodes in the subtree rooted at $a$, not counting the node $a$ itself (for leaf nodes, this is always 0 ).

After a node in the pointer-based quadtree is flushed to the MBI, its contents (i.e., child nodes for non-leaf nodes or object lists for leaf nodes) are deallocated, and the node is marked as an MBI node. The node is kept around in case there are subsequent insertions into it, in which case the MBI-based PMR quadtree insertion routine is invoked for that quadtree block. The amount of memory used by a subtree is proportional to the number of q-objects and nodes in the subtree. Thus, the flushing process is guaranteed to free a certain percentage $Q$, termed the flushing quotient, of the q-objects or nodes. The decision process is recursive on the pointer-based quadtree nodes and starts at the root. Initially, the number of qobjects, $N_{q}$, and nodes, $N_{n}$, to free is set at $Q$ times the total number of q-objects and nodes in the memory-based structure. Each time a node is flushed, $N_{q}$ and $N_{n}$ are reduced as appropriate, terminating the process if either value reaches zero (actually, $N_{n}$ never reaches zero unless it is a multiple of $2^{d}$, so either it can be made a multiple of $2^{d}$ initially, or the terminating condition can be made that it is less than $2^{d}$ ).

A recursive invocation of the flushing method proceeds as follows, where $n$ is the node under consideration. If $n$ is a leaf node, it is flushed. For a non-leaf node, if the number of $q$-objects and nodes in the subtree rooted at $n$ is less than or equal to $N_{q}$ and $N_{n}$, respectively, then the whole subtree rooted at $n$ is flushed to the MBI. Otherwise, the child nodes of $n$ are considered in the order of their last insertion time (i.e., nodes that have not been inserted into the longest are considered first). The flushing process is applied recursively to the child nodes that have not yet been flushed and whose number of $\mathrm{q}$-objects is at least $\frac{1}{2^{a}}$ times the number of q-objects in the whole subtree rooted at $n$ (recall that $n$ has $2^{d}$ child nodes implying that this quantity is the average number of q-objects in the child nodes). The latter rule is a heuristic that tends to flush nodes that will not be inserted into again, given that objects are inserted in $\mathrm{Z}$-order, as it makes sure that only child nodes with an above average number of q-objects are processed. Note that often, only the first few child nodes of $n$ are looked at, since the process terminates once enough q-objects and nodes have been freed from memory.

In addition to controlling how much memory is freed, the flushing quotient provides a means for controlling how deep into the tree the recursive flushing process descends in its search for nodes to flush. In other words, a small flushing quotient will cause a deeper descent into the tree than will a large flushing quotient. Also, note that we don't require both $N_{q}$ and $N_{n}$ to fall down to zero. The reason is that this tends to cause too many nodes to be flushed (i.e., objects are highly likely to be inserted into many of the flushed nodes). The reason for basing the heuristic process on both the number of q-objects and nodes is that it provides more consistent results (in terms of the amount of memory that is freed) than using only one, especially for small buffer sizes.

\section{Empirical Results}

We implemented both of our approaches to speeding up the bulkloading of quadtrees and ran experiments with two-dimensional line data, both real-world and randomly generated. The real-world data consists of three data sets from the TIGER/Line File [2]:

1. Washington DC: 19,185 line segments.

2. Prince George's County, MD: 59,551 line segments.

3. Roads in Washington DC metro area: 200,482 line segments.

The randomly generated data sets have $64,000,128,000$ and 256,000 line segments and were constructed by generating random infinite lines in a manner that is independent of translation and scaling of the coordinate system [8]. These lines are clipped to the map area to obtain line segments, and then subdivided further at intersection points with other line segments so that at the end, line segments meet only at endpoints.

In our experiments, we chose to store the entire geometry of the objects in the PMR quadtree. The side length of the space containing the data was $2^{15}=32768$ and the splitting threshold was set at 8. Larger splitting thresholds make the quadtree buffering approach even more attractive. However, as 8 is a commonly used splitting threshold, this is the value we used.

The programs we used were compiled with the GNU $\mathrm{C}++$ compiler with full optimization (-O3), and the experiments were conducted on a SUN SPARCstation 5 Model 70 (rated at 60 SPECint 92 and 47 SPECfp92) with $32 \mathrm{MB}$ of memory. 
Figure 1 shows the speedup in the insertion time for five buffering methods when the line segments are inserted in Z-order (including the time for sorting the line segments) in comparison to inserting the segments in their original order without buffering. In the figure, "BB-large" and "BB-small" denote B-tree buffering with a large buffer and a small buffer ( 100 nodes occupying $400 \mathrm{~K}$ ), respectively. The large buffer size fills almost all available memory, and is large enough to hold the entire quadtree except for the two largest data sets. Similarly, "QB-large" and "QB-small" denote quadtree buffering with a large and a small buffer (100K), respectively. Again, the large buffer for quadtree buffering is large enough to hold entire quadtree for all but the largest data sets. The reason for including the cases using the large buffers is to reveal the maximum speedup that can be achieved with buffering, as in this extreme case no flushing of quadtree nodes needs to be done until the whole tree has been built. This gives a useful yardstick for assessing the performance of our flushing heuristics when using a small buffer. While $100 \mathrm{~K}$ may not seem like a very small buffer, it is nevertheless a small fraction of the size of the index, or less than $4 \%$ for the DC data set, and even less for the others. Furthermore, in an era when $32 \mathrm{MB}$ of main memory is considered small, $100 \mathrm{~K}$ is not very "large". Finally, "Both-small" is the result of using both buffering methods simultaneously with a small buffer size for both. We used a flushing quotient of .25 for the quadtree buffering method.

The most startling observation we can make from Figure 1 is that quadtree buffering is up to more than 8 times faster than not using any buffering when the quadtree buffers are large, while being about 5 times faster when the quadtree buffers are small.

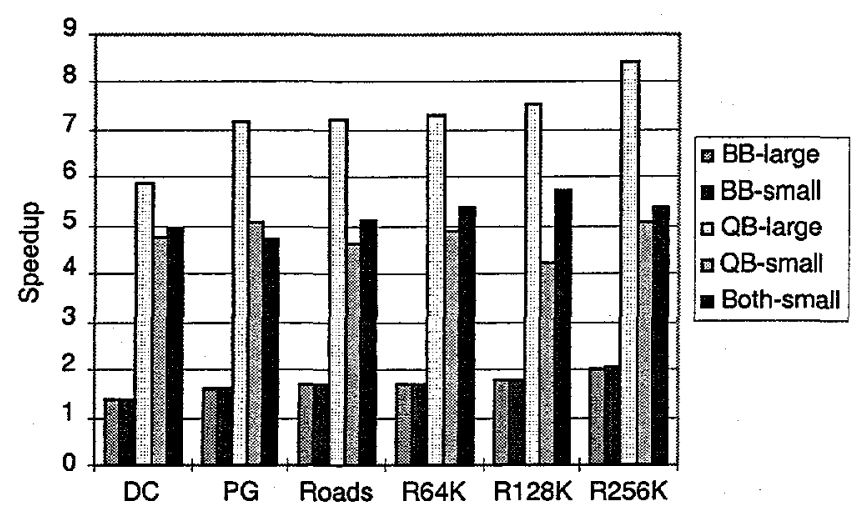

Figure 1: Improvements of the buffering methods over no buffering, taking sorting time into account.

\section{Concluding Remarks}

We have shown that quadtree buffering enabled us to build a quadtree in time that was an order of magnitude smaller than our original PMR quadtree implementation. Even when using modest amounts of memory for the buffering, the improvement was considerable (a factor of 5). The B-tree buffering approach offered some improvements over the original implementation that did not use any buffering, but not nearly as much as the quadtree buffering approach, even for large buffer capacity when disk activity is kept at a minimum. This demonstrates that the linear quadtree storage method is highly CPU intensive, at least for insertions. A significant portion of the CPU time is spent in computing operations on Morton block values, a cost that was avoided in the quadtree buffering approach by storing the Morton block values in the pointerbased quadtree structure. Other factors that explain the difference in performance are the higher overhead involved in traversing the quadtree through the MBI as well as the repeated splitting and merging of B-tree nodes resulting from quadtree node splits due to deletion and reinsertions of Morton block values.

Future work includes investigating whether our buffering strategies for bulk-loading may be used to speed up dynamic insertions and queries. Also, the fact that our system can build PMR quadtrees efficiently will enable us to build a spatial query processor for SAND that exploits this to construct spatial indexes for temporary results (e.g., results from other, possibly non-spatial, queries), or for un-indexed spatial relations, prior to spatial operations on them. This is particularly important for complex operations such as spatial joins.

\section{References}

[1] T. Brinkhoff, H. P. Kriegel, and B. Seeger. Efficient processing of spatial joins using R-trees. In Proceedings of the 1993 ACM SIGMOD International Conference, pages 237-246, Washington, DC, May 1993.

[2] Bureau of the Census. Tiger/Line precensusfiles. Washington, DC, 1989.

[3] C. Esperança and H. Samet. An overview of the SAND spatial database system. Communications of the $A C M$, to appear 1997.

[4] I. Gargantini. An effective way to represent quadtrees. Communications of the ACM, 25(12):905-910, December 1982.

[5] O. Günther. Efficient computation of spatial joins. In Proceedings of the 9th IEEE International Conference on Data Engineering, pages 50-59, Vienna, Austria, April 1993.

[6] E. G. Hoel and H. Samet. Benchmarking spatial join operations with spatial output. In U. Dayal, P. M. D. Gray, and S. Nishio, editors, Proceedings of the 21st International Conference on Very Large Data Bases, pages 606-618, Zurich, September 1995.

[7] I. Kamel and C. Faloutsos. On packing R-trees. Second International Conference on Information and Knowledge Management (CIKM), pages 490-499, November 1993.

[8] M. Lindenbaum and H. Samet. A probabilistic analysis of triebased sorting of large collections of line segments. Computer Science Department TR-3455, University of Maryland, College Park, MD, April 1995.

[9] R. C. Nelson and H. Samet. A population analysis for hierarchical data structures. In Proceedings of the SIGMOD Conference, pages 270-277, San Francisco, May 1987.

[10] Oracle Corporation. Advances in relational database technology for spatial data management. Oracle spatial data option technical white paper, September 1996.

[11] H. Samet. The Design and Analysis of Spatial Data Structures. Addison-Wesley, Reading, MA, 1990. 


\title{
Distance Browsing in Spatial Databases ${ }^{1}$
}

\author{
Gísli R. Hjaltason and Hanan Samet \\ Computer Science Department \\ Center for Automation Research \\ Institute for Advanced Computer Studies \\ University of Maryland \\ College Park, Maryland 20742 \\ grh@cs.umd.edu and hjs@cs.umd.edu
}

\begin{abstract}
Two different techniques of browsing through a collection of spatial objects stored in an R-tree spatial data structure on the basis of their distances from an arbitrary spatial query object are compared. The conventional approach is one that makes use of a $k$-nearest neighbor algorithm where $k$ is known prior to the invocation of the algorithm. Thus if $m>k$ neighbors are needed, the $k$-nearest neighbor algorithm needs to be reinvoked for $m$ neighbors, thereby possibly performing some redundant computations. The second approach is incremental in the sense that having obtained the $k$ nearest neighbors, the $k+1^{\text {st }}$ neighbor can be obtained without having to calculate the $k+1$ nearest neighbors from scratch. The incremental approach finds use when processing complex queries where one of the conditions involves spatial proximity (e.g., the nearest city to Chicago with population greater than a million), in which case a query engine can make use of a pipelined strategy. A general incremental nearest neighbor algorithm is presented that is applicable to a large class of hierarchical spatial data structures. This algorithm is adapted to the R-tree and its performance is compared to an existing $k$-nearest neighbor algorithm for R-trees [45]. Experiments show that the incremental nearest neighbor algorithm significantly outperforms the $k$-nearest neighbor algorithm for distance browsing queries in a spatial database that uses the R-tree as a spatial index. Moreover, the incremental nearest neighbor algorithm also usually outperforms the $k$-nearest neighbor algorithm when applied to the $k$-nearest neighbor problem for the R-tree, although the improvement is not nearly as large as for distance browsing queries.
\end{abstract}

Keywords: distance browsing, ranking, nearest neighbors, R-trees, spatial databases, hierarchical spatial data structures.

This is a preliminary release of an article accepted by ACM Transactions on Database Systems. The definitive version is currently in production at ACM and, when released, will supersede this version.

\footnotetext{
${ }^{1}$ This work was supported in part by the National Science Foundation under grants IRI-9712715 and the Department of Energy under Contract DEFG0295ER25237.
} 


\section{Introduction}

In this paper, we focus on the issue of obtaining data objects in their order of distance from a given query object (termed ranking). This issue is of primary interest in a spatial database although it also finds use in other database applications including multimedia indexing [36], CAD, and molecular biology [37]. The desired ranking may be full or partial (e.g., only the first $k$ objects). This problem can also be posed in a conventional database system. For example, given a table of individuals containing a weight attribute, we can ask "who has a weight closest to $w$ lbs.?", or "rank the individuals by how much their weight differs from $w$ lbs.". If no index exists on the weight attribute, then to answer the first query, a scan of all tuples must be performed. However, if an appropriate index structure is used, then more efficient methods can be employed. For example, using a $\mathrm{B}^{+}$-tree, the query can be answered by a single descent to a leaf, for a cost of $O(\log n)$ for $n$ tuples. The correct answer will be found either in that leaf or an adjacent one. To rank all the individuals, the search would proceed in two directions along the leaves of the $\mathrm{B}^{+}$-tree, with a constant cost for each tuple. The index can be used for any such query regardless of the reference weight $w$.

For multidimensional data, things are not so simple. Consider, for example, a set of points in two dimensions representing cities. Queries analogous to the previous ones are "what city is closest to point $p$ ?" and "rank the cities by their distances from point $p$ ". In a database context, we wish to know what kind of index structures will aid in processing these queries. For a fixed reference point $p$ and distance metric, we might build a one-dimensional index on the distances of the cities from the point $p$. This would provide an efficient execution time for this particular point (i.e., for $p$ ), but for any other point or distance metric it would be useless. Thus we have to rebuild the index, which is a costly process if we need to do it for each query. Contrast this to the one-dimensional case, where there is generally only one choice of metric. Furthermore, for a given reference point, any other point can have only two positions in relation to it, larger or smaller. It is not possible to define such a simple relationship in the multidimensional case.

As another example, suppose we want to find the nearest city to Chicago that has more than a million inhabitants. There are several ways to proceed. An intuitive solution is to guess some area range around Chicago and check the populations of the cities in the range. If we find a city with the requisite population, we must make sure that there are no other cities that are closer and that meet the population condition. This approach is rather inefficient as we have to guess the size of the area to be searched. The problem with guessing is that we may choose too small a region or too large a region. If the size is too small, the area may not contain any cities satisfying the population criterion, in which case we need to expand the region being searched. If the size is too large, we may be examining many cities needlessly.

A radical solution is to sort all the cities by their distances from Chicago. This is not very practical as we need to re-sort them each time we pose a similar query with respect to another city. Moreover, sorting requires a considerable amount of extra work, especially when usually all that is needed to obtain the desired result is to inspect the first few nearest neighbors.

A less radical solution is to retrieve the closest $k$ cities and determine if any of them satisfy the population criterion. The problem here lies in determining the value of $k$. As in the area range solution, we may choose too small or too large a value of $k$. If $k$ is too small, failure to find a city satisfying the population criterion means that we have to restart the search with a value larger than $k$, say $m$. The drawback of this solution is that such a search forces us to expend work in finding the $k$ nearest neighbors (which we already did once before) as part of the cost of finding the $m>k$ nearest neighbors. On the other hand, if $k$ is too large, we waste work in calculating neighbors whose populations we will never check.

A logical way to overcome the drawbacks of the second and third solutions is to obtain the neighbors 
incrementally (i.e., one by one) as they are needed. In essence, what we are doing is browsing through the database on the basis of distance and we shall use the term distance browsing to describe this operation. The result is an incremental ranking of the cities by distance where we cease the search as soon as the secondary population condition is satisfied. The idea is that we want only a small but unknown number of neighbors. The incremental solution finds application in a much more general setting than our specialized query example. In particular, this includes queries that require the application of the "nearest" function to a subset $s$ of the attributes of a relation (or object class) $r$. This class of queries is part of a more restricted, but very common, class that imposes an additional condition $c$ usually involving attributes other than $s$. This means that the "nearest" condition serves as a primary condition, while condition $c$ serves as a secondary condition. Using an incremental solution enables such a query to be processed in a pipelined fashion.

Of course, in the worst case, we will have to examine all (or most) of the neighbors even when using an incremental approach. This may occur if few objects satisfy the secondary condition (e.g., if none of the cities have the requisite population). In this case, it may actually be better to first select on the basis of the secondary condition (the population criterion in our example) before considering the "spatially nearest" condition, especially if an index exists that can be used to compute the secondary condition. Using a $k$-nearest neighbor algorithm may also be preferable, provided it is more efficient than the incremental algorithm for large values of $k$. It makes sense to choose this solution only if we know in advance how many neighbors are needed (i.e., the value of $k$ ), but this value can be estimated based on the selectivity of the secondary condition. These issues demonstrate the need for a query engine to make estimates using selectivity factors (e.g., $[3,40,49]$ ) involving the numbers of values that are expected to satisfy various parts of the query and the computational costs of the applicable algorithms.

In this paper we compare the incremental and $k$-nearest neighbor approaches for browsing through a collection of spatial objects stored in an R-tree spatial data structure on the basis of their distances from an arbitrary spatial query object. In the process we present a general incremental nearest neighbor algorithm that is applicable to a large class of hierarchical spatial data structures, and show how to adapt this algorithm to the R-tree. Its performance is compared to an existing $k$-nearest neighbor algorithm for R-trees [45]. In addition, we demonstrate that the $k$-nearest neighbor algorithm of [45] can be transformed into a special case of our R-tree adaptation of the general incremental nearest neighbor algorithm. The transformation process also reveals that the R-tree incremental nearest neighbor algorithm achieves more pruning than the R-tree $k$-nearest neighbor algorithm. Moreover, our R-tree adaptation leads to a considerably more efficient (and conceptually different) algorithm. This is because the presence of object bounding rectangles in the tree enables their use as pruning devices to reduce disk $\mathrm{I} / \mathrm{O}$ for accessing the spatial descriptions of objects (stored external to the tree). Experiments show that the incremental nearest neighbor algorithm significantly outperforms the $k$-nearest neighbor algorithm for distance browsing queries in a spatial database that uses the $\mathrm{R}$-tree as a spatial index. Moreover, the incremental nearest neighbor algorithm also usually outperforms the $k$-nearest neighbor algorithm when applied to the $k$-nearest neighbor problem for the $\mathrm{R}$-tree, although the improvement is not nearly as large as for distance browsing queries.

The rest of this paper is organized as follows. Section 2 discusses algorithms related to nearest neighbor queries. Section 3 reviews the structure of R-trees. Section 4 describes the incremental nearest neighbor algorithm as well as its adaptation to the $\mathrm{R}$-tree. Section 5 introduces the $k$-nearest neighbor algorithm. Section 6 presents the results of an empirical study comparing the incremental nearest neighbor algorithm with the $k$-nearest neighbor algorithm. Section 7 discusses issues that arise in high-dimensional spaces, while conclusions are drawn in Section 8. 


\section{Related Work}

Numerous algorithms exist for answering nearest neighbor and $k$-nearest neighbor queries. This is motivated by the importance of these queries in fields including geographical information systems (GIS), pattern recognition, document retrieval, and learning theory. Almost all of these algorithms, many of them coming from the field of computational geometry, are for points in a $d$-dimensional vector space $[12,16,21,22,33,45,51]$, but some allow for arbitrary spatial objects [26,30], although most are still limited to a point as the query object. In many applications, a rough answer suffices, so that algorithms have been developed that return an approximate result $[4,10,54]$, thereby saving time in computing it. Many of the above algorithms require specialized search structures $[4,10,16,22,33]$, but some employ commonly used spatial data structures. For example, algorithms exist for the $\mathrm{k}-\mathrm{d}$ tree $[12,21,41,51]$, quadtree-related structures $[29,30]$, the Rtree [45, 54], the LSD-tree [26] and others. In addition, many of the algorithms can be applied to other spatial data structures.

To our knowledge, only three incremental solutions to the nearest neighbor problem exist in the literature $[12,26,29]$. All these algorithms employ priority queues (see Section 4). The algorithm of [12] was developed for a $\mathrm{k}$-d tree [7]. It is considerably different from the other two algorithms in that the algorithm of [12] stores only the data objects in the priority queue, and uses a stack to keep track of the subtrees of the spatial data structure which have yet to be completely processed. This makes it necessary to use an elaborate mechanism to avoid processing the contents of a node more than once. The algorithm of [26] was developed for the LSD-tree [28]. It is very similar to our method (presented in [29]) and was published at about the same time. The principal difference between [26] and our method is that the LSD-tree algorithm uses two priority queues, one for the data objects and another for the nodes of the spatial data structure. This makes the algorithm somewhat more complicated than ours, while the use of two priority queues does not offer any performance benefits according to our experiments. Our algorithm [29] was initially developed for the PMR quadtree [42] although its presentation was general. In this paper we expand considerably on our initial solution by showing how it can be adapted to the R-tree [24] as well as comparing it with a solution that makes use of an existing $k$-nearest neighbor algorithm [45]. In addition, we show how this $k$-nearest neighbor algorithm [45] can be transformed into a special case of our R-tree adaptation of the general incremental nearest neighbor algorithm. A byproduct of the transformation process is that the $k$-nearest neighbor algorithm has been simplified considerably.

The term distance scan [5] has also been used for what we term distance browsing. Becker and Güting [5] introduce the concept of a distance scan and motivate its use. This is done along similar lines to those of Section 1 , i.e., in the context of finding the closest object to a query point where additional conditions may be imposed on the object. In addition, that paper provides optimization rules for mapping a "closest" operator into a "distance scan" operation in an example GIS query language.

All the algorithms mentioned thus far assume that the objects exist in a $d$-dimensional Euclidean space, so that distances are defined between every two objects in a data set as well as between an object and any point in the space. Another class of nearest neighbor algorithms operates on more general objects, in what is commonly called the metric space model. The only restriction on the objects is that they reside in some metric space, i.e., a distance is defined between any two objects. However, in this general case, it is not possible to produce new objects in the metric space, e.g., to aggregate or divide two objects (in a Euclidean space, bounding rectangles are often used for this purpose). Various methods exist for indexing objects in the metric space model as well as for computing proximity queries $[11,13,14,52,53]$. These methods can only make use of the properties of distance functions (non-negativity, symmetry, and the triangle inequality), and operate without any knowledge of how objects are represented or how the distances between objects are computed. Such a general approach is usually slower than methods based on spatial properties of objects, 
but must be used for objects for which such properties do not exist (e.g., images, chemical data, time series, etc.). This approach has also been advocated for high-dimensional spaces. It may often be possible to map general objects into geometric space, thereby reaping the benefit of more efficient search methods. Most such mapping approaches are domain-specific [25,36], but general approaches have also been proposed [18].

\section{R-trees}

The R-tree (e.g., Figure 1) [24] is an object hierarchy in the form of a balanced structure inspired by the $\mathrm{B}^{+}$. tree [15]. Each R-tree node contains an array of (key, pointer) entries where key is a hyper-rectangle that minimally bounds the data objects in the subtree pointed at by pointer. In an R-tree leaf node, the pointer is an object identifier (e.g., a tuple ID in a relational system), while in a non-leaf node it is a pointer to a child node on the next lower level. The maximum number of entries in each node is termed its node capacity or fan-out and may be different for leaf and non-leaf nodes. The node capacity is usually chosen such that a node fills up one disk page (or a small number of them). It should be clear that the R-tree can be used to index a space of arbitrary dimension and arbitrary spatial objects rather than just points.

As described above, an R-tree leaf node contains a minimal bounding rectangle and an object identifier for each object in the node, i.e., the geometric descriptions of the objects are stored external to the R-tree itself. Another possibility is to store the actual object, or its geometric description, in the leaf instead of its bounding rectangle. This is usually useful only if the object representation is relatively small (e.g., similar in size to a bounding rectangle) and is fixed in length. If all the data about the object (i.e., all its relevant attributes) are stored in the leaf nodes, the object identifiers need not be stored. The disadvantage of this approach is that objects will not have fixed addresses, as some objects must be moved each time an R-tree node is split.

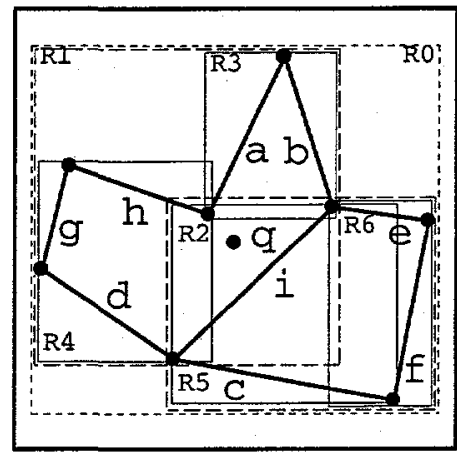

(a)

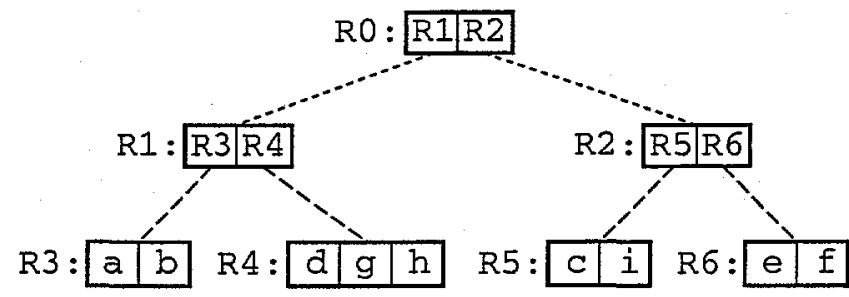

(b)

Figure 1: An R-tree index for a set of nine line segments. (a) Spatial rendering of the line segments and bounding rectangles; (b) a tree access structure for (a). The bounding rectangles for the individual line segments are omitted from (a) in the interest of clarity.

Several variations of R-trees have been devised, differing in the way nodes are split or combined during insertion or deletion. In our experiments we make use of a variant called the $\mathrm{R}^{*}$-tree [6]. It differs from the conventional R-tree in employing more sophisticated insertion and node-splitting algorithms that attempt to minimize a combination of overlap between bounding rectangles and their total area. In addition, when $\mathrm{R}$ tree node $p$ overflows, instead of immediately splitting $p$, the $\mathrm{R}^{*}$-tree insertion algorithm first tries to see if some of the entries in $p$ could possibly fit better in another node. This is achieved by reinserting a fixed 
fraction of the entries in $p$. This increases the construction time for the index, but usually results in less node overlap and therefore in improved query response time.

\section{Incremental Nearest Neighbor Algorithm}

Most algorithms that traverse tree structures in a top-down manner use some form of depth-first or breadthfirst tree traversal. Finding a leaf node containing a query object $q$ in a spatial index can be done in a depthfirst manner by recursively descending the tree structure. With this method, the recursion stack keeps track of what nodes have yet to be visited. Having reached a leaf, we need to be able to extend this technique to find the nearest object, as the leaf may not actually contain the nearest neighbor. The problem here is that we have to unwind the recursion to find the nearest object. Moreover, if we want to find the second nearest object, the solution becomes even tougher. With breadth-first traversal, the nodes of the tree are visited level by level, and a queue is used to keep track of nodes that have yet to be visited. However, with this technique, a lot of work has to be done before reaching a leaf node containing $q$. To resolve the problems with depth-first and breadth-first traversal, the incremental nearest neighbor algorithm employs what may be termed best-first traversal. When deciding what node to traverse next, it picks the node with the least distance in the set of all nodes that have yet to be visited. This means that instead of using a stack or a plain queue to keep track of the nodes to be visited, we use a priority queue where the distance from the query object is used as a key. The key feature of our solution is that the objects as well as the nodes are stored in the priority queue.

This section is organized as follows: In Section 4.1 we specify what conditions must hold for our incremental nearest neighbor algorithm to be applicable (e.g., conditions on the index, spatial object types, distance functions, etc.). In Section 4.2 we present the general incremental nearest neighbor algorithm in detail. In Section 4.3 we discuss ways to exploit the particular nature of the R-tree spatial index, while in Section 4.4 we give an example of the execution of the algorithm on a simple R-tree structure. Several variants of the algorithm are described in Section 4.5. In Section 4.6 we present some analytical results for the algorithm, while in Section 4.7 we prove its correctness. Finally, in Section 4.8 we show how to deal with a large priority queue.

\subsection{Introduction}

Our incremental nearest neighbor algorithm can be applied to virtually any hierarchical spatial data structure. In fact, it is generally applicable to any data structure based on hierarchical containment/partitioning (e.g., see [1]). In our description, we will assume a tree structure (although our method is applicable to more general structures), where each tree node represents some regions of space and where objects (or pointers to them in an external table) are stored in the leaf nodes whose regions intersect the objects. In the remainder of this section, we do not make a distinction between a node and the region that it represents; the meaning should be clear from the context. A basic requirement for the method to be applicable is that the region covered by a node must be completely contained within the region(s) of the parent node(s) ${ }^{1}$. Examples of structures that satisfy this requirement include quadtrees [47], R-trees [24], $\mathrm{R}^{+}$-trees [50], LSD-trees [28], and k-d-Btrees [44]. In all these examples, the node region is rectangular, but this is not a requirement. Our algorithm handles the possibility of an object being represented in more than one leaf node, as in the PMR quadtree [42] and $\mathbf{R}^{+}$-tree [50]. Although we assume in our exposition that each node has only one parent and that only

\footnotetext{
${ }^{1}$ For structures in which each node can have more than one parent (e.g., the hB-tree [39] or Partition Fieldtree [19]) the node region must be fully contained in the union of the regions of the parent nodes.
} 
leaf nodes store objects, the algorithm could easily be adapted to handle other cases (such as the hB-tree [39] and the cell tree with oversize shelves [23]).

Observe that the data objects as well as the query objects can be of arbitrary type (e.g., points, rectangles, polygons, etc.). The only requirement is that consistent distance functions $d_{o}$ and $d_{n}$ be used for calculating the distance from the query object $q$ to data objects and to nodes. This is to ensure that each object is encountered in at least one node that is no farther from the query object than the object itself; otherwise, the strictly non-decreasing distances of elements retrieved from the queue cannot be guaranteed. Consistency can be defined formally as follows: (In the definition, we do not make any assumptions about the nature of the index hierarchy.)

Definition Let $d$ be the combination of functions $d_{o}$ and $d_{n}$, and let $e \sqsubseteq N$ denote the fact that item $e$ is contained in exactly set of nodes $N$ (i.e., if $e$ is an object, $N$ is the set of leaf nodes referencing the object, and if $e$ is a node, $N$ is its set of parent nodes ${ }^{2}$ ). The functions $d_{\circ}$ and $d_{n}$ are consistent iff for any query object $q$ and any object or node $e$ in the hierarchical data structure there exists $n$ in $N$, where $e \sqsubseteq N$, such that $d(q, n) \leq d(q, e)$.

This definition is strictly tied to the hierarchy defined by the data structure. However, since this hierarchy is influenced by properties of the node regions and data objects, we can usually recast the definition in terms of these properties. For example, in spatial data structures the containment of objects in leaf nodes and child nodes in parent nodes is based on spatial containment; thus the $\sqsubseteq$ in the definition also denotes spatial containment. In other words, $e \sqsubseteq N$ means that the union of the node regions for the nodes in $N$ completely encloses the region covered by the object or node $e$. Informally, our definition of consistency means that if $p$ is the point in $e$ (or, more accurately, in the region that corresponds to it) closest to $q$, then $p$ must also be contained in the region covered by some node in $N$. Note that since we assume spatial indexes that form a tree hierarchy (i.e., each non-root node has exactly one parent), in the case of nodes the definition above simplifies to the following condition: if $n^{\prime}$ is a child node of node $n$, then $d_{n}(q, n) \leq d_{n}\left(q, n^{\prime}\right)$.

An easy way to ensure consistency is to base both functions on the same metric $d_{p}\left(p_{1}, p_{2}\right)$ for points; common choices of metrics include the Euclidean, Manhattan and Chessboard metrics. We then define $d(q, e):=$ $\min _{p_{1} \in q, p_{2} \in e} d_{p}\left(p_{1}, p_{2}\right)$, where $e$ is either a spatial object or a node region. It is important to note that this is not the only way to define consistent distance functions. When $d$ is defined based on a metric $d_{p}$, its consistency is guaranteed by the properties of $d_{p}$, specifically, non-negativity and the triangle inequality. The non-negativity property states, among other things, that $d_{p}(p, p)=0$, and the triangle inequality states that $d_{p}\left(p_{1}, p_{3}\right) \leq d_{p}\left(p_{1}, p_{2}\right)+d_{p}\left(p_{2}, p_{3}\right)$. Since $e$ is spatially contained in $N, e$ and $N$ have points in common, so their distance is zero. Thus, according to the triangle inequality, $d(q, e) \leq d(q, N)+d(N, e)=d(q, N)$, using a broad definition of $d$ (to allow $d(N, e)$, which equals 0 ). Note that if the distance functions are defined in this way, the distance from a query object to a node that intersects it is zero (i.e., it is not equal to the distance to the boundary of the node region).

The incremental nearest neighbor algorithm works in any number of dimensions, although the examples we give are restricted to two dimensions. Also, the query object need not be in the space of the dataset.

\subsection{Algorithm Description}

We first consider a regular recursive top-down traversal of the index to locate a leaf node containing the query object. Note that there may be more than one such node. The traversal is initiated with the root node of the

\footnotetext{
${ }^{2}$ In most spatial data structures, each node has only one parent node; the $h B$-tree is an exception.
} 
spatial index (i.e., the node spanning the whole index space) as the second argument.

\section{FINDLEAF(QueryObject, Node)}

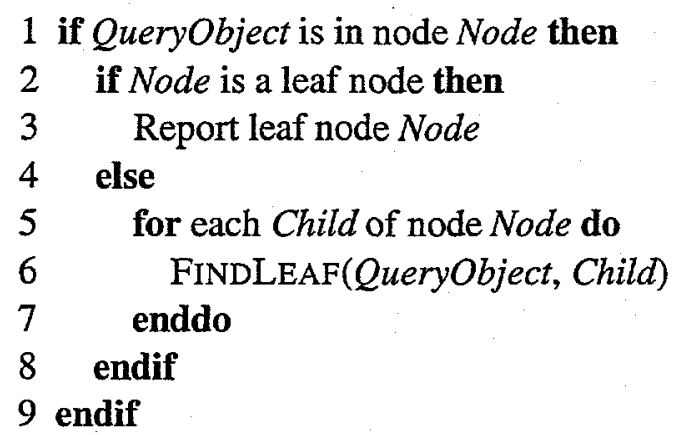

The first task is to extend the algorithm to find the object nearest to the query object. In particular, once a leaf node containing QueryObject has been found in line 3, we could start by examining the objects contained in that node. However, the object closest to the query object might reside in another node. Finding that node may in fact require unwinding the recursion to the top and descending again deeper into the tree. Furthermore, once that node has been found, it does not aid in finding the next nearest object.

To resolve this dilemma, we replace the recursion stack of the regular top-down traversal with a priority queue. In addition to using the priority queue for nodes, objects are also put on the queue as leaf nodes are processed. The key used to order the elements on the queue is distance from the query object. In order to distinguish between two elements at equal distances from the query object, we adopt the convention that nodes are ordered before objects, while objects are ordered according to some arbitrary (but unique) rule. This secondary ordering makes it possible to avoid reporting an object more than once, which is necessary when using a disjoint decomposition, e.g., a PMR quadtree [42] or an $\mathrm{R}^{+}$-tree [50], in which non-point objects may be associated with more than one node.

A node is not examined until it reaches the head of the queue. At this time, all nodes and objects closer to the query object have been examined. Initially, the node spanning the whole index space is the sole element in the priority queue. At subsequent steps, the element at the head of the queue (i.e., the closest element not yet examined) is retrieved, and this is repeated until the queue has been emptied. Informally, we can visualize the progress of the algorithm for a query object $q$ as follows, when $q$ is a point (see Figure 2). We start by locating the leaf node(s) containing $q$. Next, imagine a circle centered at $q$ being expanded from a starting radius of 0 ; we call this circle the search region. Each time the circle hits the boundary of a node region, the contents of that node are put on the queue, and each time the circle hits an object, we have found the object next nearest to $q$. Note that when the circle hits a node or an object, we are guaranteed that the node or object is already in the priority queue, since the node that contains it must already have been hit (this is guaranteed by the consistency condition).

Figure 3 presents the algorithm. Lines 1-2 initialize the queue. Notice that it is not really necessary to provide the correct distance when enqueueing the root node, since it will always be dequeued first. In line 9 , the next closest object is reported. At that point, some other routine (such as a query engine) can take control, possibly resuming the algorithm at a later time to get the next closest object, or alternately terminating it if no more objects are desired.

Recall that for some types of spatial indexes, a spatial object may span several nodes. In such a case, the algorithm must guard against objects being reported more than once [2]. The test (i.e., the if statement) in line 12 ensures that objects that have already been reported are not put on the queue again. (Note that this 


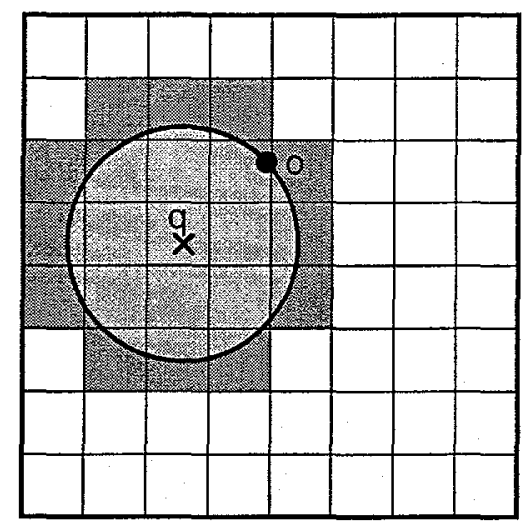

Figure 2: The circle around query object $q$ depicts the search region after reporting $o$ as next nearest object. For simplicity, the leaf nodes are represented by a grid; in most spatial indexes, the shapes of the leaf nodes are more irregular than in a grid. Only the shaded leaf nodes are accessed by the incremental nearest neighbor algorithm. The region with darker shading is where we find the objects in the priority queue.

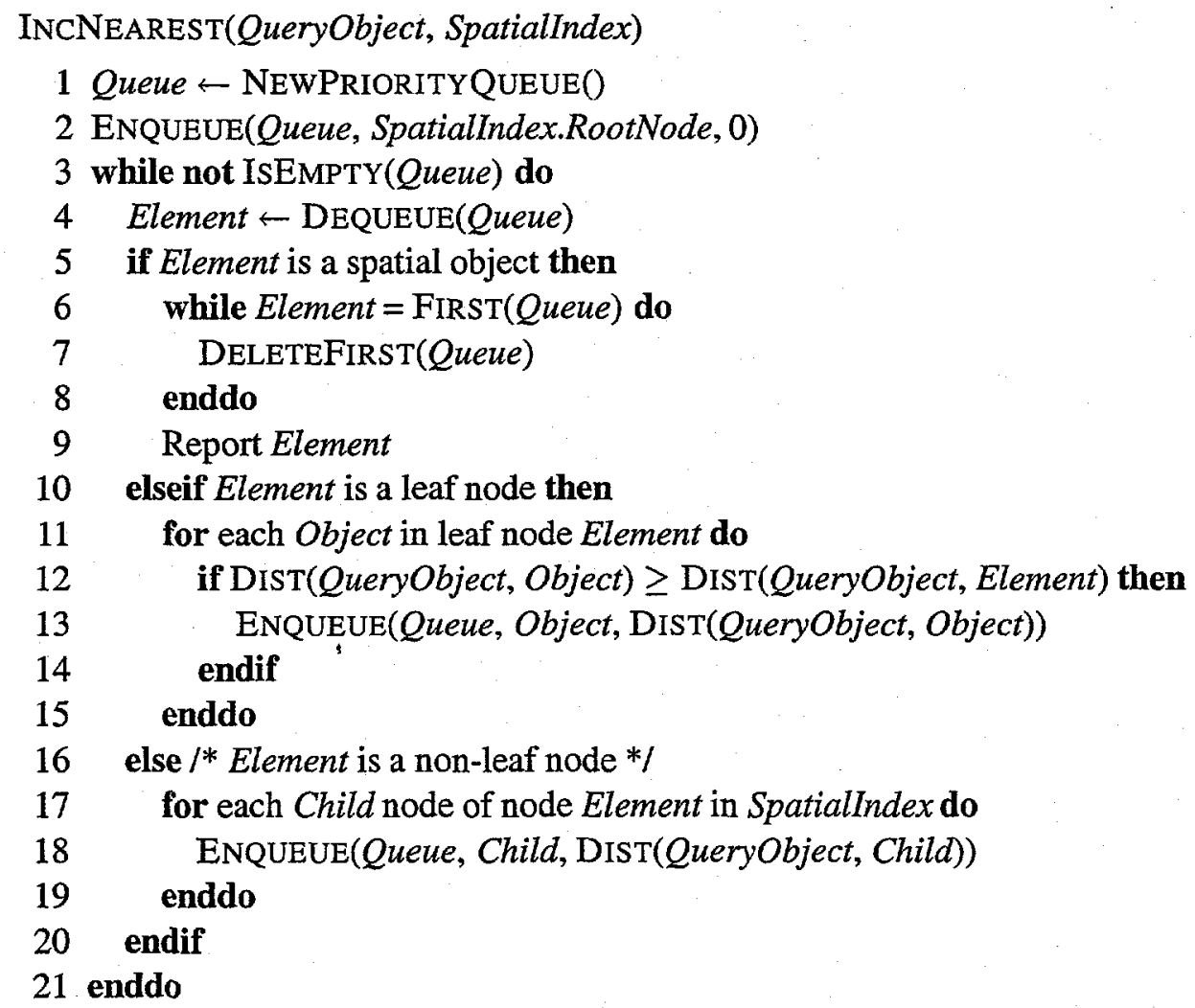

Figure 3: Incremental nearest neighbor algorithm.

test is not needed in the case when Element is a non-leaf node, as it holds implicitly by the assumption that child nodes are fully contained in their parent nodes.) For this to work properly, nodes must be retrieved from the queue before spatial objects at the same distance. Otherwise, an object may be retrieved from the queue 
before a node $n$ containing it that is at the same distance from the query object (this means that the object was contained in another node that has already been dequeued). When the object is then encountered again in node $n$, there is no way of knowing that it has already been reported. The loop in lines 6-8 eliminates duplicate instances of an object from the queue. By inducing an ordering on objects that are at the same distance from the query object, all of the instances of an object will be clustered at the front of the queue when the first instance reaches the front. The reason we check for duplicates in this manner is that for many representations of a priority queue it is not efficient to test for membership. Thus we see that the removal of duplicates is largely a byproduct of the algorithm.

\subsection{Adapting to R-trees}

In this section, we demonstrate how to adapt the general incremental algorithm presented above to R-trees by exploiting some of the unique properties of R-trees. If the spatial objects are stored external to the R-tree, such that leaf nodes contain only bounding rectangles for objects, then this adaptation leads to a considerably more efficient (and conceptually different) incremental algorithm. This enables the bounding rectangles to be used as pruning devices, thereby reducing the disk I/O needed to access the spatial descriptions of the objects. In addition, R-trees store each object just once, making it unnecessary to worry about reporting an object more than once. This also removes the need to enforce the secondary ordering on the priority queue used by the general algorithm (see Section 4.2).

The inputs to the R-tree incremental nearest neighbor algorithm are a query object $q$ and an R-tree $R$ containing a set of spatial data objects. As with the general incremental nearest neighbor algorithm, the data objects as well as the query object may be of any dimension and of arbitrary type (e.g., points, rectangles, polygons, etc.), as long as consistent distance functions are used for calculating the distance from $q$ to data objects and bounding rectangles. In the case of an R-tree, this means that if $e$ is a data object or a rectangle completely contained in rectangle $r$, then $d(q, r) \leq d(q, e)$.

The general algorithm can be used virtually unchanged if object geometry is stored in the R-tree leaf nodes, the only changes being the ones already described. If the spatial objects are stored external to the $\mathrm{R}$ tree, the primary difference from the general algorithm is in the use of the bounding rectangles stored in the leaf nodes. To exploit that information, a third type of queue element is introduced: object bounding rectangle. The distance of an object bounding rectangle is never greater than the distance of the object, provided the distance functions used are consistent. Informally, the modifications to the algorithm are as follows: When an R-tree leaf is being processed in the main loop of the algorithm, instead of computing the real distances of the objects, the distances of their bounding boxes are computed and inserted into the queue. Only when an object's bounding box is retrieved from the queue is the actual distance computed. If the object is closer to the query object than the next element on the priority queue, it can be reported as the next nearest neighbor. Otherwise, the object is inserted into the queue with its real distance.

Figure 4 shows our algorithm. In lines 1-2, the queue is initialized. In line 9, the next closest object is reported. In line 7 , an object $p$ is enqueued with its real distance as the key after it has been determined that there are elements on the queue with a key less than the real distance from $p$ to the query object $q$. If there are no such elements, $p$ is reported as the next nearest object. Line 13 enqueues an object bounding rectangle; brackets around Object signal that it is not the object itself but instead the bounding rectangle along with a pointer to the corresponding object. The general incremental nearest neighbor algorithm had an extra test at this point to guard against reporting duplicates, but that is not needed here.

The R-tree variant given above can be used for any spatial data structure method that separates the storage of bounding rectangles and the actual geometric descriptions of objects. For complex objects, for exam- 
To appear in ACM Transactions on Database Systems (also Univ. of MD Computer Science TR 3919) 10 INCNEAREST(QueryObject, $R$-tree)

1 Queue $\leftarrow$ NEWPRIORITYQUEUE()

2 Enqueue(Queue, R-tree.RootNode, 0 )

3 while not IsEMPTY (Queue) do

4 Element $\leftarrow$ DEQUEUE (Queue)

5 if Element is an object or its bounding rectangle then

6 if Element is the bounding rectangle of Object and not ISEMPTY(Queue) and DIST(QueryObject, Object) $>$ FIRST(Queue).Key then

7 ENQUEUE(Queue, Object, DIST(QueryObject, Object))

8 else

9 Report Element (or if bounding rectangle, the associated object) as the next nearest object

10 endif

11 elseif Element is a leaf node then

12 for each entry (Object, Rect) in leaf node Element do

13 ENQUEUE(Queue, [Object], DIST(QueryObject, Rect))

14 enddo

15 else /* Element is a non-leaf node */

16 for each entry (Node, Rect) in node Element do

17 ENQUEUE(Queue, Node, Dist(QueryObject, Rect))

18 enddo

19 endif

20 enddo

Figure 4: Incremental nearest neighbor algorithm for an R-tree where spatial objects are stored external to the R-tree.

ple polygons, one can even conceive of several levels of refinement, e.g., with the use of orthogonal polygons [17].

\begin{tabular}{|l|r|r|}
\hline Seg. & Dist. & BR Dist. \\
\hline $\mathrm{a}$ & 17 & 13 \\
$\mathrm{~b}$ & 48 & 27 \\
$\mathrm{c}$ & 57 & 53 \\
$\mathrm{~d}$ & 59 & 30 \\
$\mathrm{e}$ & 48 & 45 \\
$\mathrm{f}$ & 86 & 74 \\
$\mathrm{~g}$ & 81 & 74 \\
$\mathrm{~h}$ & 17 & 17 \\
$\mathrm{i}$ & 21 & 0 \\
\hline
\end{tabular}

\begin{tabular}{|l|r|}
\hline BR & Dist. \\
\hline R0 & 0 \\
R1 & 0 \\
R2 & 0 \\
R3 & 13 \\
R4 & 11 \\
R5 & 0 \\
R6 & 44 \\
\hline
\end{tabular}

Table 1: Distances of line segments and bounding rectangles from the query point $q$ in the R-tree of Figure 1 . 


\subsection{Example}

As an example, suppose that we want to find the three nearest neighbors to query point $q$ in the R-tree given in Figure 1, where the spatial objects are line segments which are stored external to the R-tree. Below, we show the steps of the algorithm and the contents of the priority queue. The algorithm must compute the distances between $q$ and the line segments and bounding rectangles. These distances are given in Table 1 ( $B R$ means bounding rectangle). They are based on an arbitrary coordinate system and are approximate. When depicting the contents of the priority queue, the line segments and bounding rectangles are listed with their distances, in increasing order of distance, with ties broken using alphabetical ordering. Bounding rectangles of objects are denoted by the corresponding object names embedded in brackets (e.g., [h]). The algorithm starts by enqueueing R0, after which it executes the following steps:

1. Dequeue $R 0$, enqueue $R 1$ and $R 2$. Queue: $\{(R 1,0),(R 2,0)\}$.

2. Dequeue R1, enqueue $R 3$ and R4. Queue: $\{(R 2,0),(R 4,11),(R 3,13)\}$.

3. Dequeue R2, enqueue R5 and R6. Queue: $\{(R 5,0),(R 4,11),(R 3,13),(R 6,44)\}$.

4. Dequeue R5, enqueue [C] and [i] (i.e., the bounding rectangles of $c$ and $i$ ). Queue: $\{([i], 0)$, $(\mathrm{R} 4,11),(\mathrm{R} 3,13),(\mathrm{R} 6,44),([\mathrm{c}], 53)\}$.

5. Dequeue [ $i$ ]. The distance of $i$ is 21 , which is larger than the distance of $R 4$, so enqueue $i$. Queue: $\{(\mathrm{R} 4,11),(\mathrm{R} 3,13),(\mathrm{i}, 21),(\mathrm{R} 6,44),([\mathrm{c}], 53)\}$.

6. Dequeue $R 4$, and enqueue $[d],[g]$, and $[h]$. Queue: $\{(R 3,13),([h], 17),(i, 21),([d], 30)$, $(R 6,44),([c], 53),([g], 74)\}$.

7. Dequeue R3, enqueue $[a]$ and $[b]$. Queue: $\{([a], 13),([h], 17),(i, 21),([b], 27),([d], 30)$, $(\mathrm{R} 6,44),([\mathrm{c}], 53),([\mathrm{g}], 74)\}$.

8. Dequeue [a]. The distance of $\mathrm{a}$ is 17 , which is not larger than the distance of $[\mathrm{h}]$, so $\mathrm{a}$ is reported as nearest neighbor. Queue: $\{([\mathrm{h}], 17),(\mathrm{i}, 21),([\mathrm{b}], 27),([\mathrm{d}], 30),(\mathrm{R} 6,44),([\mathrm{c}], 53),([\mathrm{g}], 74)\}$.

9. Dequeue [h]. The distance of $h$ is 17 , which is not larger than the distance of $i$, so $h$ is reported as second nearest nieighbor. Queue: $\{(i, 21),([b], 27),([d], 30),(R 6,44),([c], 53),([g], 74)\}$.

10. Dequeue $i$ and report it as third nearest neighbor.

Observe that node R6 is left on the priority queue at the end of the execution. This corresponds to the $k$-nearest neighbor algorithm not being invoked on that node (see Section 5.2). For larger examples, the incremental algorithm will generally achieve more pruning than the $k$-nearest neighbor algorithm, but never less.

Also note that the second and third nearest neighbors were obtained with very little additional work once the nearest neighbor was found. This is often the case with the incremental nearest neighbor algorithm regardless of the underlying spatial index. In other words, once the nearest neighbor has been found, the next few nearest neighbors can be retrieved with virtually no additional work. 


\subsection{Variants}

With relatively minor modifications, the incremental nearest neighbor algorithm can be used to find the farthest object from the query object. In this case, the queue elements are sorted in decreasing order of their distances. This is not enough, though, since objects or nodes contained in a node $n$ are generally at larger distances from the query object $q$ than $n$ is. This means that elements would be enqueued with larger keys than the node they are contained in, which breaks the condition that elements are dequeued in decreasing order of distance. Instead, the key used for a node $n$ on the queue must be an upper bound on the distance from $q$ to an object in the subtree at $n$, e.g., $d_{\max }(q, n)=\max _{p \in n} d_{p}(q, p)$. The function implementing $d_{\max }$ must satisfy a consistency condition similar to that defined above for $d_{n}$; the only difference is that for $d_{\max }$, we replace $\leq$ in the condition by $\geq$.

Another extension to the algorithm is to allow a minimum and a maximum to be imposed on the distances of objects that are reported. However, in order to effectively utilize a minimum, the distance function $d_{\max }$ defined above is needed. Then, a node $n$ is put on the queue only if $d_{\max }(q, n)$ is greater or equal to the minimum desired distance. Notice that in this case, the algorithm performs a spatial selection operation in addition to the ranking.

Figure 5 gives a version of the algorithm with these features added. The arguments Min and Max specify the minimum and maximum desired distance, and DoFarthest is a Boolean variable that is true when the farthest object is desired. In the latter case, negative distances are used as keys for the priority queue, so that elements get sorted in decreasing order of distance. The condition $\operatorname{KeySign}(d-e) \geq 0$ in line 19 of Figure 5 encompasses the conditions $d \geq e$ and $d \leq e$, for when DoFarthest is false and true, respectively. In line 16, the key of the leaf node is assigned to $e$. This is the minimum or maximum distance of the node, depending on the value of DoFarthest. The reason for multiplying the key by KeySign in line 16 is to cancel out the effect of multiplying the value of $d$ by KeySign in line 33, which makes it negative when looking for the farthest objects.

A powerful way of extending the incremental nearest neighbor algorithm is to combine it with other spatial queries and/or restrictions on the objects or nodes. As an example, the algorithm can be combined with a range query by checking each object and node against the range prior to inserting it onto the priority queue, and rejecting those that do not fall in the range. Many such combined queries can be obtained by manipulating the distance functions so that they return special values for objects and nodes that should be rejected.

The incremental nèarest neighbor algorithm can clearly be used to solve the traditional $k$-nearest neighbor problem, i.e., given $k$ and a query object $q$ find the $k$ nearest neighbors of $q$. This is done by simply retrieving $k$ neighbors with the algorithm and terminating once they have all been determined.

\subsection{Analysis}

Performing a comprehensive theoretical analysis of the incremental nearest neighbor algorithm is complicated, especially for high-dimensional spaces. Prior work in this area is limited to the case where both the data objects and the query object are points $[8,26]$. A number of simplifying assumptions were made, e.g., that the data objects are uniformly distributed in the data space. In this section, we discuss some of the issues involved, and sketch a rudimentary analysis for two-dimensional points, based on the one in [26].

We wish to analyze the situation after finding the $k$ nearest neighbors. Let $o$ be the $k^{\text {th }}$ nearest neighbor of the query object $q$, and let $r$ be the distance of $o$ from $q$. The region within distance $r$ from $q$ is called the search region. Since we assume that $q$ is a point, the search region is a circle (or a hypersphere in higher dimensions) 
InCNEAREST(QueryObject, Spatiallndex, Min, Max, DoFarthest)

1 Queue $\leftarrow$ NewPRIORITYQUEUE()

2 ENQUEUE(Queue, SpatialIndex.RootNode, 0)

3 if DoFarthest then

4 KeySign $\leftarrow-1$

5 else

6 KeySign $\leftarrow 1$

7 endif

8 while not ISEMPTY(Queue) do

9 Element $\leftarrow$ DEQUEUE(Queue $)$

10 if Element is a spatial object then

11 while Element $=$ FIRST $($ Queue $)$ do

12 DELETEFIRST(Queue)

13 enddo

14 Report Element

15 elseif Element is a leaf node then

$16 e \leftarrow$ Element.Key*KeySign

17 for each Object in leaf node Element do

$18 \quad d \leftarrow \operatorname{DisT}($ QueryObject, Object)

19 if $d \geq \operatorname{Min}$ and $d \leq \operatorname{Max}$ and KeySign $*(d-e) \geq 0$ then

20 ENQUEUE(Queue, Object, KeySign $* d$ )

21 endif

22 enddo

23 else $/ *$ Element is a non-leaf node *I

24. for each Child node of node Element in Spatiallndex do

$25 \quad d_{\min } \leftarrow \operatorname{MinDisT}($ QueryObject, Child)

$26 \quad d_{\max } \leftarrow$ MAXDIsT(QueryObject,Child)

27 if $d_{\max } \geq$ Min and $d_{\min } \leq$ Max then

28 if DoFarthest then

29

30

31

32

33

34

35

36

37 enddo

$$
d \leftarrow d_{\max }
$$

else

$d \leftarrow d_{\min }$

endif .

ENQUEUE(Queue, Child, KeySign $* d$ )

endif

enddo

endif

Figure 5: Enhanced incremental nearest neighbor algorithm

with radius $r$. Figure 2 depicts this scenario. Observe that all objects inside the search region have already been reported by the algorithm (as the next nearest object), while all nodes intersecting the search region have been examined and their contents put on the priority queue. A further insight can be obtained about the contents of the priority queue by noting that if $n$ is a node that is completely inside the search region, all nodes and objects in the subtree rooted at $n$ have already been taken off the queue. Thus all elements on the 
priority queue are contained in nodes intersecting the boundary of the search region (the dark shaded region in Figure 2).

Before proceeding any further, we point out that the algorithm does not access any nodes or objects that lie entirely outside the search region (i.e., that are farther from $q$ than $o$ is). This follows directly from the queue order and the consistency conditions. In particular, the elements are retrieved from the priority queue in order of distance, and the consistency conditions guarantee that we never insert elements into the queue with smaller distances than that of the element last dequeued. Conversely, any algorithm that uses a spatial index must visit all the nodes that intersect the search region; otherwise, it may miss some objects that are closer to the query object than $o$. Thus we have established that the algorithm visits the minimal number of nodes necessary for finding the $k^{\text {th }}$ nearest neighbor. This can be characterized by saying that the algorithm is optimal with respect to the structure of the spatial index. However, this does not mean that the algorithm is optimal with respect to the nearest neighbor problem; how close the algorithm comes to being optimal in this respect depends on the spatial index.

Generally, two steps are needed to derive performance measures for the incremental nearest neighbor algorithm. First, the expected area of the search region is determined. Then, based on the expected area of the search region and an assumed distribution of the locations and sizes of the leaf nodes, we can derive such measures as the expected number of leaf nodes accessed by the algorithm (i.e., intersected by the search region) or the expected number of objects in the priority queue. Henrich [26] describes one such approach, which uses a number of simplifying assumptions. In particular, it assumes $N$ uniformly distributed data points in the two-dimensional interval $[0,1] \times[0,1]$, the leaf nodes are assumed to form a grid at the lowest level of the spatial index with average occupancy of $c$ points, and the search region is assumed to be completely contained in the data space. Since we assume uniformly distributed points, the expected area of the search region is $k / N$ and the expected area of the leaf node regions is $c / N$. The area of a circle of radius $r$ is $\pi r^{2}$, so for the search region we have $\pi r^{2}=k / N$, which means that its radius is $r=\sqrt{\frac{k}{\pi N}}$. The leaf node regions are squares, so their side length is $s=\sqrt{c / N}$. Henrich [26] points out that the number of leaf node regions intersected by the boundary of the search region is the same as that intersected by the boundary of its circumscribed square. Each of the four sides of the circumscribed square intersects $\lfloor 2 r / s\rfloor \leq 2 r / s$ leaf node regions. Since each two adjacent sides intersect the same leaf node region at a corner of the square, the expected number of leaf node regions intersected by the search region is bounded by

$$
4(2 r / s-1)=4\left(\frac{2 \sqrt{k /(\pi N)}}{\sqrt{c / N}}-1\right)=4\left(2 \sqrt{\frac{k}{\pi c}}-1\right) .
$$

It is reasonable to assume that, on the average, half of the $c$ points in these leaf nodes are inside the search region, while half are outside. Thus the expected number of points remaining in the priority queue (the points in the dark shaded region in Figure 2) is at most

$$
\frac{c}{2} 4\left(2 \sqrt{\frac{k}{\pi c}}-1\right)=2 c\left(2 \sqrt{\frac{k}{\pi c}}-1\right)=\frac{4}{\sqrt{\pi}} \sqrt{c k}-2 c \approx 2.26 \sqrt{c k}-2 c
$$

The number of points inside the search region (the light shaded region in Figure 2) is $k$. Thus the expected number of points in leaf nodes intersected by the search region is at most $k+2.26 \sqrt{c k}-2 c$. Since each leaf node contains $c$ points, the expected number of leaf nodes that were accessed to get these points is bounded by $k / c+2.26 \sqrt{k / c}-2$.

To summarize, the expected number of leaf node accesses is $O(k+\sqrt{k})$ and the expected number of objects in the priority queue is $O(\sqrt{k})$. Intuitively, the "extra work" done by the algorithm comes from the 
boundary of the search region. Roughly speaking, the $k$ term in the expected number of leaf node accesses accounts for the leaf nodes completely inside the search region, while the $\sqrt{k}$ term accounts for the leaf nodes intersected by the boundary of the search region. The points on the priority queue lie outside the search region (since otherwise they would have been taken off the queue) but inside leaf nodes intersected by the boundary of the search region. If the average leaf node occupancy and average node fan-out are fairly high (say 50 or more), the number of leaf node accesses dominates the number of non-leaf node accesses, and the number of objects on the priority queue greatly exceeds the number of nodes on the queue. Thus we can approximate the total number of node accesses and total number of priority queue elements by the number of leaf node accesses and the number of objects on the priority queue. However, the traversal from the root of the spatial index to a leaf node containing the query object will add an $O(\log N)$ term to both of these measures.

If the spatial index is disk-based, the cost of disk accesses is likely to dominate the cost of priority queue operations. However, if the spatial index is memory-based, the priority queue operations are the single largest cost factor for the algorithm. In typical priority queue implementations (e.g., binary heap), the cost of each insertion and deletion operation is $O(\log m)$ where $m$ is the size of the priority queue. The number of objects inserted into the priority queue is $O(k+\sqrt{k})$, each for a cost of $O(\log \sqrt{k})$ (since the expected size is bounded by $O(\sqrt{k})$ ), for a total cost of $O(k+\sqrt{k}) \cdot O(\log \sqrt{k})=O(k \log k)$ (again, if we take the non-leaf nodes into account, this becomes somewhat more complicated).

The analysis that we have outlined is based on assumptions that generally do not hold in practice. In particular, the data is rarely uniformly distributed and the search region often extends beyond the data space. Nevertheless, our analysis allows fairly close predictions of actual behavior for two-dimensional point data even when these assumptions do not hold. For higher dimensions the situation is somewhat more complicated. A detailed analysis in that context is presented in [8].

\subsection{Correctness}

Now let us turn to the correctness of the algorithm in Figure 3. We ignore for the moment the issue of reporting an object more than once. Given a data object $o$, define its ancestor set, denoted by $A(o)$, to include $o$ itself, leaf nodes $n$ that contain $o$ for which $d_{o}(q, o) \geq d_{n}(q, n)$ (at least one such node is guaranteed to exist by the consistency of the distance functions), and all ancestors $n^{\prime}$ of $n$. Applied recursively, the consistency property ensures that $d_{o}(q, o) \geq d_{n}\left(q, n^{\prime}\right)$. The elements in $A(o)$ can be interpreted as representing the object $o$. The following theorem guarantees that an unreported object always has a representative on the queue. This directly implies that every object will eventually be reported, since only bounded numbers of objects and nodes are ever put on the queue.

Theorem Let $R$ be the set of objects already reported, and $Q$ the set of elements on the queue. The following is an invariant for the outer while-loop of INCNEAREST: For each object $o$ in Spatiallindex, we have $A(o) \cap(Q \cup R) \neq \emptyset$ (i.e., at least one element in $A(o)$ is in $Q$ or in $R$ ).

Proof: We prove the theorem for an arbitrary object $o$ by induction. Since we choose $o$ arbitrarily, the proof holds for all objects. The induction is on the number of loop executions. If we can show that the invariant holds before the first execution, and that no loop execution falsifies it (i.e., makes it not hold after the execution of the loop, assuming that it held before the execution), then we have shown that the invariant always holds. Clearly, it holds initially, as the only element on the queue is the root node of SpatialIndex, and the root is an ancestor of all nodes and thus is in $A(o)$ for $o$. 
Now assume that the invariant holds at the beginning of an execution of the while-loop. We will show that it also holds at the end of it. If $o \in R$ (i.e., $o$ has been reported), the invariant trivially holds, as $o$ will not be affected during the loop execution. Otherwise, by the assumption that the invariant holds, there exists some $a \in A(o)$ such that $a \in Q$. The invariant is unaffected if the next element to be dequeued is not $a$, so let us assume that $a$ will be dequeued next.

If $a=o$, then $o$ is subsequently reported, thereby moving from $Q$ to $R$, and the invariant is maintained. If $a$ is a node, we consider the case of a leaf and non-leaf node separately:

1. If $a$ is a leaf node, the for-loop at line 11 enqueues all objects with a distance from $q$ of at least $d_{n}(q, a)$ (i.e., at least DIST (Query Object, Element)). Since $o$ is stored in $a$ (recall that $a \in A(o))$ and since $d_{o}(q, o) \geq d_{n}(q, a)$ by the construction of $A(o), o$ is indeed put on the queue.

2. If $a$ is a non-leaf node, then all its child nodes are enqueued. Since $a$ is in $A(o)$, at least one of the child nodes of $a$ is in $A(o)$ (i.e., $a$ is an ancestor of a leaf node $n$ that contains $o$ ), maintaining the invariant.

Thus we see that for both leaf and non-leaf nodes, at least one of the enqueued elements is in $A(o)$. Thus the invariant is maintained for object $o$. Since $o$ was chosen arbitrarily, we have thus shown that the invariant holds for all objects.

As mentioned, the theorem guarantees that an unreported object always has a representative on the queue. Since elements are retrieved from the queue in order of distance, and all elements in $A(o)$ are no farther from the query point than $o$, at some point $o$ will be put on the queue and eventually reported. Also, when $o$ is reported, it is indeed the next closest object to $q$. If not, then there exists an unreported object $o^{\prime}$ closer to $q$. However, since all representatives of $o^{\prime}$ are also closer to $q$ than $o$ is, at least one of them would be dequeued before $o$, contradicting the assumption that $o$ was most recently dequeued.

The correctness of the duplicate removal (lines 6-8 in Figure 3) follows directly from the ordering imposed on the priority queue. Thus the only way an object can be reported more than once is if it is inserted again into the queue after it has been reported. However, this is avoided by the test in line 12, and the fact that nodes are always processed before objects at the same distance from the query object.

\subsection{Priority Queue}

The cost of priority queue operations plays a role in the performance of the incremental nearest neighbor algorithm. The larger the queue size gets, the more costly each operation becomes. Also, if the queue gets too large to fit in memory, its contents must be stored in a disk-based structure instead of in memory, making each operation even more costly. An example of the worst case of the queue size for the R-tree incremental nearest neighbor algorithm arises when all leaf nodes are within distance $d$ from the query object $q$, while all data objects are farther away from $q$ than $d$. This is shown in Figure 6 where the query object as well as the data objects are points. In this case, all leaf nodes must be processed by the incremental algorithm, and all data objects must be inserted into the priority queue before the nearest neighbor can be determined. Note that any nearest neighbor algorithm that uses this R-tree has to visit all the leaf nodes, since the nearest neighbor is farther away from the query object than all the leaf nodes, and there is no other way to make sure that we have seen the nearest neighbor. Furthermore, note that a worst case like that depicted in Figure 6 is highly unlikely to arise in practice since it depends on a particular configuration of both the data objects and the query object. 


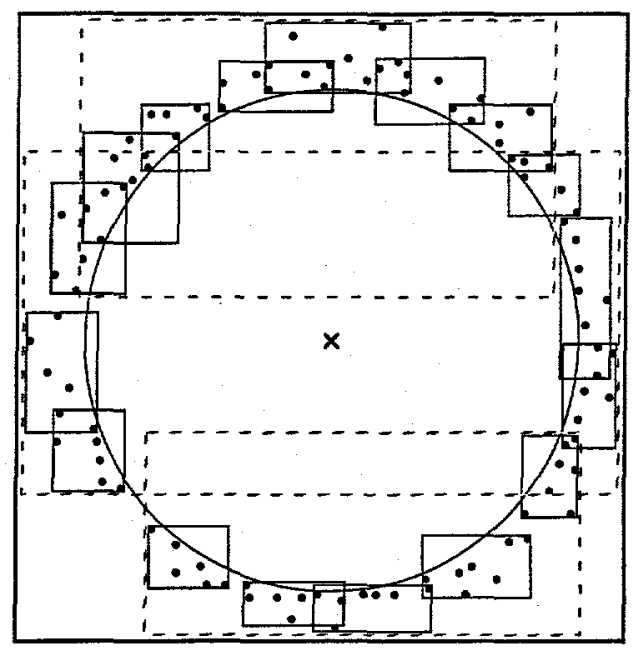

Figure 6: An example of an R-tree of points with node capacity of 8, showing a worst case for nearest neighbor search.

As pointed out in Section 4.6, the objects on the priority queue are contained in leaf nodes intersected by the boundary of the search region. For two-dimensional uniformly distributed data points we mentioned that the expected number of points in the priority queue when finding the $k$ nearest neighbors is $O(\sqrt{k})$. Even if $k$ is as large as several hundred million (of course, the data set has to be even larger than $k$ ), the size of the priority queue is still manageable for keeping in memory. However, more complex objects than points and very skewed data distributions may cause larger proportions of the objects to be inserted into the priority queue. Moreover, as the number of dimensions grows, the size of the priority queue as a function of $k$ tends to get larger (see Section 7). Thus we must be prepared to deal with a very large priority queue.

In cases where the priority queue exceeds the size of available memory it must be stored in whole or in part in a disk-resident structure. One possibility is to use a B-tree structure to store the entire contents of the priority queue. With proper buffer management, we should be able to arrange that the B-tree nodes that store elements with smaller distances (which will get dequeued early) will be kept in memory. However, we believe that when the priority queue actually fits in memory, using B-trees will be considerably slower than using fast heap-based approaches [20], since the B-tree must expend more work on maintaining the queue elements in fully sorted order. In contrast, heap methods impose a much looser structure on the elements. A hybrid scheme for storing the priority queue, where a portion of the priority queue is kept in memory and a portion is kept on disk, therefore seems more appropriate.

A simple way to implement a hybrid memory/disk-based priority queue is to partition the queue elements based on distance. Below, we outline how this can be done. The contents of the priority queue are split into three tiers. The first tier is kept in a memory-based heap structure, while the second and third tiers are kept in a disk file (the difference is that a little more structure is imposed on the contents of the second tier). Let $D_{0}, D_{1}, D_{2}, \ldots, D_{m}$ be some monotonically increasing sequence, where $D_{0}=0$ and $D_{m}$ is an upper bound on the largest possible distance from the query object $q$ to a data object (e.g., the distance from $q$ to the farthest corner of the data space). We use the sequence to define ranges of distances, and associate different ranges with the various tiers. When a new element with a distance of $r$ from the query object is inserted into the priority queue, that element gets added to the tier whose associated distance range matches $r$. Initially, tier 1 is associated with the distance range $\left[D_{0}, D_{1}\right)$ (i.e., queue elements in this range are stored in the memorybased heap structure), tier 2 with the range $\left[D_{1}, D_{p}\right)$, and tier 3 with the range $\left[D_{p+1}, D_{m}\right)$. The contents of tier 2 are divided into $p$ ranges, $\left[D_{1}, D_{2}\right),\left[D_{2}, D_{3}\right), \ldots,\left[D_{p}, D_{p+1}\right)$. The value of $p$ depends on how many 
ranges it is cost-effective to maintain, but it can be as high as $m$. When tier 1 is exhausted, we move the elements in distance range $\left[D_{1}, D_{2}\right)$ from tier 2 to tier 1 and associate tier 1 with that distance range. The next time tier 1 is exhausted, we move elements in distance range $\left[D_{2}, D_{3}\right)$ into tier 1 , and so on. If this happens often enough, eventually we will exhaust tier 2 . When this happens, we scan the entire contents of tier 3 and rebuild tiers 1 and 2 with new ranges. Note that moving elements from tier 3 to tier 2 only when tier 2 is exhausted rather than each time tier 1 is exhausted reduces the number of scans of tier 3 , which may contain a large number of elements.

In general, when the distance of the elements at the head of the priority queue is in the range $\left[D_{i}, D_{i+1}\right)$ for some $i=0, \ldots, m$ (i.e., all neighbors with distances less than $D_{i}$ from $q$ have already been reported), then tier 1 is associated with the range $\left[D_{i}, D_{i+1}\right)$, tier 2 with the range $\left[D_{i+1}, D_{i+s+1}\right)$, and tier 3 with the range $\left[D_{i+s+2}, D_{m}\right)$, where $s=p-(i \bmod p)$. We keep the elements in tier 2 in a set of linked lists, one for each interval $\left[D_{j}, D_{j+1}\right)$ where $j=i+1, \ldots, i+s$. In order to save on disk I/Os, we can associate a buffer with each of these linked lists and group elements into pages of fixed size. An alternative to using linked lists within the same file is to use a separate file for each range. Also, rather than associating range $\left[D_{i+1}, D_{i+s+1}\right)$ with tier 2 , we can associate with it the entire range $\left[D_{i+1}, D_{i+p+1}\right)$, so that newly inserted elements in that range get inserted into tier 2 rather than tier 3. However, we still do not want to scan tier 3 each time we exhaust tier 1 , so tier 3 will also contain elements in the range $\left[D_{i+s+1}, D_{i+p+1}\right)$. These elements get moved into tier 2 when tier 3 gets scanned next, which happens when $i \bmod p=0$.

A variation of this technique is to use an additional tier, between tier 1 and tier 2 , in which elements are stored in an unsorted list in memory. The idea is that because we limit the size of the memory-based heap, the insertion and deletion operations on it are less expensive. Keeping the new tier 2 in memory but outside the heap makes it inexpensive to add elements to it (i.e., this does not require disk L/Os), but if only a small number of neighbors is requested, they will never need to be placed on the heap.

The remaining question is how to choose the sequence $D_{0}, D_{1}, D_{2}, \ldots, D_{m}$. A naive way is to simply guess some distance threshold $D_{T}$, and then set $D_{i}=i \cdot D_{T}$. Alternatively, we can assume some data distribution and use it to derive an appropriate sequence. For example, recall from Section 4.6 that under the assumptions made there, the expected number of leaf nodes intersected by the boundary of a search region of radius $r$ is bounded by $4(2 r / s-1)$, where $s=\sqrt{c / N}$ is the expected side length of each leaf node region. Again, assuming that half of the points in these nodes (i.e., $c / 2$ ) are outside the search region, the expected number of points on the priority queue is at most $\frac{c}{2} 4(2 r / s-1)=2 c(2 r / s-1)$. Assuming that we have space in memory for $M$ priority queue elements means that $D_{i}$ must satisfy the equation $i \cdot M=2 c\left(2 D_{i} / s-1\right)$, so that

$$
D_{i}=\left(\frac{i \cdot M}{2 c}+1\right) / 2 \cdot s
$$

Of course, this derivation is based on assumptions that generally do not hold in practice. Nevertheless, it should work fairly well in practice for two-dimensional points. Moreover, it gives an indication of how to obtain such a sequence for other ways of analyzing the size of the priority queue.

\section{$5 \quad k$-Nearest Neighbor Search in R-trees}

An alternative approach to nearest neighbor search in R-trees was proposed in [45]. This approach is applicable when finding the $k$ nearest neighbors where $k$ is fixed in advance. This is in contrast to the incremental nearest neighbor algorithm, where $k$ does not have to be fixed in advance. The key idea of the $k$-nearest neighbor algorithm is to maintain a global list of the candidate $k$ nearest neighbors as the R-tree is traversed in a depth-first manner. As we will see, the fact that the $k$-nearest neighbor algorithm employs a pure depth- 
first traversal means that at any step the algorithm can only make local decisions about which node to visit (i.e., the next node to visit must be a child node of the current node), whereas our incremental nearest neighbor algorithm makes global decisions based on the contents of the priority queue (i.e., it can choose among the child nodes of all nodes that have already been visited).

In this section, we first describe a somewhat simplified version of the $k$-nearest neighbor algorithm in [45] and show an example of its execution. Next, we prove that our simplified version is in fact equivalent to the algorithm presented in [45]: Both versions visit the same nodes in the R-tree. Finally, we show how the $k$-nearest neighbor algorithm can be transformed in a sequence of steps into an incremental algorithm.

\subsection{Algorithm Description}

In the $k$-nearest neighbor algorithm [45], the $\mathrm{R}$-tree is traversed in a depth-first manner. The complications mentioned in Section 4 that arise in performing nearest neighbor search with a depth-first traversal are overcome by maintaining a list of the candidate $k$ nearest neighbors. In particular, once we reach a leaf node containing the query object, we insert the contents of that node into the candidate list, and unwind the recursive traversal of the tree. Once the candidate list contains $k$ members, the largest distance of any of its members from the query object can be used to prune the search.

Figure 7 shows the $k$-nearest neighbor algorithm. In the figure, NearestList denotes the list of the $k$ candidate nearest neighbors, and NearestList.MaxDist denotes the largest distance from the query object of any of the members of NearestList; if NearestList contains fewer than $k$ members, this distance is taken to be $\infty$. When an object is inserted into NearestList in line 4 of KNEARESTTRAVERSAL, an existing member is replaced if the list already contains $k$ members. In particular, we replace the member that is farthest from the query object (i.e., the one at distance NearestList.MaxDist). Before inserting an object into NearestList, we first make sure that its distance from the query object is smaller than NearestList.MaxDist (line 3 of KNEARESTTRAVERSAL). Note that NearestList.MaxDist decreases monotonically as more objects are inserted into the list, since we always replace objects with objects closer to the query object.

In KNEARESTTRAVERSAL, if Node is a non-leaf node, its child nodes are visited in order of distance from the query object. This is done by building the list ActiveBranchList of the entries in Node, and sorting it by distance from the query object (see Section 5.3 for different ways of defining this order). Next, we iterate through the list (in the sorted order) and recursively invoke KNEARESTTRAVERSAL on the child nodes. Once the distance of Child from the query object is larger than NearestList.MaxDist, we ignore Child and the rest of the entries in ActiveBranchList. We can do this because this means that no object in the subtree of Child (or the remaining entries in ActiveBranchList) will get inserted into NearestList.

The difference between the $k$-nearest neighbor algorithm in Figure 7 and the original presentation in [45] is in the treatment of ActiveBranchList. We use only one pruning strategy to eliminate entries from consideration, by comparing their distances to NearestList.MaxDist, while [45] identifies two other pruning strategies. However, in Section 5.4 we will show that the other two pruning strategies in fact do not allow any more pruning than the one that we use.

If the objects are stored outside the R-tree (i.e., the R-tree leaf nodes contain bounding rectangles and object references), a minor optimization can be made in line 4 of KNEARESTTRAVERSAL. We first compute the distance from the query object to the bounding rectangle. Only if this distance is less than NearestList.MaxDist do we compute the real distance from Object to the query object. Otherwise, Object is not accessed, thereby potentially saving a disk $\mathrm{L} / \mathrm{O}$, as in this scenario the objects are stored outside the R-tree. Recall that $d(q, r) \leq d(q, o)$ if $r$ is a bounding rectangle of the object $o$, i.e., the distance of $o$ from $q$ is never 
To appear in ACM Transactions on Database Systems (also Univ. of MD Computer Science TR 3919) 20 KNEAREST $(k$, QueryObject, SpatialIndex)

1 NearestList $\leftarrow \operatorname{NEWLIST}(k)$

2 KNEARESTTRAVERSAL(NearestList, $k$, Queryobject, SpatialIndex.RootNode)

3 return NearestList

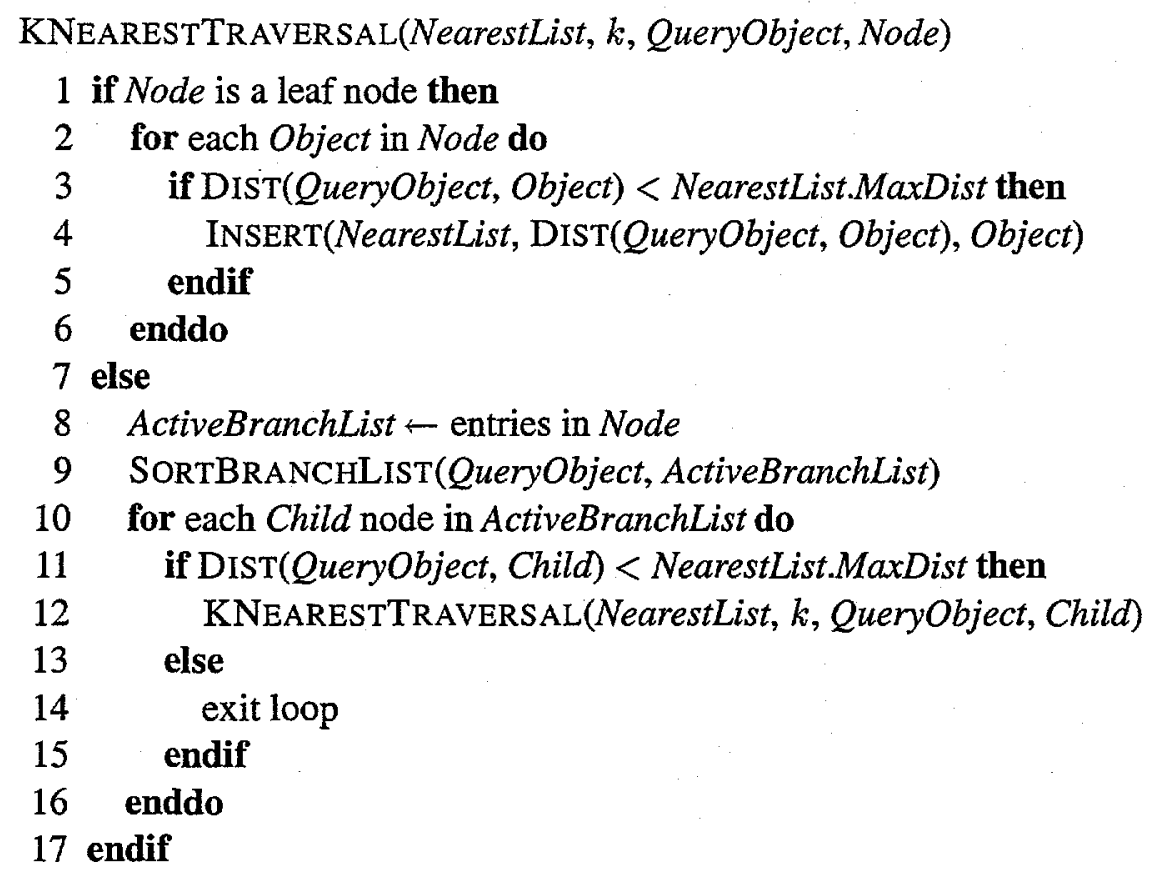

Figure 7: $k$-nearest neighbor algorithm.

less than the distance of $r$ from $q$.

In [45] it is suggested that a sorted buffer be used to store NearestList. However, we found that for large values of $k$, the manipulation of NearestList started to become a major factor in the execution time of the algorithm. Therefore, we replaced the sorted buffer with a simple priority queue structure, sorted in decreasing order of distance, thereby making it easy to replace the farthest object.

\subsection{Example}

As an example of the algorithm, we describe its use in finding the three nearest neighbors to query point $\mathrm{q}$ in the R-tree given in Figure 1. Below, we show the steps of the algorithm and the contents of the ActiveBranchLists and of NearestList. The example makes use of the distances between $q$ and the line segments and bounding rectangles given in Table 1. An invocation with node $x$ is denoted by $k-\mathrm{NN}(x)$. We start by applying it to the root of the R-tree, RO. Next, we describe the subsequent invocations of the algorithm. Each of the line segment elements in NearestList is listed along with its distance from q. In our specification of NearestList we also list the maximum distance used for pruning (i.e., NearestList.MaxDist). Initially, NearestList is empty and the maximum distance is $\infty$.

1. $k-\mathrm{NN}(\mathrm{RO})$ : ActiveBranchList for $\mathrm{RO}$ is (R1, R2).

(a) $k$-NN(RI): ActiveBranchList for RI is (R4, R3). 
i. $k$-NN(R4): insert $\mathrm{d}, \mathrm{g}, \mathrm{h}$ on NearestList: $\{(\mathrm{h}, 17),(\mathrm{d}, 59),(\mathrm{g}, 81): 81\}$.

ii. $k$-NN(R3): insert $\mathrm{a}, \mathrm{b}$ in NearestList (replacing $\mathrm{d}, \mathrm{g}):\{(\mathrm{h}, 17),(\mathrm{a}, 17),(\mathrm{b}, 48): 48\}$.

(b) $k$-NN(R2): ActiveBranchList for R2 is (R5, R6).

i. $k$-NN(R5): i replaces $b$, but $c$ is too distant: $\{(h, 17),(a, 17),(i, 21): 21\}$.

ii. $k$-NN(R6): this invocation does not occur, as the distance of $\mathrm{R} 6$ from $\mathrm{q}$ is $\geq 21$.

The final contents of NearestList is $\{(h, 17),(a, 17),(i, 21)\}$ which is returned as the list of the three nearest neighbors of $q$.

\subsection{Node Ordering and Metrics}

The ordering used to sort the elements in ActiveBranchList in Figure 7 can be based on various metrics for measuring the distances between QueryObject and the elements' bounding rectangles. Two such metrics are considered in [45], MINDIST and MINMAXDIST. For bounding rectangle $r$ of node $n$, MINDIST $(q, r)$ is the minimum possible distance from $q$ to an object in the subtree rooted at $n$, while $\operatorname{MiNMAXDIST}(q, r)$ is the maximum distance from $q$ at which an object in the subtree rooted at $n$ is guaranteed to be found (i.e., it is the minimum of the maximum distances at which an object can be found). MINDIST and MiNMAXDIST are calculated by using the geometry (i.e., position and size) of the bounding rectangle $r$ of node $n$ and do not require examining the actual contents of $n$. A more precise definition is given as follows. $\operatorname{MinDisT}(q, r)$ is the distance from $q$ to the closest point on the boundary of $r$ (not necessarily a corner), while MinMaXDist $(q, r)$ is the distance from $q$ to the closest corner of $r$ that is "adjacent" to the corner farthest from $q$. Figure 8 shows two examples of the calculation of MINDIST and MINMAXDIST, which are shown with a solid and a broken line, respectively. Notice that for the bounding rectangle in Figure 8a the distance from $q$ to $a$ is less than the distance from $q$ to $b$, thereby accounting for the value of MINMAXDIST being equal to the former rather than the latter, while the opposite is true for Figure $8 \mathrm{~b}$. In some sense, the two orderings represent the optimistic (MINDIST) and the pessimistic (MINMAXDIST) choice. To see this, observe that if $r_{1}$ and $r_{2}$ are minimum bounding rectangles in order of increasing value of MINDIST (i.e., $\left.\operatorname{MinDiST}\left(q, r_{1}\right) \leq \operatorname{MinDIST}\left(q, r_{2}\right)\right)$, then at best, $r_{1}$ contains an object $o_{1}$ at a distance close to its MINDIST value, such that $\operatorname{DIST}\left(q, o_{1}\right) \leq \operatorname{MinDiST}\left(q, r_{2}\right)$; but this need not hold, as $r_{2}$ may contain an object closer to $q$. If $r_{1}$ and $r_{2}$ are in order of increasing MINMAXDIsT value, on the other hand, then in the worst case, the object in $r_{1}$ nearest to $q$ is at distance $\operatorname{MinMAXDist}\left(q, r_{1}\right)$, which is no larger than $\operatorname{MinMaxDIST}\left(q, r_{2}\right)$.

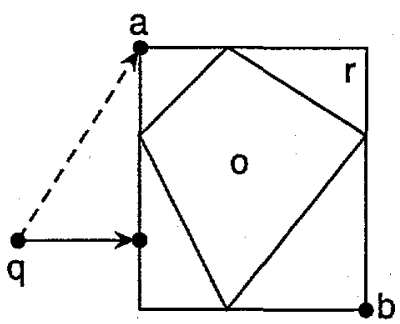

(a)

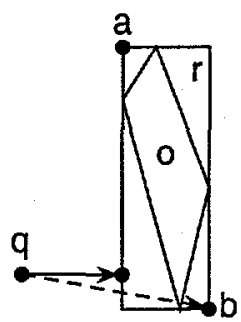

(b)

Figure 8: An example of MiNDIST (solid line) and MINMAXDIST (broken line) for a bounding rectangle $r$. The distance of the object $o$ from $q$ is bounded from below by $\operatorname{MinDist}(q, r)$ and from above by $\operatorname{MinMAXDist}(q, r)$. Notice that in (b) point $b$ is closer to $q$ than point $a$ while this is not the case in (a). 
Experiments reported in [45] showed that ordering ActiveBranchList using MINDIST consistently performed better than using MINMAXDIST. This was confirmed in our experiments, although we do not include that result in Section 6 which describes our experimental findings. We suspect that this indicates that the optimism inherent in MINDIST usually provides a better estimate of the distance of the nearest object than the pessimism inherent in MINMAXDIST, so that MINDIST order will in general lead to the nearest object(s) being found earlier in the ActiveBranchList. In this paper we therefore assume that ActiveBranchList is ordered using MiNDIST. In fact, the algorithm in Figure 7 depends on this, as we discuss at the end of Section 5.4.

The metrics have other uses, regardless of which one is used for ordering ActiveBranchList. Since MrNDIST represents the minimum distance at which an object could be found in a bounding rectangle $r$, it provides a means of pruning nodes from the search, given that a bound on the maximum distance is available. On the other hand, for any bounding rectangle $r, \operatorname{MinMAXDIST}(q, r)$ is an upper bound on the distance of the object $o$ nearest to $q$. It should be clear that MINMAXDIST by itself does not help in pruning the search, as objects closer to $q$ could be found in elements of $n$ at positions with higher MINMAXDIST values. Moreover, since it only bounds the distance at which the closest element can be found, this property is of limited value, as it is only useful when we are seeking the nearest neighbor (i.e., $k=1$ ).

\subsection{Pruning Strategies}

As already mentioned, the algorithm of [45] employs a set of three pruning strategies to prune entries from ActiveBranchList as the entries are processed. Two classes of pruning strategies are identified in [45], termed downward pruning and upward pruning. In downward pruning, entries on ActiveBranchList are eliminated prior to processing the nodes (i.e., before entering the for-loop in line 10 of KNEARESTTRAVERSAL in Figure 7). In upward pruning, entries on ActiveBranchList are eliminated after processing each node (i.e., after returning from the recursive call to KNEARESTTRAVERSAL in line 10 in Figure 7). Of the three pruning strategies discussed in [45], two are said to be applicable to downward pruning and one to upward pruning. Below, we will discuss these three pruning strategies in turn, and show that one of them is sufficient when used in a combination of upward and downward pruning ${ }^{3}$.

Strategy 1 is used in downward pruning. It allows pruning an entry from ActiveBranchList whose bounding rectangle $r_{1}$ is such that $\operatorname{MinDisT}\left(q, r_{1}\right)>\operatorname{MinMAXDIST}\left(q, r_{2}\right)$, where $r_{2}$ is some other bounding rectangle in ActiveBranchList. However, as already pointed out, using MINMAXDIST for pruning is of limited value as it is only useful when $k=1$.

Strategy 2 prunes an object $o$ when $\operatorname{DisT}(q, o)>\operatorname{MinMAXDIST}(q, r)$, where $r$ is some bounding rectangle. Again, this strategy is only applicable to $k=1$. This strategy is claimed to be of use in downward pruning in [45], but its inclusion is somewhat puzzling, since it does not help in pruning nodes from the search. It is possible that the authors intended strategy 2 to be used to prune objects in leaf nodes. However, this does not appear to be particularly fruitful, since it still requires the objects to be accessed and their distances from $q$ calculated. Another possible explanation for the inclusion of this strategy is that it can be used to discard the nearest object found in a subtree $s$ in ActiveBranchList after $s$ has been processed. However, the purpose of this is not clear, since a better candidate will replace this object later on, anyway.

Strategy 3 prunes any node from ActiveBranchList whose bounding rectangle $r$ is such that MINDIST $(q, r)>$ NearestList.MaxDist. It is applicable regardless of the value of $k$ and in both downward and upward pruning. Note that although strategy 3 is not explicitly labeled as a downward pruning strategy in [45], its use in

\footnotetext{
${ }^{3}$ It may appear that we use this pruning strategy only for upward pruning in line 11 of KNEARESTTRAVERSAL in Figure 7. However, since the condition is checked before the recursive call to KNEARESTTRAVERSAL, the if statement actually does both upward and downward pruning.
} 
downward pruning is noted. In particular, before entering the for-loop in line 10 of KNEARESTTRAVERSAL in Figure 7, we can eliminate entries in ActiveBranchList with distances larger than NearestList.MaxDist (no pruning will occur, though, unless NearestList contains at least $k$ entries).

Recalling that strategy 1 is only applicable when $k=1$, it can be shown that even in this case applying strategy 3 in upward pruning eliminates at least as many bounding rectangles as applying strategy 1 in downward pruning. To see this, let $r$ be the bounding rectangle in ActiveBranchList with the smallest MiNMAXDIST value. Using strategy 1, we can prune any entry in ActiveBranchList with bounding rectangle $r^{\prime}$ such that $\operatorname{MinDisT}\left(q, r^{\prime}\right)>\operatorname{MinMAXDisT}(q, r)$. However, strategy 1 will not prune $r$ or any entry in $A c$ tiveBranchList preceding it, regardless of the ordering used. If ActiveBranchList is ordered based on MiNMAXDIST, this clearly holds, since $\operatorname{MinDisT}(q, r) \leq \operatorname{MinMaXDIST}(q, r)$. If ActiveBranchList is ordered based on MINDIST, the nodes preceding $r$ have MINDIST values smaller than that of $r$, so their MiNDIST values must also be smaller than $\operatorname{MinMaxDist}(q, r)$. Now, let us see what entries can be pruned from ActiveBranchList by strategy 3 after processing the node corresponding to $r$. In particular, at that point, $\operatorname{DisT}(q, o) \leq \operatorname{MinMAXDIST}(q, r)$ where $o$ is the candidate nearest object; this follows directly from the definition of MinMaXDisT. Therefore, when strategy 3 (based on $\operatorname{DIST}(q, o)$ ) is now applied to ActiveBranchList, it will prune at least as many entries as strategy 1 (based on $\operatorname{MinMAXDIST}(q, r)$ ).

The fact that we have eliminated strategies 1 and 2 , and we are interested in finding more than $k$ neighbors, implies that MINMAXDIST is not necessary for pruning as it is not involved in strategy 3. Thus, assuming that MINMAXDIST is not used for node ordering, the CPU cost of the algorithm is reduced, since we do not have to compute the MINMAXDIST value of each bounding rectangle; this is especially important because MINMAXDIST is more expensive to compute than MINDIST. We also observe that there is really no need to distinguish between downward and upward pruning in the sense that there is no need to explicitly remove items from ActiveBranchList. Instead, we just test each element on ActiveBranchList when its turn comes. If ActiveBranchList is ordered according to MINDIST, then once we prune one element, we can terminate all computation at this level, as all remaining elements have larger MINDIST values. This is exactly what we do in the if statement in line 11 of KNEARESTTRAVERSAL in Figure 7.

\subsection{Transformation}

In this section we show how the $k$-nearest neighbor algorithm can be transformed into an incremental algorithm, and that the result is identical to our R-tree incremental algorithm. This discussion reveals the main difference between the two algorithms, namely that the control structure of the $k$-nearest neighbor algorithm is fragmented among the nodes on the path from the root to the current node (as specified in the ActiveBranchList of each invocation of the algorithm), while the incremental nearest neighbor algorithm employs a unified control structure embodied in its priority queue.

Recall that the R-tree $k$-nearest neighbor algorithm traverses the R-tree in a depth-first manner. It keeps track of the state of the traversal (i.e., which nodes or bounding rectangles it has yet to process) by use of an ActiveBranchList for each level (note that at most one node is active at each level at any given time). In addition, in its original formulation (i.e., assuming a sorted buffer implementation) it keeps track of the distances from the query object of the data objects that it has seen by use of NearestList sorted in increasing order of distance from the query object. Output of the $k$ nearest neighbors only occurs at the end of the traversal since the R-tree is being traversed in its entirety (subject to the pruning of nodes in ActiveBranchList).

If we want to transform the R-tree $k$-nearest neighbor algorithm into an incremental algorithm, we need to also keep track of the nodes in the R-tree that have been seen (i.e., inserted into an ActiveBranchList) but not processed. These are the elements of the various instances of ActiveBranchList; let $B$ denote their union. 
We assume that elements are removed from NearestList as they are processed. With the aid of $B$, it is now possible to tell if the first element $o$ in NearestList should be reported as the next nearest neighbor to $q$. In particular, this is the case if $o$ is closer to $q$ than the closest node in $B$, as all objects not yet encountered are in subtrees of nodes in $B$. Without the global knowledge that $B$ embodies, it is not possible to report even the nearest neighbor until we have unwound the recursive traversal of the algorithm up to the root node of the R-tree, because before then we do not know what is in the other subtrees of the root.

The $k$-nearest neighbor algorithm can be modified to maintain this global unprocessed node list $B$, thereby enabling it to report nearest neighbors incrementally. This process can be made more efficient by keeping $B$ in sorted order based on distance from $q$. However, this still leaves open the question of how to efficiently add and remove nodes from $B$.

Having made this modification, we can go even further and change the control structure. In particular, instead of keeping to the strict depth-first traversal, the list $B$ can be used to guide the traversal, i.e., the node in $B$ closest to $q$ is taken as the next node to process. As a node is processed, it is deleted from $B$, and as a non-leaf node is processed, all its entries are added to $B$. Note also that as described above, $B$ is sorted in MINDIST order. It could be ordered by MINMAXDIST, but such an ordering has the disadvantage that the node on $B$ nearest to $q$ would not be immediately accessible. Furthermore, we observe that the penalty for choosing to process a wrong node is far less than the penalty for doing so in the $k$-nearest algorithm since all that is done is to inspect the node's entries, rather than traversing its entire subtree (subject to pruning, of course).

Note that with this transformation it is now possible to allow an unbounded $k$, as the last element in NearestList, i.e., the one farthest from $q$, no longer plays a role. Of course, this also means that NearestList is no longer bounded, except by the total number of objects in the R-tree.

The entire process can be performed most easily by merging $B$ and NearestList into one list called CombinedNearestList. By ordering CombinedNearestList in increasing order of distance we are able to preserve the role of the previous contents of ActiveBranchList, in that nodes that would have been pruned will be at greater distances in the CombinedNearestList than the $k^{\text {th }}$ nearest object. Thus they and their subtrees will not be traversed when outputting the $k$ nearest neighbors. Observe that the transformed algorithm makes use only of the MiNDIST distance metric, thereby rendering moot the issue of whether or not to use the MiNMAXDIST [45] metric. Also, the transformed algorithm will in general achieve more pruning of nodes than the original $k$-nearest neighbor algorithm.

We conclude our discussion of the $k$-nearest neighbor algorithm by pointing out that the transformation yields an algorithm equivalent to the incremental algorithm presented earlier when CombinedNearestList is organized with a priority queue.

\section{Experimental Results}

In order to evaluate the R-tree incremental nearest neighbor algorithm of Figure 4 (denoted by INN), we compared it to the result of using the R-tree $k$-nearest neighbor algorithm of [45] (denoted by $k$-NN) for distance browsing (Section 6.1). We also measured the incremental cost of using INN, i.e., the cost of obtaining the $k+1^{\text {st }}$ neighbor once we have already obtained the $k^{\text {th }}$ neighbor (Section 6.2). By varying the number of objects that are browsed, we were able to see the true advantage of our method of computing the nearest neighbors incrementally rather than committing ourselves to a predetermined number of nearest neighbors, as would be the case if we used the $k$-nearest neighbor algorithm. (Remember that we do not know in advance how many objects will be browsed before finding the desired object.) Finally, we compare INN with 
$k-\mathrm{NN}$ for computing the result of a $k$-nearest neighbor query (Section 6.3). These studies were performed for small numbers of neighbors (i.e., less than 25), as this is the most common situation in which distance browsing is useful. Nevertheless, we also treat the case of a large number of neighbors in Section 6.3.

We measured the execution time as well as the disk I/O behavior for two different representative maps. In order to see whether the size of the maps was a factor we also performed a limited number of experiments in which the size was varied (Section 6.4). Our data consisted of line segments. We used both real-world data and randomly generated data. The real-world data consisted of four data sets from the TIGER/Line File [43] (see Figure 9):

1. Howard County: 17,421 line segments.

2. Water in the Washington DC metro area: 37,495 line segments.

3. Prince George's County: 59,551 line segments.

4. Roads in the Washington DC metro area: 200,482 line segments.

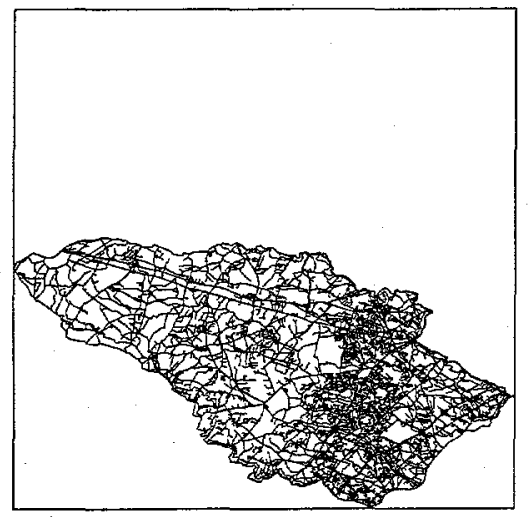

(a)

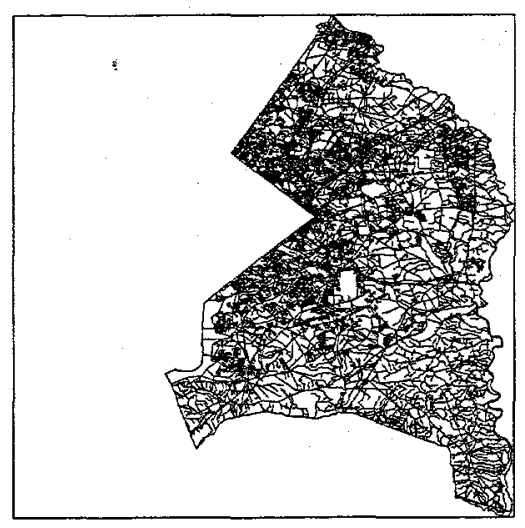

(c)

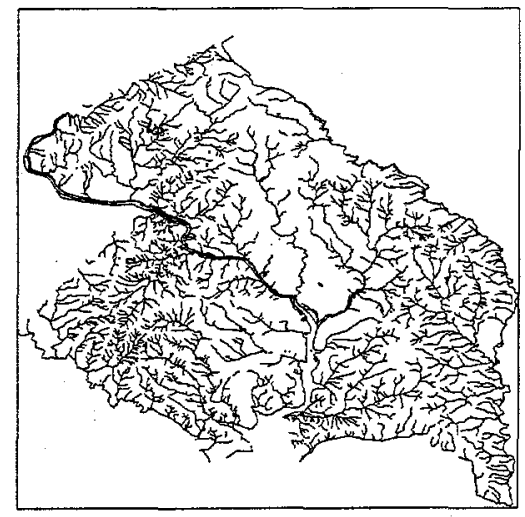

(b)

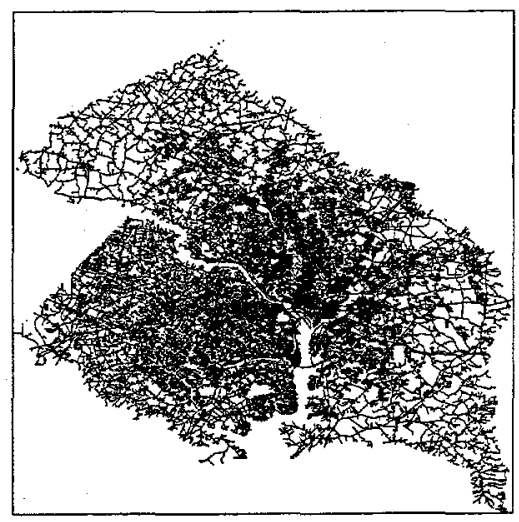

(d)

Figure 9: The four real-world data sets from the TIGER/Line File: (a) Howard, (b) Water, (c) PG, and (d) Roads. 
The randomly generated line segment maps were constructed by generating random infinite lines in a manner independent of translation and scaling of the coordinate system [38]. These lines were clipped to the map area to obtain line segments, and then subdivided further at their intersection points with other line segments so that at the end, line segments meet only at endpoints. Note that the random maps do not necessarily model real-world maps perfectly. In particular, by their construction, random maps cover an entire square area, whereas this is not the case for most real maps (e.g., TIGER/Line File county maps). Furthermore, the random maps tend to be rather uniform, while real maps tend to have dense clusters of small line segments mixed with more sparsely covered areas. Nevertheless, these randomly generated maps do capture some important features of real maps (e.g., there is a low probability of more than four line segments meeting at a point), and they enabled us to run the experiments on a wide range of map sizes for maps with similar characteristics.

Our experiments differ from those in [45], which used a Hilbert-packed R-tree [31,46], whereas we used an $\mathrm{R}^{*}$-tree. The Hilbert-packed R-tree is a static structure, constructed by applying a Peano-Hilbert space ordering (e.g., [47]) to spatial objects on the basis of their centroids. The leaf nodes of the R-tree are then built by filling them with the objects, and the non-leaf nodes are built on top, with bounding rectangles computed for the nodes. Notice that the conventional R-tree node splitting rules were not applied in the construction of the Hilbert-packed R-tree since each node is filled to capacity by the Hilbert-packed R-tree construction algorithm. As we are interested in dynamic environments we chose to use the $\mathrm{R}^{*}$-tree rather than the Hilbertpacked R-tree for our experiments except where noted.

Most of the data sets that we used were small enough to fit in main memory of many modern computers (except in the experiments reported in Section 6.5). Nevertheless, we used a disk-based R-tree structure, and employed buffers to store a limited number of recently used R-tree nodes (128). We therefore believe that our results will scale well to large data sets. The fact that we employ buffered $\mathrm{V} / \mathrm{O}$, with the added possibility of a requested disk block being in a disk cache or in operating system buffers, complicates the comparison between the two algorithms. There are two extremes: for each $1 / O$, the requested disk block is found in memory, or every I/O leads to disk activity. Given a query for a fixed number of neighbors, the incremental nearest neighbor (INN) algorithm shows less improvement over the $k$-nearest neighbor algorithm ( $k$-NN) in the former case (i.e., if the entire data sets resides in memory), and may even be slower, as will be seen for the small random data sets. This is mainly due to the overhead incurred by priority queue operations. However, for the other extreme the INN algorithm would show even more advantage than we found, as it always requests fewer R-tree nodes and objects than the $k$-NN algorithm.

For each experiment, we ran multiple queries on the same data set for the same number of neighbors. This was done so that more than one query point could be tested, as well as to make sure that the timing results were meaningful (given the timing granularity of the system we used). Since our R-tree implementation utilizes buffered $\mathrm{V} / \mathrm{O}$, this means that a query may access disk blocks that have already been loaded into the buffer by earlier queries in the same sequence. We feel that this was a reasonable choice to make, since the buffers were small compared to the data size, and clearing them prior to each query would have affected the timing results. Also, in a real world scenario, it is likely that a user will execute more than one query for a given map.

We use three measures for comparing the algorithms: execution time, R-tree node I/O (frequently referred to as disk $V O[6,32]$ ), and object distance calculations. The R-tree node $\mathrm{I} / \mathrm{O}$ is reported as the number of accesses, and may not correspond to actual disk $\mathrm{I} / \mathrm{O}$ if nodes can be found in database or system buffers. However, we have found that the number of accesses predicts the relative performance of actual disk L/O reasonably well. Furthermore, any saving due to buffering will show up in reduced execution time. Thus we used the disk I/O characterization. 
In all the experiments that we conducted, the maps were embedded in a $16 \mathrm{~K}$ by $16 \mathrm{~K}$ grid, and the capacity of each R-tree node was 50. In order to simplify the analysis of the execution time results, we chose to store the actual line segments in the R-tree leaf nodes instead of just their bounding boxes. Also, the organization of the external object storage has a large effect on the performance, and thus introduces an extra variable into the comparison of the two algorithms. Query points were uniformly distributed over the space covered by the map data, and the distance functions used to measure the distances of lines and bounding rectangles from the query points were based on the squared Euclidean metric (in order to avoid computing square roots). The experiments were run sufficiently often to obtain consistent results with a different query point each time. Execution times are reported in milliseconds per query; they include the CPU time consumed by the algorithm and its system calls. We used a SPARCstation 5 Model 70 rated at 60 SPECint 92 and 47 SPECfp 92 , and a GNU $\mathrm{C}++$ compiler set for maximum optimization $(-\mathrm{O} 3)$.

\subsection{Cumulative Cost of Distance Browsing}

In this section we focus on the distance browsing query when we do not know in advance how many neighbors will be needed before the query terminates. In this case, we need to reapply the $k$-nearest neighbor algorithm as the value of $k$ changes. In contrast, in the case of the incremental nearest neighbor algorithm, we need to reinvoke the algorithm to obtain just one neighbor (i.e., the next nearest one). For these experiments we used the map of Prince George's County (denoted by $P G$ in the figures) as well as a randomly generated line map of a similar size, containing 64,000 lines (denoted by $R 64 K$ ). We included the random line map to see if the performance was affected by some unknown characteristics of the PG map.

Figures 10 through 12 show each measure's cumulative cost for distance browsing through the database by finding the neighbors incrementally. There are a number of ways of using a $k$-nearest neighbor algorithm to perform distance browsing. In our tests (shown in the figures) we use two such methods: (1) Execute $k$-NN each time we need a new neighbor. (2) Invoke $k$-NN for every five neighbors. Thus, for example, in case (2) the cost of computing the $11^{\text {th }}$ through $14^{\text {th }}$ neighbors is the same as the cost of computing the $15^{\text {th }}$ neighbor (which requires invoking the $k-\mathrm{NN}$ algorithm for $k=5,10$, and 15). From the figures, it is clear that using the incremental nearest neighbor (INN) algorithm for distance browsing significantly outperforms simulating incremental access with the $k$-NN algorithm. In fact, the difference quickly becomes an order of magnitude. The figures use a logarithmic scale for the $y$-axis in order to bring out relative scale. Since the differences were so great, in order to simplify the presentation, we include results only for the $k$-NN algorithm for the PG map, as the results for the random data were similar.

The method that we used above for choosing the value of $k$ when performing distance browsing with the $k$-NN algorithm is not the best that we can do for larger values of $k$. For example, it would be better to multiply $k$ by 2 each time the algorithm must be re-invoked. In addition, the $k$-NN algorithm can be adapted to make it more suitable for use in distance browsing. In particular, after finding the $m$ nearest neighbors and determining that we must find the $m^{\prime}>m$ nearest neighbors, we can use the distance of the $m^{\text {th }}$ nearest neighbor as a minimum distance when the $k-\mathrm{NN}$ algorithm is re-invoked with $k=m^{\prime}$ (actually, $k$ is set to $m^{\prime}-m$, since the $m$ nearest neighbors would be excluded from the search). This minimum distance can be used to prune the search in much the same way as we described using minimum distance in the INN algorithm in Section 4.5. Some complications arise if other objects have the same distance from $q$ as the $m^{\text {th }}$ nearest neighbor. The best way to resolve this is to return all neighbors with that distance, which means that sometimes we obtain more neighbors than we requested. In Figure 13 we compare the execution time when using such an adapted $k$-NN algorithm (labelled "Prune") for distance browsing to the execution time when using the INN algorithm. Also, we show the result for the unmodified algorithm, where we must restart the search from scratch when the $k$-NN algorithm must be re-invoked (labelled "Restart"). We show the results 


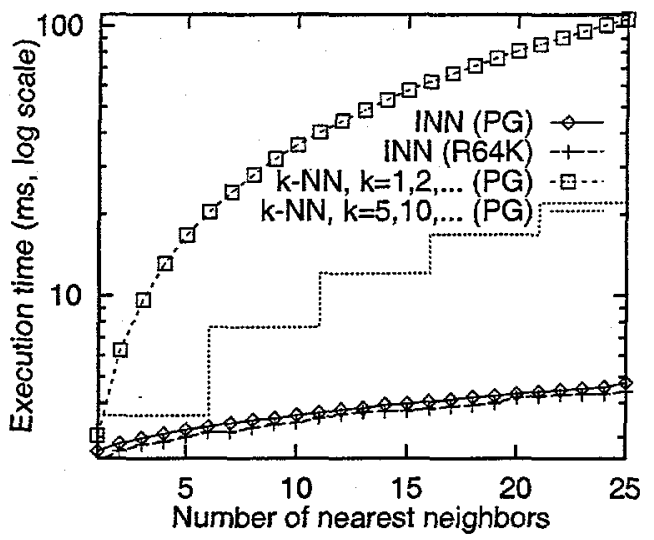

Figure 10: Cumulative execution time for distance browsing.

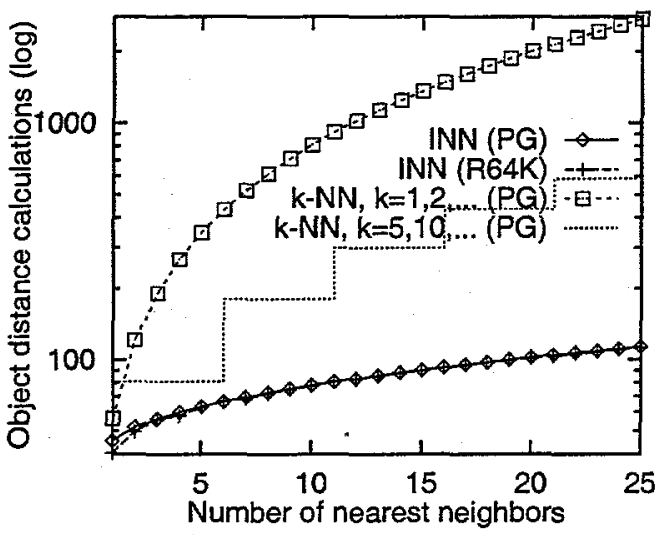

Figure 12: Cumulative object distance calculations for distance browsing.

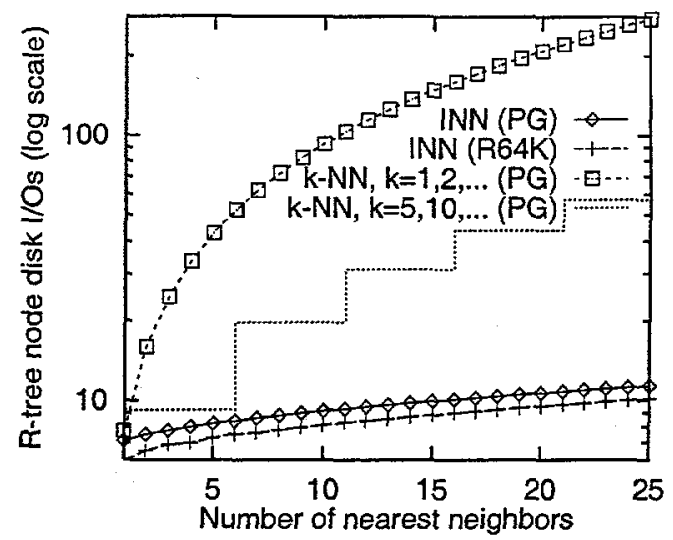

Figure 11: Cumulative R-tree node disk $\mathrm{l} / \mathrm{O}$ for distance browsing.

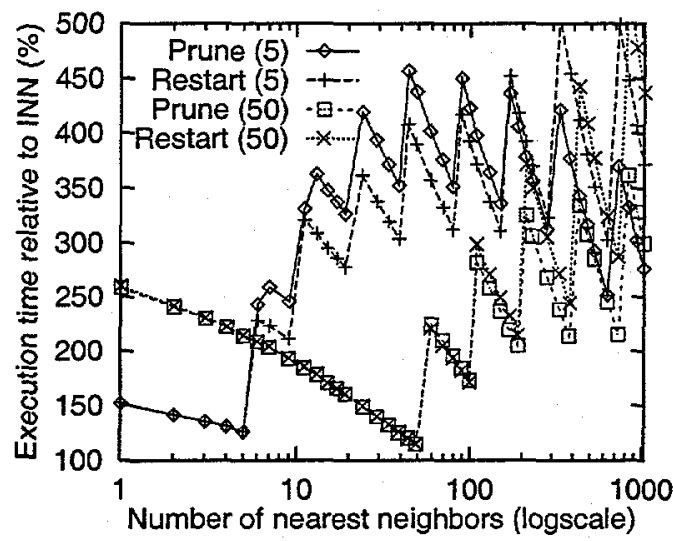

Figure 13: Execution time of $k$-NN relative to that of INN when used for distance browsing when the $k$-NN approach is made as good as possible.

only for the real-world data set (PG), as they were almost identical when using the random data set.

We use two different starting values for $k$ in Figure 13, namely 5 and 50 (shown in parentheses). Each time the $k$-NN algorithm is re-invoked, $k$ is doubled. The figure shows that if the $k$-NN algorithm must be re-invoked at least once, it usually takes more than twice (and up to nearly five times) as long as the INN algorithm. Using the "Prune" variant of the $k$-NN algorithm does not pay off unless a rather large number of neighbors is needed (over 100 or 200 in these experiments). The reason why this variant takes longer for a smaller number of neighbors is that not enough nodes get pruned to offset the cost of more node distance computations (for each node we must compute two distances, a minimum and a maximum, instead of just the minimum). Another observation is that the the $k$-NN approach is highly sensitive to the initial value of $k$, and which initial value is better depends on how many neighbors we need (which we do not know in advance in distance browsing). The spikes on the curves occur where the $k$-NN algorithm is re-invoked an additional time for higher values of $k$, and between a spike and the next low point, no more neighbors are computed ${ }^{4}$.

\footnotetext{
${ }^{4}$ There should be a spike at 5 neighbors for "Prune (5)", but instead it occurs at 6 neighbors. The reason for this is that occasionally when requesting the nearest five neighbors, the sixth nearest neighbor has the same distance as the fifth one, so the $k$-NN algorithm
} 
The reason the slope of the curve decreases after each spike is that in the range from a spike to the next low point, the cost of the $k$-NN approach remains constant (since no more neighbors are computed) while the cost of the INN approach increases gradually as we must compute additional neighbors. Note that the absolute low point on the two curves corresponds to the case where the number of neighbors needed happens to be equal to the initial value of $k$ ( 5 and 50, respectively). For those values of $k$, the $k$-NN algorithm is not much slower than the INN algorithm (about 25\% slower for $k=5$ and $14 \%$ slower for $k=50$ ).

\subsection{Incremental Cost of Distance Browsing}

The results of the experiments conducted in Section 6.1 show the total cost of distance browsing after retrieving the $k^{\text {th }}$ neighbor. Using INN to implement each browsing step requires us to examine just one neighbor regardless of how many browsing steps we have already executed. In contrast, use of $k$-NN for distance browsing requires us to examine $k+1$ neighbors when $k$ browsing steps have already been executed. In this section, we compare the two algorithms in terms of the cost of each browsing step (i.e., the incremental cost). This is shown in Figures 14 through 16. For the INN algorithm, the incremental cost can be seen to fluctuate somewhat, but it is always at least one order of magnitude less than the cost of the $k$-NN algorithm once the first neighbor has been obtained. Although not shown here, we found this to hold for all values of $k$. Again, we use a logarithmic scale for the $y$-axis so that the fluctuation in the cost of the incremental algorithm can be seen more clearly.

We evaluated the incremental execution time for up to 1000 neighbors in the PG map. Interestingly, we found that the incremental execution time clusters around an average of about $.04 \mathrm{~ms}$ after the first 100 neighbors or so. This is in agreement with the results that we discuss in Section 6.3, where we find that the average execution time per neighbor is around $.04 \mathrm{~ms}$ when retrieving a few thousand neighbors or more in the PG and R64K maps. Thus we see that for a given map, the incremental execution time is remarkably close to constant after a small fraction of the objects have been retrieved (for the PG map this was around 100 neighbors or less than $0.2 \%$ of the map size).

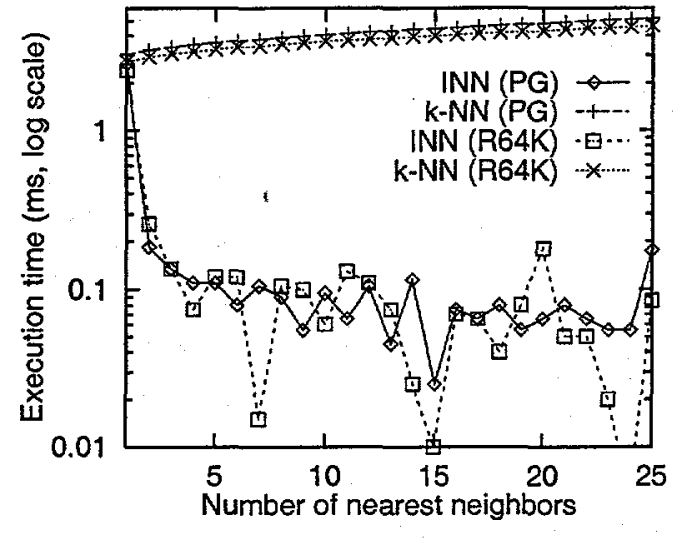

Figure 14: Incremental execution times for distance browsing.

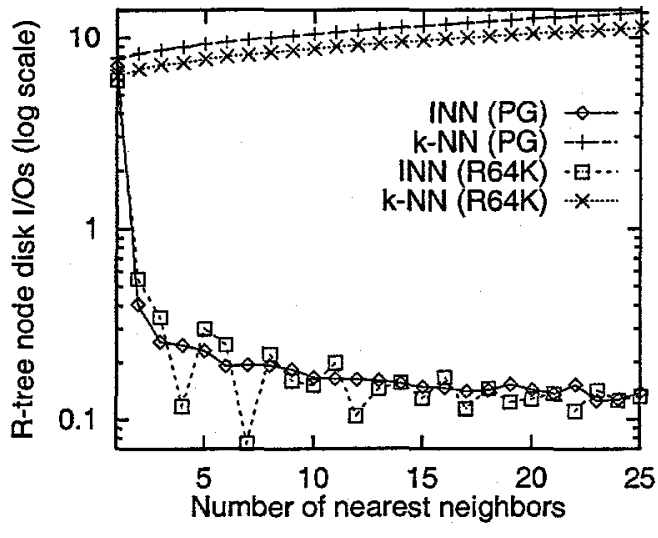

Figure 15: Incremental R-tree node disk $\mathrm{l} / \mathrm{O}$ for distance browsing.

For the R-tree node disk I/Os (Figure 15), the incremental algorithm (INN) was at least an order of magnitude better than $k$-NN after the first neighbor had been found. INN appears to be decreasing (i.e., between .1 and .2 after 25 neighbors), but levels off after a few hundred neighbors have been found. (The graph is not 
a step function because the number of node accesses is averaged over many queries.)

For the object distance calculations (Figure 16), the incremental algorithm (INN) was at least an order of magnitude better than $k$-NN after the first few neighbors had been found. The improvement approaches two orders of magnitude when 25 neighbors have been found, and continues in this manner for larger values of $k$ (not shown here). The average number of distance calculations performed for each incremental invocation is seen to be decreasing. This continues as more neighbors are retrieved and is below 1.2 after 300 neighbors. Thus INN quickly reaches a stage of accessing only about one object per reported neighbor.

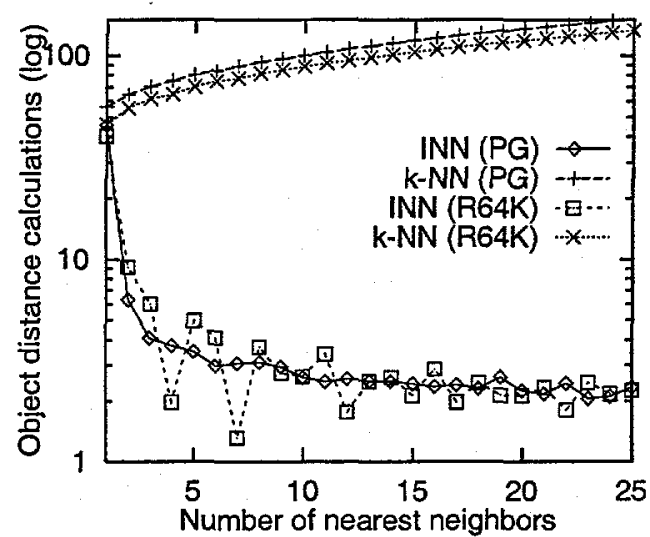

Figure 16: Incremental object distance calculations for distance browsing.

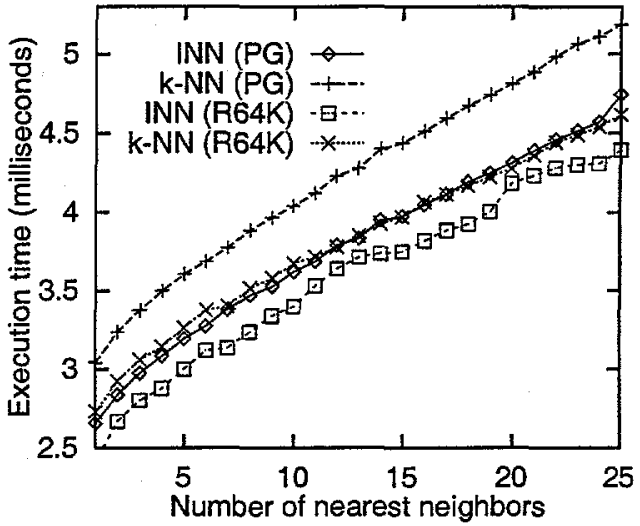

Figure 17: Execution time for $k$ nearest neighbor query.

\section{$6.3 k$-Nearest Neighbor Queries}

We now consider what the cost would be if we used the incremental nearest neighbor algorithm to solve the $k$ nearest neighbor problem. In other words, instead of browsing the database on the basis of distance, obtaining one neighbor at a time, we address the related problem of finding all $k$ neighbors at once, as we would do if we knew in advance how many neighbors we need. It is interesting to see if a performance penalty is incurred in solving this classical problem by using our incremental algorithm, rather than using approaches such as the $k$-NN algorithm which obtain all $k$ neighbors at once. We ran a sequence of tests in the same manner as those reported in Sections 6.1 and 6.2; the results are shown in Figures 17 through 19. From these figures we observe that using the INN algorithm leads to no sacrifice of performance. In fact, the incremental algorithm outperforms the $k$-nearest neighbor algorithm for the two maps for all values of $k$.

In addition to the experiments mentioned above, we ran $k$-nearest neighbor queries for values of $k$ from 1 up to the size of the data set. The results of these experiments are reported in Figures 20 through 22, where the cost measures are divided by the number $k$ of nearest neighbors, so that we are reporting the cost per neighbor. For the incremental nearest neighbor algorithm, this value is close to the average incremental cost for all but the smallest values of $k$ (for small $k$, the cost of retrieving the first neighbor dominates the cost). Dividing the cost measures by $k$ makes it possible to distinguish the cost measures for large values of $k$, which is difficult otherwise. In Figures 20-22, the $y$ axis uses a logarithmic scale.

For the execution time (Figure 17), we see that the two algorithms have similar growth patterns, with $k$ NN being somewhat slower than INN (about $11-14 \%$ for PG and 4-10\% for R64K). While the improvement of INN over $k$-NN is modest for values of $k$ up to 25 , Figure 20 reveals that the difference widens as $k$ grows larger, up to $75 \%$ for $\mathrm{PG}$ and $87 \%$ for R64K (for $k=2^{15}=32,768$ ). Even for values of $k$ as small as 
several hundred, the improvement of INN over $k-\mathrm{NN}$ is $20-30 \%$. Note how the performance of INN for the two maps is very similar, whereas the performance of $k-\mathrm{NN}$ is worse for the PG map than for the R64K map. This observation holds for the other two cost measures as well. This suggests that INN is much less sensitive than $k$-NN to the distribution of data objects.

For very large values of $k$, we may ask whether it is not better to simply calculate distances for the entire database and then sort on the distance. If all the objects are ranked with the INN algorithm (or the $k$-NN algorithm), we must also compute the distances for all the objects in the database. The question then reduces to whether the overhead of the INN algorithm (for computing distances of nodes and manipulating the priority queue) exceeds the cost of sorting all the distance values once they have been computed. Interestingly, we found that for the PG map, using the INN algorithm to rank all the objects was faster than computing all the distances and sorting them, whereas the $k-\mathrm{NN}$ algorithm was a little slower than the sorting approach. Of course, this result cannot be generalized, as it depends on numerous factors, such as size of the data set, the spatial index being used, and whether the spatial objects are stored directly in the leaf nodes of the R-tree or in an external object table.

For the R-tree node disk I/Os (Figure 18) we see that INN is always better than $k-\mathrm{NN}$, while the rate of growth is similar for both and appears to be linear in $k$ for low values of $k$. In fact, we found that this same pattern held for all values of $k$, as we see in Figure 21. The figures show that for each value of $k$, INN achieves more pruning of the input tree than $k$-NN. This partially explains its better execution time performance. For values of $k$ ranging between $2^{6}$ and $2^{15}$, INN accesses $20-53 \%$ fewer nodes for PG and $12-35 \%$ for R64K, with the largest difference occurring at $2^{9}$ for both maps.

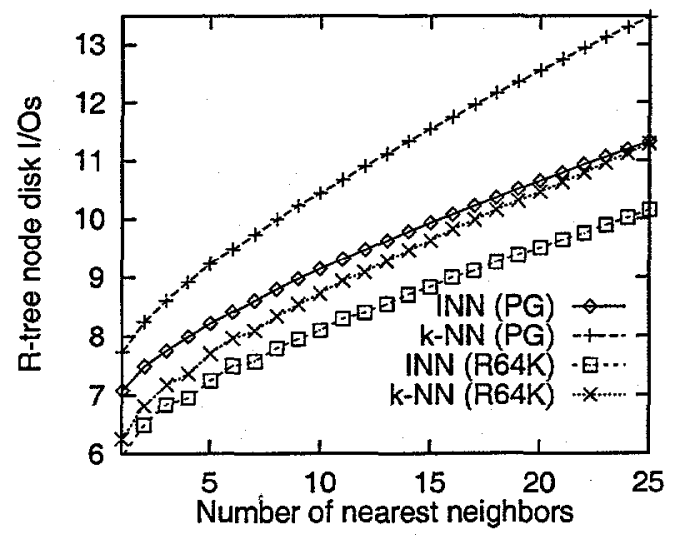

Figure 18: R-tree node disk $\mathrm{I} / \mathrm{O}$ for $k$ nearest neighbor query.

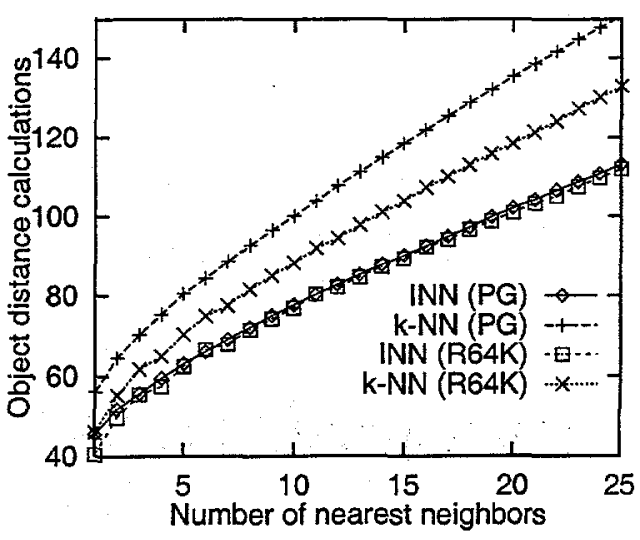

Figure 19: Object distance calculations for $k$-nearest neighbor query.

For the object distance calculations (Figure 19), we see that the INN algorithm again outperforms the $k$ NN algorithm. Figure 22 shows that this holds for all values of $k$, except when ranking all the map objects (in which case the number of distance calculations equals the number of map objects in both cases, as no pruning of objects or nodes is possible). The shapes of the curves in Figure 22 can be seen to be very similar to those in Figure 21. This is not surprising when we realize that the number of distance calculations is proportional to the number of R-tree leaf nodes that are accessed, and the leaf nodes in an R-tree greatly outnumber the non-leaf nodes.

Figure 23 shows the fraction of total execution time that is attributed to disk I/O operations in the above experiments. We compute this by recording the node accesses performed during the execution of the algorithms, and measuring the time needed to do nothing but access those nodes. The figure shows that the 


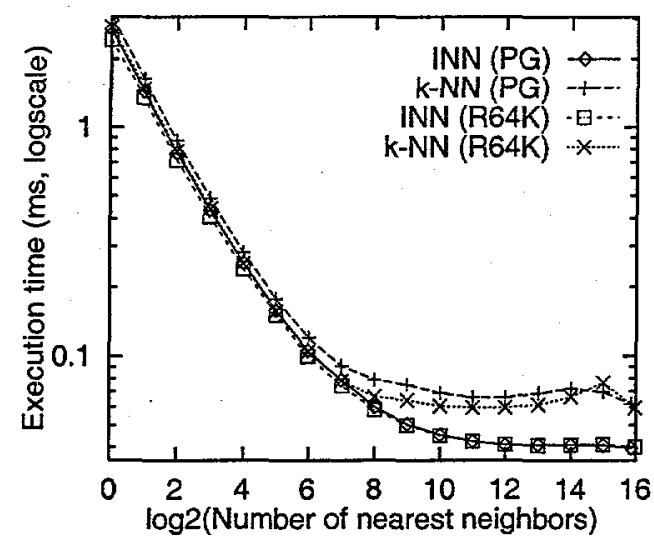

Figure 20: Execution time per neighbor for $k$-nearest neighbor query.

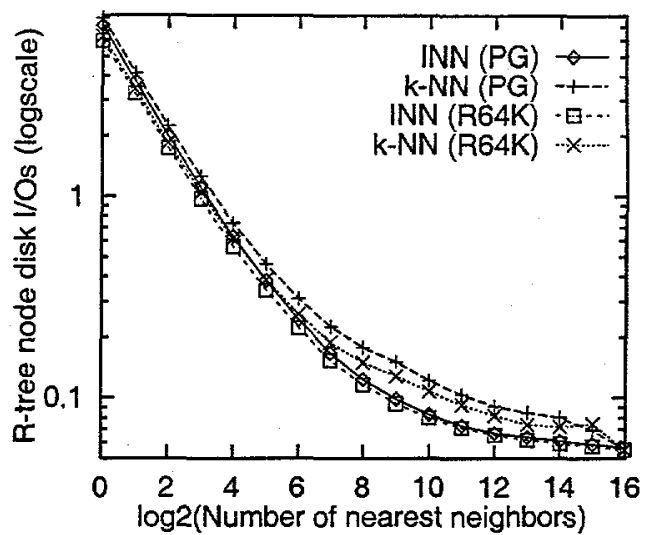

Figure 21: R-tree node disk $\mathrm{l} / \mathrm{O}$ per neighbor for $k$-nearest neighbor query.

fraction of time spent by the INN algorithm in doing I/O is relatively constant, but starts to decrease for a large number of neighbors. In contrast, the fraction of time spent by the $k$-NN algorithm in doing $\mathrm{V} / \mathrm{O}$ has a much larger variation, initially increasing rapidly, and decreasing significantly as the number of neighbors needed increases. In fact, eventually the fraction of time spent in doing I/O by the $k$-NN algorithm is considerably less than that spent by the INN algorithm as the number of neighbors increases; thus the INN algorithm becomes more efficient from a CPU cost perspective. (This may be due, in part, to the fact that for a large number of neighbors, the priority queue for the INN algorithm is considerably smaller than the NearestList maintained by the $k$-NN algorithm, as discussed in Section 6.6 and seen in Figure 31.)

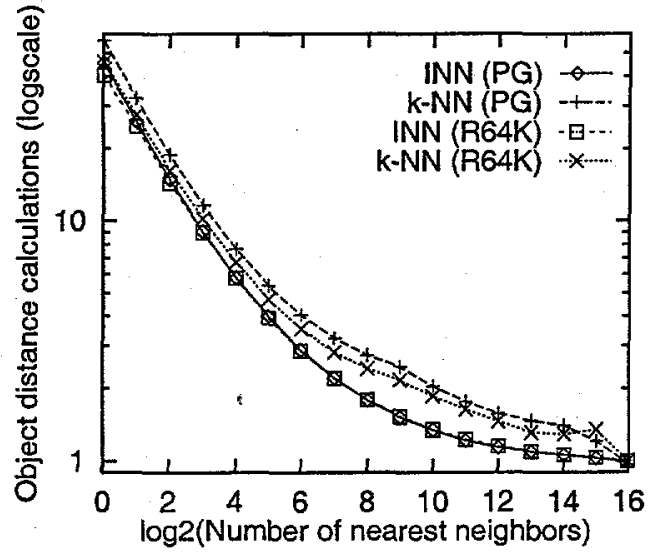

Figure 22: Object distance calculations per neighbor for $k$-nearest neighbor query.

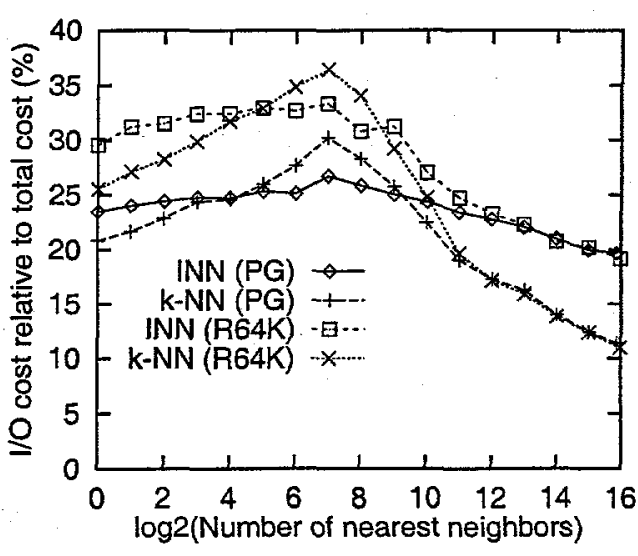

Figure 23: Fraction of total execution time taken by disk $/ / O$ s in computing $k$ nearest neighbor query.

\subsection{Results for Varying Data Size}

In the previous sections we investigated the performance of the two algorithms by varying the number of neighbors for both distance browsing and computing the $k$ nearest neighbors for similarly sized data sets. It is important that the performance of the algorithms remain reasonable even when the size of the data set is increased. To verify that this is indeed the case, we tested the performance of INN and $k$-NN on both random 
and real-world map data. Our experiments showed the same relationships for the two algorithms between the cumulative and incremental costs of distance browsing, as well as computing the $k$ nearest neighbors, that we found in the experiments reported in Sections 6.1-6.3 (provided the maps are non-trivial in size). In particular, they confirmed the superiority of INN over $k$-NN. In the interest of saving space we do not show these results here.

In the rest of this section, we focus on the relative behavior of the algorithms when finding the nearest neighbor (i.e., $k=1$ ). This operation is important as it is the first step in distance browsing, and as we saw in Section 6.2 its execution time dominates the cost of distance browsing for small values of $k$.

Figures 24 through 26 show the performance of the two algorithms when finding the nearest neighbor. The $x$-axis in the figure is $\log _{2} N$, where $N$ is the number of line segments. The real-world maps appear in the same order that we described them (from left to right: Howard County, Water, Prince George's County, and Roads). The random maps that we tested contained 1000, 2000, 4000, 8000, 16000, 32000, 64000, 128000 and 256000 line segments.

For the execution time (Figure 24), we see that the INN algorithm is faster for most of the maps; $k$-NN took from $10-19 \%$ more time for the real-world maps, and up to $14 \%$ more time for the randomly generated maps. The exceptions are the three smallest randomly generated maps. This can be explained partly by the fact that these maps were small enough to fit in the R-tree node buffer, and partly by the fact that their small sizes gave less room for improvement (see Figures 25 and 26). Even so, for larger values of $k$, INN became better than $k$-NN for these data sets. For all the randomly generated maps, which have similar characteristics, the rate of growth of the execution time can be seen to be nearly identical for the two algorithms. In fact, the rate of growth appears to be very nearly logarithmic in the number of line segments (recall that the $x$-axis uses a $\log$ scale). The execution times for the real-world maps correlate remarkably well with the execution times for the random maps of comparable size.

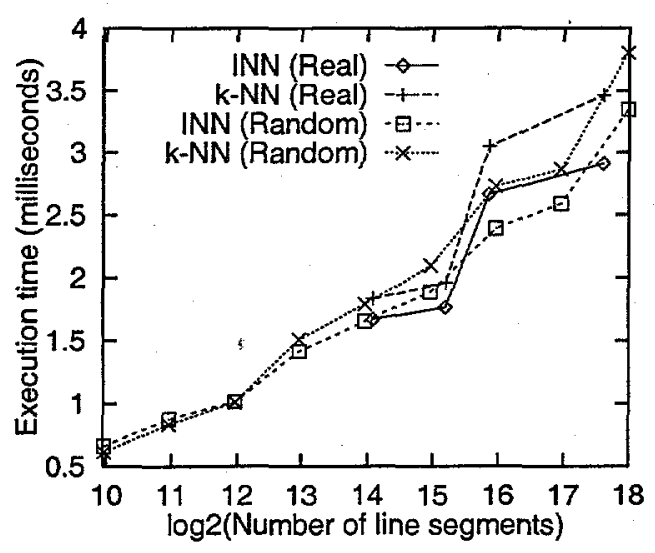

Figure 24: Execution time for finding one neighbor.

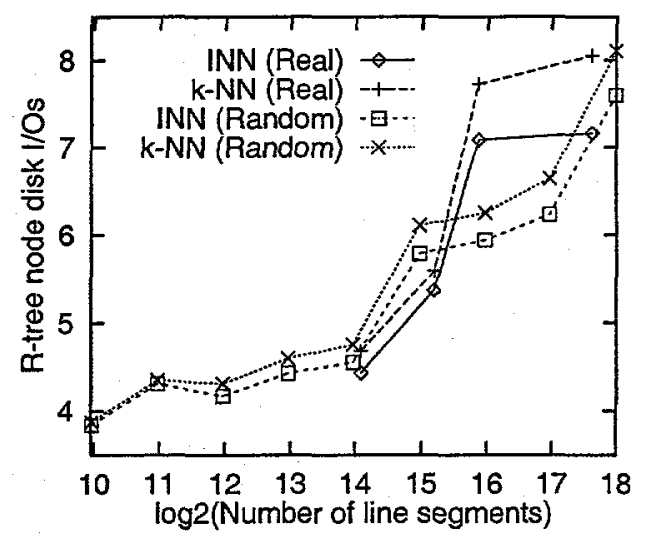

Figure 25: R-tree node disk $1 / 0$ for finding one neighbor.

For the R-tree node disk I/Os (Figure 25) we find the same relative behavior of the algorithms, with INN being always better than $k$-NN, while the rate of growth is similar for both. The rate of growth appears to be logarithmic in the number of line segments. This compares with the results reported in [45] for $k$-NN, where it was observed that the number of R-tree node accesses grew linearly with the height of the tree. Our experiments are not in exact agreement with that observation, but asymptotically, the two observations are equivalent, since in R-trees the height of the tree grows logarithmically with the number of objects.

For the object distance calculations (Figure 26), again, INN performs better than $k$-NN. 


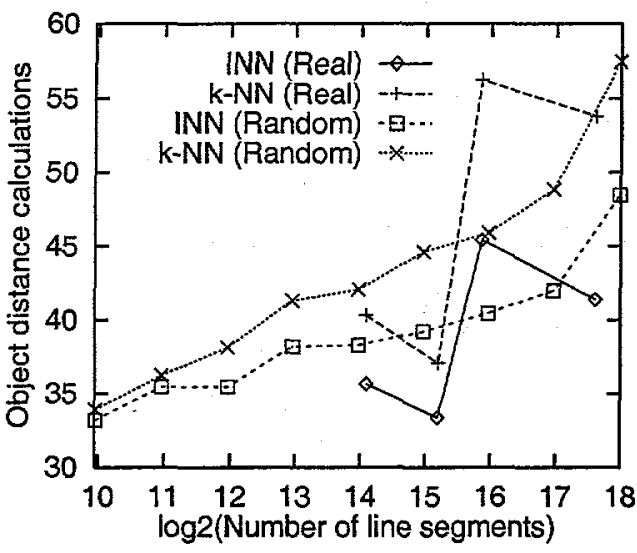

Figure 26: Object distance calculations for finding one neighbor.

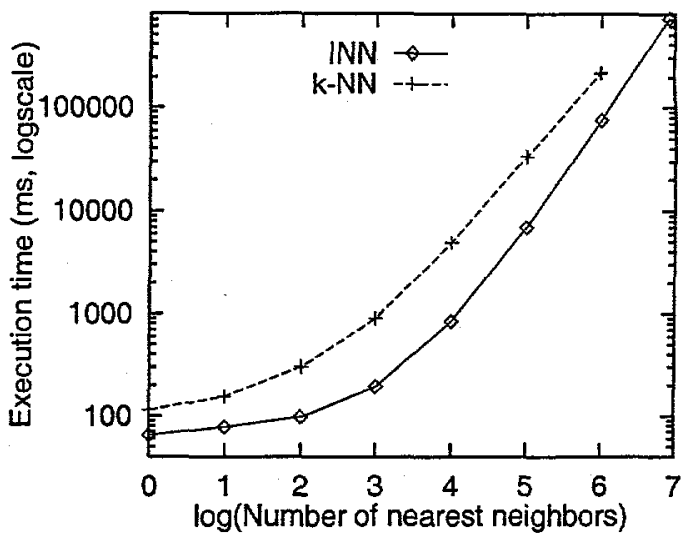

Figure 27: Execution time for large data set.

\subsection{Results for Large Data Sets}

Admittedly, the data sets that we used in the experiments reported above were moderate in size. For the largest data set that we used, the spatial index occupies approximately $9 \mathrm{MB}$ of disk space, which is small enough to fit into the main memory of most modern computers. Even so, in our experiments, we only used a small amount of main memory for buffers (128 nodes), and the size of the priority queue remained small compared to the data size (100 KB in the worst case for the experiments in Section 6.3 , or about $3 \%$ of the size of the map files). Thus we believe that our results will also hold for larger data sets, i.e., data sets much larger than the size of main memory.

In order to verify this claim, we conducted an experiment with a randomly generated data set of 8 million lines. As it was prohibitively slow to build an $\mathrm{R}^{*}$-tree for such a large data set, we built instead a Hilbertpacked R-tree [31], which occupied almost $300 \mathrm{MB}$. We used the same level of fan-out (50) and the same amount of buffering (128 nodes) as in our previous experiments (though it might have been better to use a larger fan-out and buffer sizes for such a large data set). [Incidentally, we found that both algorithms performed more poorly with a Hilbert-packed $\mathrm{R}$-tree than with an $\mathrm{R}^{*}$-tree for the same data set. This appears to be due to the greater amount of node overlap in the Hilbert-packed R-tree. The incremental nearest neighbor algorithm proved to be much less sensitive to the level of node overlap, due to its superior pruning of the R-tree nodes.]

Figures 27-29 show the results of our experiments on this large map, which consisted of $k$-nearest neighbor queries for values of $k$ from 1 through the size of the data set ( 8 million). Unfortunately, we were not able to run the $k$-NN algorithm for $k=8$ million, as there was not enough memory to hold the neighbor list for 8 million neighbors. This is in contrast to the INN algorithm, where the priority queue contained at most about 83,000 elements, or about $1 \%$ of the number of neighbors. The speedup in execution time for INN over $k$-NN ranged from 1.8 to 5.8 . $k$-NN accessed from 1.8 to 5.3 times as many nodes and performed up to 6 times as many distance calculations as INN.

\subsection{Priority Queue Size}

In Section 4.8 we showed that in the worst case, all the data objects must be inserted into the priority queue when using the incremental nearest neighbor algorithm. In our experiments, however, we found that the 
To appear in ACM Transactions on Database Systems (also Univ. of MD Computer Science TR 3919)

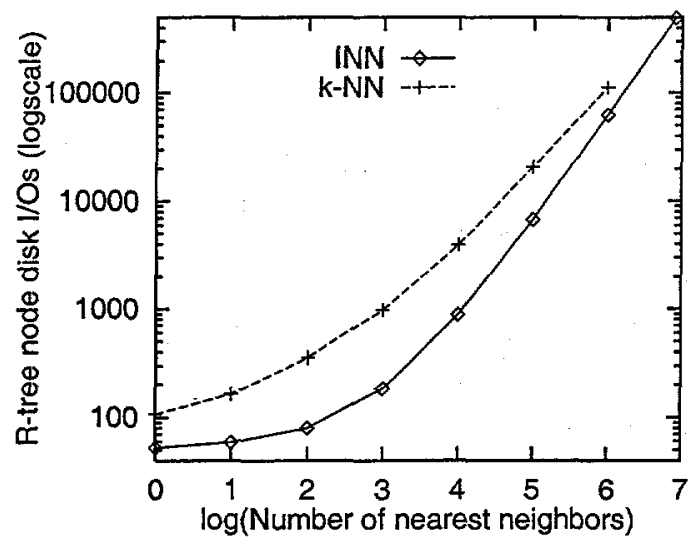

Figure 28: Node disk I/Os for large data set.

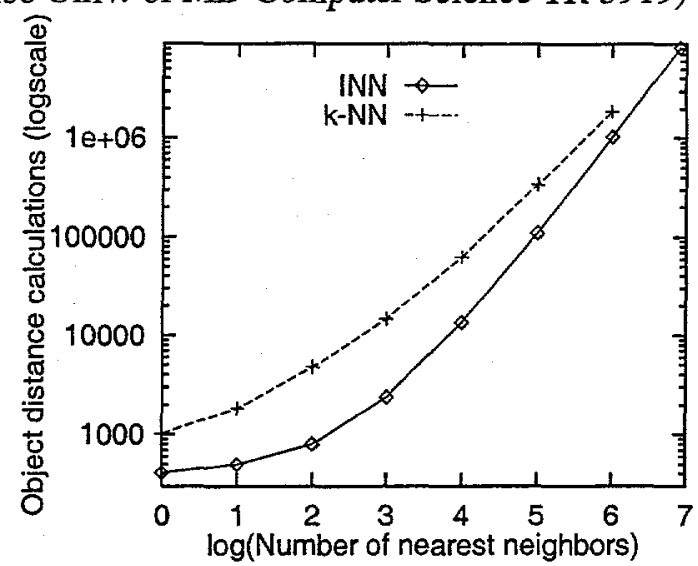

Figure 29: Object distance calculations for large data set.

priority queue remained modest in size. The size of the priority queue affects the performance of queue operations during the algorithm's execution. Also, a very large queue requires a disk-based implementation, thereby slowing the algorithm down. However, in most applications the maximum queue size remains relatively modest, which permits using a memory-based data structure for the queue. For example, consider Figure 30, which shows the maximum size of the queue when computing the nearest neighbor (i.e., $k=1$ ) using the same data sets as in Section 6.4. Notice that for the worst case situation described above, in this first step of distance browsing for the given query object, all objects must be inserted into the queue before determining the nearest neighbor. From the figure it is evident that the maximum queue size grows remarkably slowly as the number of line segments increases. The results for the random maps suggest that this growth is logarithmic in the number of line segments.

Figure 31 shows the maximum size of the priority queue when using the incremental nearest neighbor algorithm after $k$ distance browsing operations for the maps used in Section 6.1 ( $k$ ranged from 1 up to the size of the map). In the figure, the $y$-axis is logarithmic. We see that the maximum queue size $M$ grows extremely slowly. Note also that $M$ is relatively small (less than $5 \%$ in the worse case) in comparison with the sum of the number of data objects and R-tree nodes for the two comparably-sized maps, which is $M$ 's theoretical maximum. When $k$ reaches a value of $2^{10} \approx 1000$, the priority queue needed by the incremental nearest neighbor algorithm is smaller than the priority queue needed to store the sorted buffer for the $k$-NN algorithm. A similar picture emerged for the large map used in Section 6.5, where the size of the priority queue was an even smaller fraction of the map size (1\% in the worst case).

\section{High-Dimensional Space}

As already pointed out, the incremental nearest neighbor algorithm is independent of the dimensionality of the data objects and is equally applicable to data embedded in low-dimensional and high-dimensional spaces. Unfortunately, it is difficult to effectively index high-dimensional data and nearest neighbor search also becomes more costly. In this section we address some of the issues that arise. As it is hard to reason about arbitrary data distributions, some of the conclusions we draw are based on uniformly-distributed data.

High-dimensional data arises in a number of current applications, including multimedia databases, data warehouses, and information retrieval. Usually, such data is limited to points, but more general objects also arise [8]. As an example of an application that leads to high-dimensional data, color histograms have been 


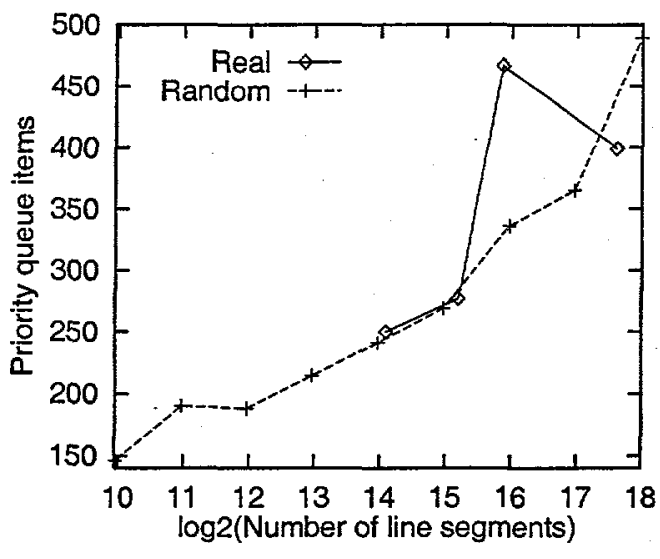

Figure 30: Maximum queue size for finding the nearest neighbor (i.e., $k=$ 1).

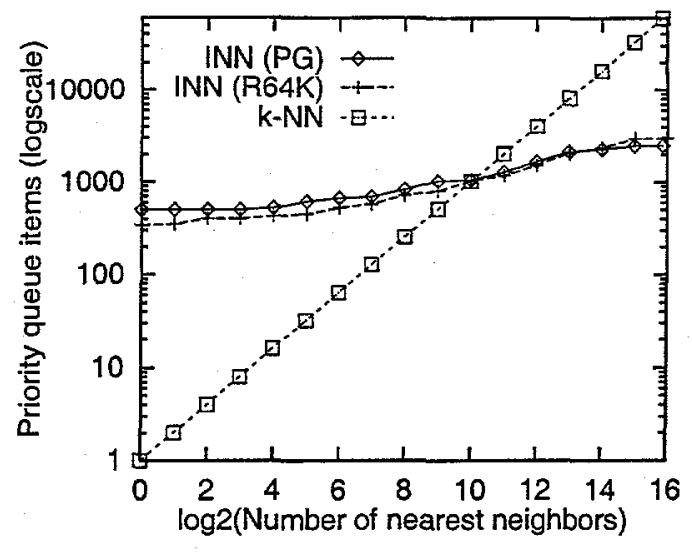

Figure 31: Maximum queue size for a wide range of $k$.

used in image databases to allow searching for images with a specific color or with a combination of colors similar to some query image. The colors in an image are described by $d$-dimensional vectors, in which each element encodes the intensity of a particular range of colors (e.g., by using RGB values). To compare the closeness of the sets of colors in two images, a complex distance function is used, involving matrix multiplication. Using that distance function, we can use a nearest neighbor search on an image database to find the image closest in color to some query image. The number of dimensions, $d$, for color histograms is typically 64,100 or 256 . In other applications, the number of dimensions can be even higher (as much as several thousand).

Most spatial indexing structures do not work very well for high dimensions. The R-tree, for example, has been found to degenerate for dimensions higher than 7 or so [9]. Specifically, what happens is that even for range queries with small query windows, so many of the index pages must be read that reading them is more expensive than sequential scan of the data. Several indexing structures have been proposed to address this issue; for example the X-tree [9] and $\mathrm{LSD}^{\mathrm{h}}$-tree [27], based on the R-tree and LSD-tree, respectively. However, even these often do not provide much speedup compared to sequential scan for dimensions above 20 or so. An approach often taken to speed up access to point data of very high dimension is to map the points into a space of lower dimension [18,34], in which case we can use the incremental nearest neighbor algorithm on the lower-dimensional space. In order to guarantee the accuracy of the result, the output of the algorithm can be filtered based on the distances of the corresponding higher-dimensional points [48]. Another approach is to abandon the goal of indexing the data points based on space occupancy and instead use properties of the distance metric employed (see the discussion of the metric space model in Section 2). If a hierararchical index method based on distance (e.g., $[11,14,52]$ ) is employed, our algorithm is still applicable. In fact, the $k$-nearest neighbor algorithm presented in [14] is similar to our algorithm in that it uses a priority queue for nodes to guide the traversal of the index.

If we use the Euclidean distance metric, the nearest neighbor search region (Section 4.6) is spherical. On the other hand, the node regions for most types of spatial index structures are hyper-rectangular in shape. This has the effect of making nearest neighbor search more expensive, as more points are accessed than necessary. To see why this is true, consider that in two dimensions the areas of a square and a circle, both with radius $r$, are $4 r^{2}$ and $\pi r^{2}$, respectively. Thus the ratio of the area of the circle to the area of the square is $\pi / 4 \approx 79 \%$. In three dimensions the ratio of the volume of a sphere to the volume of a cube is about $52 \%$, and in four dimensions the corresponding ratio for a hypersphere and hypercube is $10 \%$. In general, the ra- 
tio between the volume of a hypersphere and its circumscribed hypercube decreases exponentially with the number of dimensions. Intuitively, the reason for this is that the number of "corners" of the hypercube grows exponentially with dimension. This effect has a direct consequence for nearest neighbor search using the Euclidean distance metric. To see why, let us assume that we have uniformly-distributed data points inside a hypercube of radius $r$ and a search region of radius $r$ centered inside the hypercube; the hypercube represents the smallest bounding box of the set of hyper-rectangular leaf node regions that intersect the search region. Then the proportion of the data points inside the search region decreases exponentially with the number of dimensions; e.g., for four dimensions, only about $10 \%$ are inside the search region. The large number of data points inside the hypercube but outside the search region represent wasted effort for a nearest neighbor search. In order to alleviate this effect, spatial index structures that use hyperspheres as node regions [55] have been proposed for use in nearest neighbor applications for higher dimensions. However, since this can lead to a much higher level of overlap between nodes than using hyperrectangles, a compromise is to use shapes formed by intersections of hyperspheres and hyperrectangles [35], essentially smoothing out the corners of the hyperrectangles.

In Section 4.6 we pointed out that the objects on the priority queue are contained in the leaf nodes intersected by the boundary of the search region (and similarily for the nodes on the priority queue). As the number of dimensions grows, the ratio of the number leaf nodes intersected by the boundary of the search region to the number of leaf nodes intersected by the interior of the search region tends to grow. Thus the size of the priority queue also tends to grow with the number of dimensions. For uniformly-distributed points spread evenly among the leaf nodes, where each leaf node covers about the same amount of space, it can be shown that this ratio grows exponentially with the number of dimensions. This is true even if both the search region and leaf node regions are hypercubes (i.e., if we use the Chessboard metric $L_{\infty}$ ). Of course, this is only of major significance when the number of desired neighbors is large, since the volume of the search region depends on the number of neighbors.

Some of the problems arising from operating in high-dimensional spaces can be alleviated by relaxing the requirement that the nearest neighbors be computed exactly. Our goal is to report neighbors as quickly as possible. In the incremental nearest neighbor algorithm, when an object $o$ is slightly farther from the query object $q$ than a node $n$, the algorithm must process $n$ before reporting $o$. In a high-dimensional space, as we have seen, this may cause a lot of extra work. Instead, what we can do is to report $o$ as the next nearest neighbor if its distance from $q$ is not "much" larger than that of $n$. In particular, suppose $o$ is the object on the priority queue closest to $q$, and $n$ is the node on the queue closest to $q$. We propose to report $o$ as the next (approximate) nearest neighbor if $d_{o}(q, o) \leq(1+\epsilon) d_{n}(q, n)$, where $\epsilon$ is some non-negative constant. This leads to a definition of approximate nearest neighbor that conforms to that in [4]: if $r$ is the distance of the $k^{t h}$ nearest neighbor, then the distances of the objects returned by an approximate $k$-nearest neighbor search must be no larger than $(1+\epsilon) r$. Obviously, for $\epsilon=0$ we get the exact result, and the larger $\epsilon$ is, the less exact the result is. The only change required to the incremental nearest neighbor algorithm to make it approximate in this sense is in the the key used for nodes on the priority queue. Specifically, for a node $n$ we use $(1+\epsilon) d_{n}(q, n)$ as a key instead of $d_{n}(q, n)$. In [4] $]^{5}$, it was found that a significant reduction in node accesses results from finding the $k$ approximate nearest neighbors as opposed to the $k$ exact nearest neighbors. Moreover, with relatively high probability, the result is the same in the exact and approximate cases. For example, for approximate nearest neighbor search in 16 dimensions using $\epsilon=3$ (meaning that a $300 \%$ relative error in distance is allowed), it was found [4] that improvements in running time were on the order of 10 to 50 over exact nearest neighbor search, while the average relative error was only $10 \%$ and the true nearest neighbor was found almost half the time.

\footnotetext{
${ }^{5}$ The algorithm described in [4] is not incremental, but it accesses the same set of nodes as the incremental nearest neighbor algorithm modified as described above
} 


\section{Concluding Remarks}

A detailed comparison of two approaches to browsing spatial objects in an R-tree on the basis of their distances from an arbitrary spatial query object was presented. It was shown that an incremental algorithm(INN) significantly outperforms (in terms of execution time, $\mathrm{R}$-tree node disk $\mathrm{V} / \mathrm{O}$, and object distance calculations) a solution based on a $k$-nearest neighbor algorithm $(k-\mathrm{NN})$. This was true even when the $k$-NN approach was optimized for this application by carefully choosing the increments for $k$ and using previous search results for pruning when the $k$-NN algorithm must be re-invoked. The incremental approach was also found to have superior performance when applied to the problem of computing the $k$ nearest neighbors of a given query object. Our experiments confirm that the INN algorithm achieves a higher level of pruning than the $k$-NN algorithm. This is important as it reduces the amount of $\mathrm{R}$-tree node disk $\mathrm{V} / \mathrm{O}$ as well as the number of distance calculations, which, when combined, account for a major portion of the execution time.

In the experiments reported in Section 6, we used an R-tree variant in which the spatial objects were stored directly in the leaf nodes of the R-tree. This is not always practical, especially for complex and variablesize objects such as polygons. The other alternative is to store the objects in an external file, in which case the leaf nodes store the bounding boxes of the spatial objects and pointers to the objects. We performed additional experiments where the maps used in Section 6 were stored in such an R-tree, and we used the INN variant given in Figure $4^{6}$. These experiments revealed an even larger advantage for the incremental nearest neighbor algorithm over the $k$-nearest neighbor algorithm (typically over 50\%). This is primarily because the INN algorithm accessed many fewer data objects (for the purpose of calculating their distances from the query object) than the $k$-NN algorithm. The $k$-NN algorithm typically accessed 4-6 times as many objects as the INN algorithm for low values of $k$, and up to twice as many for values of $k$ as high as $5 \%$ of the map size. Reducing the number of object accesses and object distance calculations when using the incremental algorithm has an even greater effect in terms of reducing the execution time for more complex spatial objects (e.g., polygons).

In a worst-case scenario, all the leaf nodes in the spatial data structure must be accessed (see Figure 4.8 and the discussion in Section 4.8). In contrast to the incremental algorithm presented in Figure 3, the variant presented in Figure 4 for the R-tree implementation where the spatial objects are stored external to the R-tree alleviates the worst case described above by making use of bounding rectangles in leaf nodes, thereby enabling it to avoid accessing many data objects from disk ${ }^{7}$. In particular, in the original version of the algorithm, the spatial index was not assumed to have bounding rectangles, which meant that for this worst case all data objects had to be accessed from disk in order to measure their distances from the query object. The use of bounding rectangles stored in the tree leads to a considerably more efficient (and conceptually different) incremental algorithm for R-trees in that the bounding boxes can be used as pruning devices to reduce disk I/O for accessing spatial descriptions of objects.

Future work involves comparing the behavior of the incremental nearest neighbor algorithm on different spatial data structures such as PMR quadtrees, R-trees, and $\mathrm{R}^{+}$-trees, as well as adapting the algorithm to other classes of index structures, such as distance-based indexes [11, 14, 52]. Also, we wish to investigate further the use of the algorithm with very large data sets and in high-dimensional spaces, where the priority queue may have to be stored on disk.

\footnotetext{
${ }^{6}$ We decided to report only the results of experiments where the spatial objects are stored in the leaf nodes rather than external to the R-tree. This was done, in part, because the organization of the external object storage has a large effect on the performance, and thus introduces an extra variable into the comparison of the algorithms.

${ }^{7}$ Recall from footnote 6 that we decided to report only the experiments in which the spatial objects are stored in the leaf nodes rather than external to the R-tree.
} 
To appear in ACM Transactions on Database Systems (also Univ. of MD Computer Science TR 3919)

\section{References}

[1] P. M. Aoki. Generalizing "search" in generalized search trees. In Proceedings of the 14th International Conference on Data Engineering, pages 380-389, Orlando, FL, Feb 1998.

[2] W. G. Aref and H. Samet. Uniquely reporting spatial objects: Yet another operation for comparing spatial data structures. In Proceedings of the Fifth International Symposium on Spatial Data Handling, pages 178-189, Charleston, SC, August 1992.

[3] W. G. Aref and H. Samet. Estimating selectivity factors of spatial operations. In Optimization in Databases - Fifth International Workshop on Foundations of Models and Languages for Data and Objects, pages 31-40, Aigen, Austria, September 1993. (Also Technical Report: Informatik-Bericht 93/9, Technische Univerität Clausthal, Clausthal-Zellerfeld.)

[4] S. Arya, D. M. Mount, N. S. Netanyahu, R. Silverman, and A. Wu. An optimal algorithm for approximate nearest neighbor searching. In Proceedings of the Fifth Annual ACM-SIAM Symposium on Discrete Algorithms, pages 573-582, Arlington, VA, January 1994. Revised version: http://www.cs. umd.edu/ $\sim$ mount/.

[5] L. Becker and R. H. Güting. Rule-based optimization and query processing in an extensible geometric database system. ACM Transactions on Database Systems, 17(2):247-303, June 1992.

[6] N. Beckmann, H. P. Kriegel, R. Schneider, and B. Seeger. The R*-tree: An efficient and robust access method for points and rectangles. In Proceedings of the ACM SIGMOD Conference, pages 322-331, Atlantic City, NJ, June 1990.

[7] J. L. Bentley. Multidimensional binary search trees used for associative searching. Communications of the ACM, 18(9):509-517, September 1975.

[8] S. Berchtold, C. Böhm, D. A. Keim, and H. P. Kriegel. A cost model for nearest neighbor search in high-dimensional data space. In Proceedings of the 16th ACM SIGACT-SIGMOD-SIGART Symposium on Principles of Database systems (PODS), pages 78-86, Tucson, AZ, May 1997.

[9] S. Berchtold, D. A. Keim, and H.-P. Kriegel. The X-tree: An index structure for high-dimensional data. In Proceedings of the 22nd International Conference on Very Large Data Bases, pages 28-39, Mumbai, India, September 1996.

[10] M. Bern. Approximate closest-point queries in high dimensions. Information Processing Letters, 45(2):95-99, February 1993.

[11] S. Brin. Near neighbor search in large metric space. In U. Dayal, P. M. D. Gray, and S. Nishio, editors, Proceedings of the 21st International Conference on Very Large Data Bases, pages 574-584, Zurich, Switzerland, September 1995.

[12] A. J. Broder. Strategies for efficient incremental nearest neighbor search. Pattern Recognition, 23(12):171-178, January 1990.

[13] W. A. Burkhard and R. Keller. Some approaches to best-match file searching. Communications of the $A C M, 16(4): 230-236$, April 1973. 
To appear in ACM Transactions on Database Systems (also Univ. of MD Computer Science TR 3919)

[14] P. Ciaccia, M. Patella, and P. Zezula. M-tree: An efficient access method for similarity search in metric spaces. In M. Jarke, M. J. Carey, K. R. Dittrich, F. H. Lochovsky, P. Loucopoulos, and M. A. Jeusfeld, editors, Proceedings of the 23rd International Conference on Very Large Data Bases, pages 426-435, Athens, Greece, August 1997.

[15] D. Comer. The ubiquitous B-tree. ACM Computing Surveys, 11(2):121-137, June 1979.

[16] C. M. Eastman and M. Zemankova. Partially specified nearest neighbor searches using k-d-trees. Information Processing Letters, 15(2):53-56, September 1982.

[17] C. Esperança and H. Samet. Orthogonal polygons as bounding structures in filter-refine query processing strategies. In M. Scholl and A. Voisard, editors, Advances in Spatial Databases - Fifth International Symposium, pages 197-220, Berlin, Germany, July 1997. (Also Springer-Verlag Lecture Notes in Computer Science 1262.)

[18] C. Faloutsos and K. Lin. FastMap: A fast algorithm for indexing, data-mining and visualization of traditional and multimedia datasets. In Proceedings of the ACM SIGMOD Conference, pages 163-174, San Jose, CA, May 1995.

[19] A. U. Frank and R. Barrera. The Fieldtree: A data structure for geographic information systems. In A. Buchmann, O. Günther, T. R. Smith, and Y. F. Wang, editors, Design and Implementation of Large Spatial Databases - First Symposium, pages 29-44, Santa Barbara, CA, July 1989. (Also SpringerVerlag Lecture Notes in Computer Science 409.)

[20] M. L. Fredman, R. Sedgewick, D. D. Sleator, and R. E. Tarjan. The pairing heap: A new form of selfadjusting heap. Algorithmica, 1(1):111-129, 1986.

[21] J. H. Friedman, J. L. Bentley, and R. A. Finkel. An algorithm for finding best matches in logarithmic expected time. ACM Transactions on Mathematical Software, 3(3):209-226, September 1977.

[22] K. Fukunaga and P. M. Narendra. A branch and bound algorithm for computing $k$-nearest neighbors. IEEE Transactions on Computers, 24(7):750-753, July 1975.

[23] O. Günther and H. Noltemeier. Spatial database indices for large extended objects. In Proceedings of the Seventh International Conference on Data Engineering, pages 520-526, Kobe, Japan, April 1991.

[24] A. Guttman. R-trees: A dynamic index structure for spatial searching. In Proceedings of the ACM SIGMOD Conference, pages 47-57, Boston, MA, June 1984.

[25] J. Hafner, H.S. Sawhney, W. Equitz, M. Flickner, and W. Niblack. Efficient color histogram indexing for quadratic form distance functions. IEEE Transactions on Pattern Analysis and Machine Intelligence, 17(7):729-736, July 1995.

[26] A. Henrich. A distance-scan algorithm for spatial access structures. In Proceedings of the Second ACM Workshop on Geographic Information Systems, pages 136-143, Gaithersburg, MD, December 1994.

[27] A. Henrich. The $\mathrm{LSD}^{h}$-tree: An access structure for feature vectors. In Proceedings of the 14th IEEE International Conference on Data Engineering, pages 362-369, Orlando, FL, February 1998.

[28] A. Henrich, H. W. Six, and P. Widmayer. The LSD tree: Spatial access to multidimensional point and non-point data. In P. M. G. Apers and G. Wiederhold, editors, Proceedings of the 15th International Conference on Very Large Data Bases, pages 45-53, Amsterdam, The Netherlands, August 1989. 
To appear in ACM Transactions on Database Systems (also Univ. of MD Computer Science TR 3919) 41

[29] G. R. Hjaltason and H. Samet. Ranking in spatial databases. In M. J. Egenhofer and J. R. Herring, editors, Advances in Spatial Databases - Fourth International Symposium, pages 83-95, Portland, ME, August 1995. (Also Springer-Verlag Lecture Notes in Computer Science 951.)

[30] E. G. Hoel and H. Samet. Efficient processing of spatial queries in line segment databases. In O. Günther and H. J. Schek, editors, Advances in Spatial Databases - Second Symposium, pages 237-256, Zurich, Switzerland, August 1991. (Also Springer-Verlag Lecture Notes in Computer Science 525.)

[31] I. Kamel and C. Faloutsos. On packing R-trees. In Proceedings of the Second International Conference on Information and Knowledge Management, pages 490-499, Washington, DC, November 1993.

[32] I. Kamel and C. Faloutsos. Hilbert R-tree: An improved R-tree using fractals. In J. Bocca, M. Jarke, and C. Zaniolo, editors, Proceedings of the 20th International Conference on Very Large Data Bases, pages 500-509, Santiago, Chile, September 1994.

[33] B. Kamgar-Parsi and L. N. Kanal. An improved branch and bound algorithm for computing $k$-nearest neighbors. Pattern Recognition Letters, 3(1), January 1985.

[34] K. V. R. Kanth, D. Agrawal, and A. Singh. Dimensionality reduction for similarity searching in dynamic databases. In Proceedings of the ACM SIGMOD Conference, pages 237-248, Seattle, WA, June 1998.

[35] N. Katayama and S. Satoh. The SR-tree: An index structure for high-dimensional nearest neighbor queries. In J. Peckham, editor, Proceedings of the ACM SIGMOD Conference, pages 369-380, Tucson, AZ, May 1997.

[36] F. Korn, N. Sidiropoulos, C. Faloutsos, E. Siegel, and Z. Protopapas. Fast nearest neighbor search in medical image databases. In Proceedings of the 22nd International Conference on Very Large Data Bases, pages 215-226, Mumbai, India, September 1996.

[37] H.-P. Kriegel, T. Schmidt, and T. Seidl. 3D similarity search by shape approximation. In M. Scholl and A. Voisard, editors, Advances in Spatial Databases - Fifth International Symposium, pages 11-28, Berlin, Germany, July 1997. (Also Springer-Verlag Lecture Notes in Computer Science 1262.)

[38] M. Lindenbaum and H. Samet. A probabilistic analysis of trie-based sorting of large collections of line segments. Computer Science Department TR-3455, University of Maryland, College Park, MD, April 1995.

[39] D. Lomet and B. Salzberg. A robust multi-attribute search structure. In Proceedings of the Fifth IEEE International Conference on Data Engineering, pages 296-304, Los Angeles, CA, February 1989.

[40] M. Muralikrishna and D. J. DeWitt. Equi-depth histograms for estimating selectivity factors for multidimensional queries. In Proceedings of the ACM SIGMOD Conference, pages 28-36, Chicago, IL, June 1988.

[41] O. J. Murphy and S. M. Selkow. The efficiency of using $k$ - $d$-trees for finding nearest neighbors in discrete space. Information Processing Letters, 23(4):215-218, November 1986.

[42] R. C. Nelson and H. Samet. A consistent hierarchical representation for vector data. Computer Graphics, 20(4):197-206, August 1986. (Also Proceedings of the SIGGRAPH'86 Conference, Dallas, TX, August 1986.)

[43] Bureau of the Census. Tiger/Line precensus files. Washington, DC, 1989. 
To appear in ACM Transactions on Database Systems (also Univ. of MD Computer Science TR 3919) 42

[44] J. T. Robinson. The $k-d-b$-tree: A search structure for large multidimensional dynamic indexes. In Proceedings of the ACM SIGMOD Conference, pages 10-18, Ann Arbor, MI, April 1981.

[45] N. Roussopoulos, S. Kelley, and F. Vincent. Nearest neighbor queries. In Proceedings of the ACM SIGMOD Conference, pages 71-79, San Jose, CA, May 1995.

[46] N. Roussopoulos and D. Leifker. Direct spatial search on pictorial databases using packed R-trees. In Proceedings of the ACM SIGMOD Conference, pages 17-31, Austin, TX, May 1985.

[47] H. Samet. The Design and Analysis of Spatial Data Structures. Addison-Wesley, Reading, MA, 1990.

[48] T. Seidl and H.-P. Kriegel. Optimal multi-step $k$-nearest neighbor search. In Proceedings of the ACM SIGMOD Conference, pages 154-165, Seattle, WA, June 1998.

[49] P. G. Selinger, M. M. Astrahan, D. D. Chamberlin, R. A. Lorie, and T. G. Price. Access path selection in a relational database management system. In Proceedings of the ACM SIGMOD Conference, pages 23-34, Boston, MA, June 1979.

[50] T. Sellis, N. Roussopoulos, and C. Faloutsos. The $R^{+}$-tree: a dynamic index for multi-dimensional objects. In P. M. Stocker and W. Kent, editors, Proceedings of the 13th International Conference on Very Large Databases, pages 71-79, Brighton, England, September 1987. (Also Computer Science Department, University of Maryland, College Park, MD, TR-1795.)

[51] R. F. Sproull. Refinements to nearest-neighbor searching in k-dimensional trees. Algorithmica, 6(4):579-589, 1991.

[52] J. K. Uhlmann. Satisfying general proximity/similarity queries with metric trees. Information Processing Letters, 40(4):175-179, November 1991.

[53] T. L. Wang and D. Shasha. Query processing for distance metrics. In D. McLeod, R. Sacks-Davis, and $\mathrm{H}$. Schek, editors, Proceedings of the 16th International Conference on Very Large Databases, pages 602-613, Brisbane, Australia, August 1990.

[54] D. A. White and R. Jain. Algorithms and strategies for similarity retrieval. Technical Report VCL-96-101, Visual Computing Laboratory, University of California, San Diego, CA, 1996. http://vision.ucsd.edu/papers/simret.

[55] D. A. White and R. Jain. Similarity indexing with the SS-tree. In Proceedings of the 12th IEEE International Conference on Data Engineering, pages 516-523, New Orleans, LA, February 1996. 